\title{
Sequences of stable bundles over compact complex surfaces.
}

\author{
N. P. Buchdahl \\ Department of Pure Mathematics \\ University of Adelaide \\ Adelaide, Australia 5005 \\ e-mail: nbuchdah@maths.adelaide.edu.au
}

\section{Introduction.}

In [D2], Donaldson proved that every stable holomorphic bundle on an algebraic surface equipped with a Hodge metric admits an irreducible Hermitian-Einstein connection (unique up to gauge equivalence). The fact that bundles admitting Hermitian-Einstein connections are quasi-stable (i.e., direct sums of stable bundles all of the same slope) had already been proved by Kobayashi [Kob] and Lübke [L]. Donaldson's result was subsequently generalized by a number of authors, notably Uhlenbeck-Yau [UY] who extended it to Kähler manifolds of arbitrary dimension, by Li-Yau [LY] who extended it to general manifolds equipped with Gauduchon metrics, and by the author of this paper [B3], who proved the Li-Yau result independently in complex dimension 2 by different methods.

The identification of moduli spaces of stable bundles with moduli spaces of anti-self-dual connections on an algebraic surface has led to a number of extraordinary results in the differential topology of 4-dimensional manifolds; see, e.g., [D4], [FM1], [FM2], [Kot], [OV]. A key theme in all these papers is that on an algebraic surface, the Donaldson polynomials [D5] can be expressed in terms of algebro-geometric quantities, thereby facilitating their computation. A frequently occurring problem is that the moduli spaces involved are not compact, and although there is a gauge theoretic compactification [D3] using the results of Uhlenbeck [U1], [U2], this is not ostensibly an algebraic object (but sometimes can be: see [Ma]). Instead, authors have preferred to use Gieseker's compactification [Gie] of moduli spaces of stable bundles, but this also leads to some difficulties in that Gieseker stability is not the same as Mumford-Takemoto stability [OSS] and it is the latter definition which is used in gauge theory. (Morgan [Mor] has however proved that for the purposes of computing Donaldson polynomials, the Gieseker compactification is sufficient.)

The main result (Theorems 1.3 and 1.4) proved in this paper is that sequences of stable bundles of fixed topological type and bounded degree have, after blowing-up and pulling-back sufficiently often, strongly convergent subsequences. Using this, a natural topology can be defined on the space of stable holomorphic structures of fixed degree on a given unitary bundle and its pull-backs to blowups of the surface so that (under some conditions) the space is compact - these results are given in the related paper [B5].

The results presented here give an interpretation of the theorems of Uhlenbeck in terms of the wellknown phenomenon of jumping of holomorphic structures. In addition, the proof of Theorem 1.3 has a number of other useful spin-offs: the main ones are the derivation of quite precise and explicit estimates on the curvature and connection forms of a concentrated anti-self-dual connection in a ball (anti-self-dual with respect to a flat metric), and monad descriptions of such connections. Indeed, because much of the proof is local in nature, it comes comes close to providing a compactification for moduli of anti-self-dual connections on an arbitrary smooth 4-manifold $X$ in terms of strongly converging sequences of connections on $X \# n \overline{\mathbb{C P}}_{2}$.

The author is grateful to the Isaac Newton Institute for Mathematical Sciences for its hospitality during December 1994, and to the Department of Mathematics of the University of Nantes for its hospitality during the first half of 1995 where this paper was completed.

\section{Preliminaries.}

Let $X$ be a compact complex surface and let $\omega$ be a $\bar{\partial} \partial$-closed positive $(1,1)$-form on $X$ : it is a theorem of Gauduchon [Gau] that every positive $(1,1)$-form has a unique positive conformal rescaling such the rescaled 
form is $\bar{\partial} \partial$-closed and gives the same volume $V:=\operatorname{Vol}(X, \omega):=\frac{1}{2} \int_{X} \omega^{2}$. With such a form $\omega$, the degree $\operatorname{deg}(L)=\operatorname{deg}(L, \omega)$ of a holomorphic line bundle $L$ on $X$ is unambiguously defined by the formula

$$
\operatorname{deg}(L):=\frac{i}{2 \pi} \int_{X} f_{L} \wedge \omega
$$

where $f_{L}$ is the curvature of any hermitian connection on $L$. The degree depends only on $c_{1}(L)$ if and only if $b_{1}(X)$ is even, and when this is the case $\omega$ is cohomologous modulo the image of $\partial+\bar{\partial}$ to a closed form which itself is unique up to the image of $\bar{\partial} \partial$; ([B3], Proposition 2); by the Kodaira-Enriques classification of compact complex surfaces, $b_{1}(X)$ is even iff $X$ admits a Kähler metric.

If $E$ is a holomorphic $r$-bundle on $X$, set $\operatorname{deg}(E):=\operatorname{deg}(\operatorname{det} E)$ and $\mu(E):=\operatorname{deg}(E) / r$; the latter is called the normalized degree or slope of $E$. A hermitian connection on $E$ is Hermitian-Einstein if the curvature $F$ satisfies $\widehat{F}=i \lambda \mathbf{1}$ where $\widehat{F}:=*(\omega \wedge F)=: \Lambda F, \lambda=(-2 \pi / V) \cdot \mu(E)$ and $\mathbf{1}$ is the identity endomorphism of $E$. The bundle $E$ is $($ semi-) stable if $\mu(\mathcal{S})<(\leq) \mu(E)$ for every coherent subsheaf $\mathcal{S} \subset E$ with $0<\operatorname{rank}(\mathcal{S})<r$. As mentioned in the introduction, the main result of [B3] is that a bundle admits an irreducible Hermitian-Einstein connection if and only if it is stable, this generalizing the same result proved by Donaldson [D2] in the case that $(X, \omega)$ is algebraic. A bundle admitting a Hermitian-Einstein connection is a direct sum of stable bundles all of the same slope; i.e., is quasi-stable.

If $E$ has a Hermitian-Einstein connection with curvature $F$, the equation $\omega \wedge\left(F-\frac{1}{r} \operatorname{tr} F \mathbf{1}\right)=0$ and the skew-Hermitian property of $F$ give $\operatorname{tr}\left(F-\frac{1}{r} \operatorname{tr} F \mathbf{1}\right)^{2}=\left|F-\frac{1}{r} \operatorname{tr} F \mathbf{1}\right|^{2} d V$. Since the former 4 -form is a representative for the characteristic class $8 \pi^{2}\left(c_{2}-\frac{r-1}{2 r} c_{1}^{2}\right)(E)$, this motivates defining the charge of $E, C(E)$, for an arbitrary $r$-bundle $E$ by the formula

$$
C(E):=\left(c_{2}-\frac{r-1}{2 r} c_{1}^{2}\right)(E)=\frac{1}{8 \pi^{2}} \int_{X} \operatorname{tr}\left(F-\frac{1}{r} \operatorname{tr} F \mathbf{1}\right)^{2} .
$$

The charge is non-negative for any bundle admitting a Hermitian-Einstein connection, and when this is the case, is identically zero only if the induced Hermitian-Einstein connection on the adjoint bundle is flat. Note that the charge is invariant under tensoring by line bundles: $C(E \otimes L)=C(E)$ for any such $L$. In general, $C(E \otimes A)=a C(E)+r C(A)$, where $a, r$ are the ranks of $A, E$ respectively.

As in [B3], it is often convenient to identify holomorphic bundles with integrable unitary connections on a fixed topological bundle. (If $E_{\text {top }}$ is such a bundle, a unitary connection $A$ on $E_{\text {top }}$ is integrable if the $(0,2)$-component of the curvature $F(A)$ vanishes.) The group of complex automorphisms of $E_{\text {top }}$ acts on the set of unitary connections on $E_{\text {top }}$ via

$$
(g, A) \mapsto g \cdot A \quad \text { where } \quad d_{g \cdot A}:=g \circ \bar{\partial}_{A} \circ g^{-1}+g^{*-1} \circ \partial_{A} \circ g^{*},
$$

and the corresponding curvatures are related by

$$
F^{0,2}(g \cdot A)=g F^{0,2}(A) g^{-1}, \quad F^{1,1}(g \cdot A)=g\left[F(A)+\bar{\partial}_{A}\left(h^{-1} \partial_{A} h\right)\right] g^{-1} \quad \text { for } \quad h:=g^{*} g .
$$

The action preserves the set of integrable connections, and two such connections induce isomorphic holomorphic structures iff they lie in the same orbit.

The metrics on blowups of $X$ which will be used to define stability are as described in [B3], recalled here briefly for convenience. For more details, see $[\mathrm{B} 5, \S 3]$. In the discussion which follows, it is not assumed that $X$ is compact.

Let $\tilde{X} \stackrel{\pi}{\rightarrow} X$ be the blowup of $X$ at $x_{0} \in X$. Let $L_{0}:=\pi^{-1}\left(x_{0}\right)$ be the exceptional divisor so $\pi^{*} \omega$ is everywhere non-negative and is degenerate only in directions tangent to $L_{0}$. Let $\sigma$ be $i / 2 \pi$ times the curvature form of any hermitian connection on the line bundle $\mathcal{O}\left(-L_{0}\right)=: \mathcal{O}(1)$ restricting positively to $L_{0}$, and let $\omega_{\epsilon}:=\pi^{*} \omega+\epsilon \sigma$ for $\epsilon>0$; (recall $\left.\mathcal{O}\left(L_{0}\right)\right|_{L_{0}} \simeq \mathcal{O}_{L_{0}}(-1)$ ). It follows that if $\epsilon$ is sufficiently small then $\omega_{\epsilon}$ defines a positive form in a neighbourhood of $L$; if $\sigma$ is compactly supported in $\widetilde{X}$ then $\omega_{\epsilon}$ is everywhere positive for sufficiently small $\epsilon$. Note that $\sigma$ can be taken to have support in neighbourhoods of $L_{0}$ of the form $\pi^{-1}(U)$ where $U$ is an arbitrarily small neighbourhood of $x_{0}$. If $\omega$ is $\bar{\partial} \partial$-closed and $\sigma$ is compactly supported, it follows from the fact that $L$ has self-intersection -1 that $\operatorname{Vol}\left(\widetilde{X}, \omega_{\epsilon}\right)=\operatorname{Vol}(X, \omega)-\epsilon^{2} / 2$, and if $\omega$ is $d$-closed, then so too is $\omega_{\epsilon}$.

Now let $\widetilde{X} \stackrel{\pi}{\rightarrow} X$ be a modification of $X$ consisting of $n$ blowups, and let $\sigma_{i}$ be a closed smooth $(1,1)$ form on $\widetilde{X}$ corresponding as in the last paragraph to the $i$-th blowup (pulled-back to $\widetilde{X}$ ). Let $\mathbb{R}_{+}^{n}:=\{\alpha=$ 
$\left.\left(\alpha_{1}, \ldots, \alpha_{n}\right) \in \mathbb{R}^{n} \mid \alpha_{i}>0, i=1, \ldots, n\right\}$ and for $\alpha \in \mathbb{R}_{+}^{n}$ let $\rho_{\alpha}:=\sum \alpha_{i} \sigma_{i}$, so $\rho_{\alpha} \cdot \rho_{\alpha}=-\sum \alpha_{i}^{2}=:-|\alpha|^{2}$; (this definition differs slightly from that in [B3] where $\rho$ has the opposite sign). The vector $\alpha \in \mathbb{R}_{+}^{n}$ will be called suitable if $\omega_{\alpha}:=\pi^{*} \omega+\rho_{\alpha}$ defines a positive $\bar{\partial} \partial$-closed $(1,1)$-form on $\widetilde{X}$ for some choice of the forms $\sigma_{i}$. If $\widetilde{X}^{\prime}$ is another modification of $X$ consisting of $n$ blowups, each $\sigma_{i}$ on $\widetilde{X}$ corresponds to a $\sigma_{i}^{\prime}$ on $\widetilde{X}^{\prime}$ in $H^{2}(\widetilde{X}, \mathbb{R})$ since $\widetilde{X}$ and $\widetilde{X}^{\prime}$ are diffeomorphic so the degrees of line bundles on $\widetilde{X}$ and $\widetilde{X}^{\prime}$ defined by $\pi^{*} \omega+\rho_{\alpha}$ and $\pi^{\prime *} \omega+\rho_{\alpha}^{\prime}$ are the same. The notation will sometimes be abused by using the one symbol $\omega_{\alpha}$ to denote a metric on a blowup, even though the blowup may be changing.

The following result summarises some of the relationships between stability on $X$ and $\widetilde{X}$; the proof is given in $[\mathrm{B} 5, \S 3]$ :

\section{Proposition 1.2.}

(a) If $\widetilde{E}$ is a bundle on $\widetilde{X}$ such that $\pi_{*} \widetilde{E}$ is stable, then there exists $\epsilon>0$ such that $\widetilde{E}$ is $\omega_{\alpha}$-stable for all suitable $\alpha \in \mathbb{R}_{+}^{n}$ satisfying $|\alpha|<\epsilon$;

(b) If $\widetilde{E}$ on $\widetilde{X}$ is $\omega_{\epsilon \alpha}$-semi-stable for all $\epsilon>0$ sufficiently small, then $\pi_{*} \widetilde{E}$ is semi-stable;

(c) If $b_{1}(X)$ is even, then for any $r_{0}, C_{0}$ there exists $\epsilon_{0}=\epsilon_{0}\left(\omega, r_{0}, C_{0}\right)>0$ with the property that any bundle $\widetilde{E}$ on $\widetilde{X}$ of rank $\leq r_{0}$ and charge $\leq C_{0}$ which is $\omega_{\alpha}$-stable for some suitable $\alpha \in \mathbb{R}_{+}^{n}$ satisfying $|\alpha|<\epsilon_{0}$ is also $\omega_{\epsilon \alpha}$-stable for all $\epsilon \in\left(0, \epsilon_{0} /|\alpha|\right)$.

In particular, the pull-backs of stable bundles are stable, and when $b_{1}(X)$ is even, the pull-backs of stable bundles all of the same topological type are stable with respect to the same metric on the blowup.

Note that for any bundle $\widetilde{E}$ on $\widetilde{X}$, the direct image $\pi_{*} \widetilde{E}$ is a torsion-free sheaf on $X$. The double-dual $\left(\pi_{*} \widetilde{E}\right)^{* *}$ is reflexive, hence locally free [OSS], and a torsion-free sheaf is (semi-)stable iff its double-dual is. It is not hard to show that $C\left(\left(\pi_{*} \widetilde{E}\right)^{* *}\right) \leq C(\widetilde{E})$ with equality iff $\widetilde{E}=\left(\pi^{*} E\right) \otimes L$ for some bundle $E$ on $X$ and some line bundle $L$ on $\widetilde{X}$ trivial off the exceptional divisor; ([B5, Proposition 2.9]).

Let $\left\{E_{i}\right\}$ be a sequence of stable bundles on $(X, \omega)$ all of the same topological type and of uniformly bounded degree. Then by Uhlenbeck's weak compactness theorem [U1], [S], there is a finite set $S \subset X$ such that, after gauge transformations, a subsequence of the corresponding sequence of Hermitian-Einstein connections converges weakly in $L_{2}^{p}$ for some fixed $p>4$ and strongly in $C^{1}$ on compact subsets of $X \backslash S$ to a Hermitian-Einstein connection which, by removability of singularities [U2], then extends across $S$ to define a Hermitian-Einstein connection on a bundle $E$ with $C(E) \leq C\left(E_{i}\right)$, endowing $E$ with the structure of a quasi-stable holomorphic bundle on $(X, \omega)$. This type of convergence will be summarised by saying that the subsequence of $\left\{E_{i}\right\}$ converges weakly to $E$ (off $S$ ) (with respect to $\omega$ ). If $S$ is empty, the convergence will be called "strong convergence" (with respect to $\omega$ ).

Suppose for the moment that $b_{1}(X)$ is even and that the weak limit $E$ is stable. Blow up $X$ along $S$ to $\widetilde{X}$ and fix a metric on $\widetilde{X}$ of the form $\omega_{\alpha}$, where $|\alpha|<\epsilon_{0}$ as in Proposition 1.2(c). Then $\pi^{*} E_{i}$ is $\omega_{\alpha}$-stable for all $i$ so by weak compactness on $\left(\widetilde{X}, \omega_{\alpha}\right)$, there is a finite set $\widetilde{S} \subset \widetilde{X}$ such that a subsequence of $\left\{\pi^{*} E_{i}\right\}$ converges weakly to some $\omega_{\alpha}$-quasi-stable bundle $\widetilde{E}$ on $\widetilde{X}$. Using Lemmas 2.1 and 2.2 of the next section, it is not hard to show that under these circumstances, $\widetilde{S} \subset \pi^{-1}(S), \widetilde{E}$ is stable, and $\left(\pi_{*} \widetilde{E}\right)^{* *}=E$.

If it could be shown that $\widetilde{E}$ were non-trivial on the exceptional divisor, then the amount of charge bubbled by the sequence on the blowup would be strictly less than that bubbled on $X$, and therefore by induction on this amount, iteration of the procedure would eventually lead to a strongly convergent sequence on some blowup. Unfortunately, there is no guarantee that the amount bubbled on the blowup is not the same as that bubbled on the original surface, and indeed examples can be constructed where this occurs. However, if instead of blowing up $X$ along the fixed set $S$, the points in the center of the modification are allowed to vary (but converge to $S$ ), then strongly convergent subsequences can be obtained:

Theorem 1.3. Let $X$ be a compact complex surface equipped with a positive $\bar{\partial} \partial$-closed $(1,1)$-form $\omega$. Let $\left\{A_{i}\right\}$ be a sequence of Hermitian-Einstein connections on a fixed unitary bundle $E_{\text {top }}$ of rank $r$ over $X$ such that the corresponding holomorphic bundles $E_{i}$ are stable and are of uniformly bounded degree. Suppose that $E_{i}$ converges weakly to $E$ off $S \subset X$. Then there is a subsequence $\left\{E_{i_{j}}\right\} \subset\left\{E_{i}\right\}$ such that:

1. There is a sequence of blowups $\widetilde{X}_{i_{j}}$ of $X$ consisting of $n \leq 2 C\left(E_{\mathrm{top}}\right)-2 C(E)-1$ blowups at simple points converging to a blowup $\widetilde{X} \stackrel{\pi}{\rightarrow} X$ of $X$;

2. The exceptional divisor of $\widetilde{X}$ is $\pi^{-1}(S)$; 
3. There are complex automorphisms $g_{i_{j}}$ of $\pi_{i_{j}}^{*} E_{\text {top }}$ such that $\left\{g_{i_{j}} \cdot\left(\pi_{i_{j}}^{*} A_{i_{j}}\right)\right\}$ converges strongly in $C^{1}$ to define a holomorphic bundle $\widetilde{E}$ on $\widetilde{X}$ with $\left(\pi_{*} \widetilde{E}\right)^{* *}=E$;

4. For each compact set $K \subset X \backslash S$ there is a constant $C_{K}$ such that $\sup _{K}\left(\left|g_{i_{j}}\right|+\left|g_{i_{j}}^{-1}\right|\right) \leq C_{K}$ for all $i_{j}$, with $\left\{g_{i_{j}}\right\}$ converging uniformly in $C^{2}(K)$;

5. For any choice $T$ of $r^{2}+1$ disjoint balls in $X \backslash S$ and sufficiently small suitable $\alpha \in \mathbb{R}_{+}^{n}$ the connections $g_{i_{j}} \cdot\left(\pi_{i_{j}}^{*} A_{i_{j}}\right)$ can be taken to be Hermitian-Einstein with respect to $\pi_{i_{j}}^{*} \omega+\rho_{\alpha}$ on the complement of $T$;

6. If $E$ is stable, then for any suitable $\alpha \in \mathbb{R}_{+}^{n}$ sufficiently small, the bundles $\pi_{i_{j}}^{*} E_{i_{j}}$ are $\left(\pi_{i_{j}}^{*} \omega+\rho_{\alpha}\right)$-stable, $\widetilde{E}$ is $\omega_{\alpha}$-stable, and the connections $g_{i_{j}} \cdot\left(\pi_{i_{j}}^{*} A_{i_{j}}\right)$ can be taken to be Hermitian-Einstein with respect to $\pi_{i_{j}}^{*} \omega+\rho_{\alpha}$.

The convergence of a sequence of blowups should be interpreted as the convergence of a sequence of integrable complex structures on the same underlying smooth manifold $X \# n \overline{\mathbb{P}}_{2}$ endowed with a fixed Riemannian metric. In the case in hand, these complex structures are isomorphic on the complement of a fixed open set with strictly pseudoconvex boundary.

It is evident from the statement of the theorem that complications arise when the weak limit $E$ is only semi-stable but not stable. However, for bundles of rank 2, these difficulties can be overcome:

Theorem 1.4. Under the hypotheses of Theorem 1.3, suppose in addition that $b_{1}(X)$ is even and that $E_{\text {top }}$ has rank 2 . Then there is a subsequence $\left\{E_{i_{j}}\right\} \subset\left\{E_{i}\right\}$ such that:

1. There is a sequence of blowups $\widetilde{X}_{i_{j}}$ of $X$ consisting of $n \leq 2 C\left(E_{\mathrm{top}}\right)-1$ blowups at simple points converging to a blowup $\widetilde{X} \stackrel{\pi}{\rightarrow} X$ of $X$;

2. For some suitable $\alpha \in \mathbb{R}_{+}^{n}, \pi_{i_{j}}^{*} E_{i_{j}}$ is $\left(\pi_{i_{j}}^{*} \omega+\rho_{\alpha}\right)$-stable and the corresponding sequence of HermitianEinstein connections converges strongly on $\widetilde{X}$ to define an $\omega_{\alpha}$-stable bundle $\widetilde{E}$ on $\widetilde{X}$;

3. $\operatorname{det} \widetilde{E}=\pi^{*} \operatorname{det} E, \pi_{*} \widetilde{E}$ is semi-stable, and there are non-zero homomorphisms $\left(\pi_{*} \widetilde{E}\right)^{* *} \rightarrow E, E \rightarrow$ $\left(\pi_{*} \widetilde{E}\right)^{* *}$.

Note that the intertwining operators $g_{i_{j}}$ linking the two sequences of connections can no longer be guaranteed to enjoy the same uniform bounds as given by the fourth statement in Theorem 1.3.

Let $E_{\text {top }}$ be a unitary $r$-bundle over $(X, \omega)$ and let $\mathcal{M}\left(X, E_{\text {top }}\right)$ denote the space of isomorphism classes of quasi-stable holomorphic structures on $E_{\text {top }}$. Consider the set of pairs $(\widetilde{X}, \widetilde{E})$ where

1. $\tilde{X}$ is a blowup of $X$;

2. $\quad \widetilde{E}$ is a holomorphic bundle on $\widetilde{X}$ topologically isormorphic to $\pi^{*} E_{\text {top }}$ such that $\pi_{*} E$ is semi-stable;

3. $\widetilde{E}$ is $\omega_{\alpha}$-quasi-stable for all suitable $\alpha$ in an open set of such;

4. If $b_{1}(X)$ is odd, $\operatorname{deg}\left(\pi_{*} \widetilde{E}, \omega\right)=0$.

On this set, define an equivalence relation $\sim$ by setting $(\widetilde{X}, \widetilde{E}) \sim\left(X^{\prime}, E^{\prime}\right)$ iff there is a joint blowup $\widetilde{X}^{\prime}$ such that the pull-backs of $\widetilde{E}$ and $E^{\prime}$ to $\widetilde{X}^{\prime}$ are isomorphic in the usual sense, and let $\overline{\mathcal{M}}\left(X, E_{\text {top }}\right)$ denote the set of equivalence classes.

A topology is defined on $\overline{\mathcal{M}}$ by defining $\left\{\left[\left(\widetilde{X}_{i}, \widetilde{E}_{i}\right)\right]\right\} \subset \overline{\mathcal{M}}$ to converge to $[(\widetilde{X}, \widetilde{E})]$ iff $\left[\left(\widetilde{X}_{i}, \widetilde{E}_{i}\right)\right]$ can be represented by a sequence of blowups $\widetilde{X}_{i}$ converging to $\widetilde{X}$ with $\omega_{\alpha}$-stable bundles $\widetilde{E}_{i}$ on $\widetilde{X}_{i}$ converging strongly to $\widetilde{E}$ on $\widetilde{X}$.

The subset $\mathcal{M}\left(X, E_{\text {top }}\right) \subset \overline{\mathcal{M}}\left(X, E_{\text {top }}\right)$ consists of the bundles on blowups which are holomorphically trivial on the exceptional divisor. This is an open set for if $\left\{\widetilde{E}_{i}\right\} \subset \overline{\mathcal{M}} \backslash \mathcal{M}$ converges to $\widetilde{E}$ then each $\widetilde{E}_{i}$ in a subsequence is non-trivial on some irreducible component $L$ of the exceptional divisor and by semi-continuity of cohomology, the fact that $H^{0}\left(L, \widetilde{E}_{i_{j}}(-1)\right) \neq 0$ implies $H^{0}(L, \widetilde{E}(-1)) \neq 0$. Theorems 1.3 and 1.4 imply that under certain restrictions, every sequence in $\mathcal{M}$ has a subsequence converging in $\overline{\mathcal{M}}$.

In $[\mathrm{B} 5, \S 6]$ it is proved that if $b_{1}(X)$ is even and $\operatorname{rank}\left(E_{\mathrm{top}}\right)=2$ then $\overline{\mathcal{M}}\left(X, E_{\mathrm{top}}\right)$ is compact. It is also proved that if every bundle $E$ on $X$ with $\operatorname{rank}(E)=\operatorname{rank}\left(E_{\mathrm{top}}\right), c_{1}(E)=c_{1}\left(E_{\mathrm{top}}\right)$ and $C(E) \leq C\left(E_{\mathrm{top}}\right)$ which is semi-stable is actually stable, then $\overline{\mathcal{M}}\left(X, E_{\text {top }}\right)$ is compact and Hausdorff space. If $b_{1}(X)$ is even and $c_{1}\left(E_{\text {top }}\right)$ is not a torsion class in $H^{2}\left(X, \mathbb{Z}_{r}\right)$ for $r=\operatorname{rank}\left(E_{\text {top }}\right)$, this condition will be satisfied for generic $\omega$. If $b_{1}(X)$ is odd, it will only be satisfied if $c_{2}\left(E_{\text {top }}\right)<0$ (since $\left(L^{*} \otimes \operatorname{det} E\right) \oplus \mathcal{O}^{r-1}$ is semi-stable if $c_{1}(L)=0$ and $\operatorname{deg}(L)=\operatorname{deg}(E) / r$; the existence of such $L$ follows from the proof of Proposition 2 in [B3]).

To outline the structure of the remainder of this paper, let $(X, \omega)$ be a given compact hermitian surface with $\bar{\partial} \partial \omega=0$, and let $\left\{A_{i}\right\}$ be a sequence of Hermitian-Einstein connections on a unitary $r$-bundle $E_{\text {top }}$ over $X$ which is converging in $C^{1}$ on compact subsets of the complement of some non-empty finite set $S \subset X$. 
Suppose $x_{0} \in S$. After twisting $A_{i}$ by a converging sequence of Hermitian-Einstein connections on a trivial line bundle in a neighbourhood of $x_{0}$, it can be assumed without loss of generality that $A_{i}$ is anti-selfdual in this neighbourhood. Assume for the moment that the metric $\omega$ is the standard flat metric in this neighbourhood, and dilate to the ball $B\left(x_{0}, 5\right)$ of radius 5 . Transferring everything to $\mathbb{C}^{2}$ gives a sequence of anti-self-dual connections $A_{i}$ on an $S U(r)$ bundle over the ball which are converging in $C^{1}$ on compact subsets of $B(0,5) \backslash\{0\}$. By dilating further if necessary, it can be assumed that the limiting connection $A$, after removal of singularities, satisfies $\|F(A)\|_{L^{2}}^{2} \leq \epsilon$ where $\epsilon>0$ can be prescribed arbitrarily small, and $\lim ||\left|F\left(A_{i}\right) \|^{2}-8 \pi^{2} k\right| \leq \epsilon$ for some integer $k>0$.

In $\S 2$, it will be shown that (perhaps after further dilations) the connections $A_{i}$ can be truncated by a fixed cutoff function $\rho$ and the resulting connections $\rho A_{i}$ can be perturbed to anti-self-dual connections $\rho A_{i}+a_{i}$ on a bundle over $S^{4}$ with $c_{2}=k$ (i.e., instantons). Such connections have an explicit description in terms of monads on $\mathbb{P}_{2}$, that is, in terms of linear algebraic data ([D1]), and the connections $\rho A_{i}$ can be estimated by explicit calculation of the instanton connections; this is performed in $\S \S 3-4$.

Although $\rho A_{i}+a_{i}$ provides a good approximation to $\rho A_{i}$, it is unfortunately not the case that after pulling back to the blowup of $\mathbb{C}^{2}$ at the origin, the Hermitian-Einstein connection corresponding to $\pi^{*}\left(\rho A_{i}+\right.$ $\left.a_{i}\right)$ necessarily provides a good approximatio to that for $\pi^{*}\left(\rho A_{i}\right)$ : the reason for this is that although bounded functions pull back to bounded functions on the blowup, $L^{2}$ functions do not pull-back to $L^{2}$ functions. Thus in $\S 5$ a further perturbation is required to obtain a sequence of instantons on $S^{4}$ which does have this good approximation property, and this then reduces the convergence problem for $\left\{\rho A_{i}\right\}$ to that of a sequence of genuine instantons on $\mathbb{C}^{2}$.

In $\S 6$ it is shown that the main compactness result holds for sequences of instantons on $S^{4}$ viewed as holomorphic bundles on $\mathbb{P}_{2}$ trivial on the line at infinity, with the condition of stability suppressed by fixing trivialisations on this line - this is a purely algebraic calculation involving monads on $\mathbb{P}_{2}$ and a blowup at a point. Following this, the last pieces of the proof of Theorem 1.3 are put in place. The last section is devoted to the proof of Theorem 1.4, which consists largely of sheaf theory.

\section{Transferring concentrated connections.}

Let $X$ be a compact complex surface, $\omega$ be a $\bar{\partial} \partial$-closed positive $(1,1)$-form on $X$, and let $\left\{A_{i}\right\}$ be a sequence of irreducible Hermitian-Einstein connections on a unitary bundle over $X$ which are converging in $C^{1}$ on compact subsets of $X \backslash S$ for some finite set $S \subset X$.

In $\S 1$, it was assumed that the metric $\omega$ was flat in a neighbourhood of each of the points of $S$. To see that this is possible, let $x_{0}$ be a point of $S$ and choose holomorphic coordinates $z=\left(z^{0}, z^{1}\right)$ in a neighbourhood of $x_{0}$ such that $\omega$ agrees with the standard metric $\omega_{0}=(i / 2) \partial \bar{\partial}|z|^{2}$ at $x_{0}$. Since $\omega$ is $\bar{\partial} \partial$-closed, there is a $(1,0)$-form $u$ in a neighbourhood of $x_{0}$ such that $\omega=\omega_{0}+\bar{\partial} u+\partial \bar{u}$ in that neighbourhood, and a simple Taylor expansion argument shows that $u$ can be chosen to vanish to second order at $x_{0}$. Then if $\varphi:[0, \infty) \rightarrow[0,1]$ is a smooth function which is 0 on $\left[0, r_{1}\right]$ and 1 on $\left[2 r_{1}, \infty\right)$, for sufficiently small $r_{1}>0$ the form $\omega^{\prime}:=\omega_{0}+\bar{\partial}\left[\varphi\left(|z|^{2}\right) u\right]+\partial\left[\varphi\left(|z|^{2}\right) \bar{u}\right]$ defines a $(1,1)$-form on $X$ which is everywhere positive, is $\bar{\partial} \partial$-closed, agrees with the standard metric in a neighbourhood of $x_{0}$ and with $\omega$ on the complement of a slightly larger neighbourhood, and is homologous to $\omega$ modulo the image of $\partial+\bar{\partial}$. Hence the notions of (semi-)stability for the two metrics coincide.

The following result shows that under certain circumstances, the notions of weak convergence for the two metrics also coincide - in this lemma, it is not necessary to assume that $X$ is compact:

Lemma 2.1. Let $\omega, \omega^{\prime}$ be positive $(1,1)$-forms on $X$. Let $\left\{A_{i}\right\},\left\{A_{i}^{\prime}\right\}$ be sequence of unitary connections on a bundle such that $A_{i}^{\prime}=g_{i} \cdot A_{i}$ for some complex automorphism, and suppose that the following conditions hold:

1. $\left\|F\left(A_{i}\right)\right\|_{L^{2}}+\left\|F\left(A_{i}^{\prime}\right)\right\|_{L^{2}}$ is uniformly bounded;

2. $\left\|\omega \wedge F\left(A_{i}\right)\right\|_{L^{p}}+\left\|F^{0,2}\left(A_{i}\right)\right\|_{L^{p}}+\left\|\omega^{\prime} \wedge F\left(A_{i}^{\prime}\right)\right\|_{L^{p}}+\left\|F^{0,2}\left(A_{i}^{\prime}\right)\right\|_{L^{p}}$ is uniformly bounded for some $p>4$;

3. There are finite subsets $S, S^{\prime} \subset X$ such that $\left\{A_{i}\right\}$ converges in $C^{1}$ on compact subsets of $X \backslash S$, and $\left\{A_{i}^{\prime}\right\}$ converges in $C^{1}$ on compact subsets of $X \backslash S^{\prime}$;

4. After gauge transformations, the limits $A_{\infty}, A_{\infty}^{\prime}$ extend to connections on some other unitary bundles over $X$.

If $\sup _{Q}\left(\left|g_{i}\right|+\left|g_{i}^{-1}\right|\right)$ is uniformly bounded for some $Q$ which is the complement of a union of non-intersecting closed balls containing the points of $S \cup S^{\prime}$ then 
1. $S=S^{\prime}$

2. a subsequence of $\left\{g_{i}\right\}$ converges in $C^{2}$ on compact subsets of $X \backslash S$ to some $g_{\infty}$;

3. there are unitary automorphisms $u, u^{\prime}$ such that $u g_{\infty} u^{\prime-1}$ extends to a $C^{2}$ isomorphism of bundles over $X$;

4. for any $\epsilon>0$ there exists $r>0$ such that $\left|\int_{B\left(x_{0}, r\right)}\left(\left|F\left(A_{i}\right)\right|^{2}-\left|F\left(A_{i}^{\prime}\right)\right|^{2}\right) d V\right| \leq \epsilon$ for all $i$ and every $x_{0} \in S$

5. if $\omega=\omega^{\prime}$ then $\sup _{X}\left(\left|g_{i}\right|+\left|g_{i}^{-1}\right|\right)$ is uniformly bounded.

Proof: If $x_{0} \in S^{\prime} \backslash S$, then in some small ball around $x_{0}$ the sequence $\left\{A_{i}\right\}$ is converging. The uniform $L^{p}$ bound on $\omega^{\prime} \wedge F\left(g_{i} \cdot A_{i}\right)$ together with the Maximum Principle and the bound on $\left|g_{i}\right|+\left|g_{i}^{-1}\right|$ on the boundary of this ball then give a uniform $C^{0}$ bound on $\left|g_{i}\right|+\left|g_{i}^{-1}\right|$ over this ball. By Lemma 2.1 of [B4] the hypotheses imply that $x_{0}$ is not in fact a bad point for the sequence $\left\{A_{i}^{\prime}\right\}$ so $S^{\prime} \subset S$, and by symmetry it follows $S=S^{\prime}$.

The uniform bounds on $g_{i}$ and $g_{i}^{-1}$ together with the convergence of the sequence of connections implies that a subsequence of $\left\{g_{i}\right\}$ converges in $C^{2}$ on compact subsets of $X \backslash S$ and the existence of $u, u^{\prime}$ follows from the existence the gauge transformations enabling the limiting connections to extend, together with Hartogs' Theorem.

To prove the fourth statement, choose $r$ so small that $\left|\int_{B\left(x_{0}, r\right)}\left(\left|F\left(A_{\infty}\right)\right|^{2}+\left|F\left(A_{\infty}^{\prime}\right)\right|^{2}\right) d V\right| \leq \epsilon / 3$. By Stokes' Theorem and the fact that on the boundary of $B\left(x_{0}, r\right)$ the connections are converging in $C^{1}$ it follows that $\left|\int_{B\left(x_{0}, r\right)}\left(\operatorname{tr} F\left(A_{i}\right)^{2}-\operatorname{tr} F\left(A_{i}^{\prime}\right)^{2}\right)\right| \leq \epsilon / 2$ for $i$ sufficiently large. With respect to $\omega$,

$$
\begin{aligned}
\left\|F\left(A_{i}\right)\right\|_{L^{2}\left(B\left(x_{0}, r\right)\right)}^{2} & =\int_{B\left(x_{0}, r\right)} \operatorname{tr} F\left(A_{i}\right)^{2}+2\left\|F_{+}\left(A_{i}\right)\right\|_{L^{2}\left(B\left(x_{0}, r\right)\right)}^{2} \\
& \leq \int_{B\left(x_{0}, r\right)} \operatorname{tr} F\left(A_{i}\right)^{2}+\text { Const. } r^{4(p-2) / p}\left\|F_{+}\left(A_{i}\right)\right\|_{L^{p}\left(B\left(x_{0}, r\right)\right)}^{2},
\end{aligned}
$$

and because of the uniform bound on the $L^{p}$ norm of $F_{+}\left(A_{i}\right)$, the second term can be made arbitrarily small by choosing $r$ small enough. The same argument applies to the other sequence using $\omega^{\prime}$, and the result follows since the two metrics compare uniformly on $X$.

The last statement follows easily from the Maximum Principle, the local existence of uniformly bounded solutions to $\triangle u=\left|\widehat{F}\left(A_{i}\right)\right|$, the Sobolev Embedding theorem and Theorem 9.20 of [GT].

To find intertwining operators $g_{i}$ satisfying the hypotheses of Lemma 2.1 usually involves stability of either of the two limits, together with the following version of semi-continuity of cohomology:

Lemma 2.2. Let $Q$ be an open subset of $X$ with (possibly empty) smooth strictly pseudo-concave boundary. Let $\left\{A_{i}\right\}$ be a sequence of integrable connections on a unitary bundle converging weakly in $L_{2}^{p}$ and uniformly in $C^{1}$ on a neighbourhood of $\bar{Q}$ to $A_{\infty}$. If $s_{i}$ are non-zero sections over $Q$ satisfying $\bar{\partial}_{i} s_{i}=0$ and $\left\|s_{i}\right\|_{L^{2}(Q)}=1$, then there exists a subsequence converging in $C^{2}$ on a neighbourhood of $\bar{Q}$ to a section $s_{\infty}$ satisfying $\bar{\partial}_{\infty} s_{\infty}=0$ and $\left\|s_{\infty}\right\|_{L^{2}(Q)}=1$.

Proof: On any compact set $K \subset Q$, ellipticity of the $\bar{\partial}$-operator and convergence of the sequence of connections gives a uniform $C^{0}(K)$ bound on $s_{i}$ in terms of an $L^{2}(Q)$ bound. In a sufficiently small neighbourhood of any $x_{0} \in \partial Q$ the sequence of sections can be viewed as a sequence of vector-valued holomorphic functions over this neighbourhood. Strict pseudoconvexity of $\partial(X \backslash Q)$ and the fact that $\operatorname{dim}(X)>1$ imply that these functions extend across the boundary, and Cauchy estimates give $C^{0}$ bounds on the extensions in terms of $C^{0}$ bounds on a compact subset of $Q$. Hence there is an a priori $C^{0}$ bound on the sections $s_{i}$ over a fixed neighbourhood of $\bar{Q}$. Ellipticity of $\bar{\partial}$ and convergence of the sequence of connections then gives a uniform $L_{3}^{p}$ bound on $s_{i}$ over a slightly smaller neighbourhood and hence there is a subsequence $\left\{s_{i_{j}}\right\}$ converging strongly in $C^{2}(\bar{Q})$ using compactness of the embedding $L_{3}^{p} \subset C^{2}$ for $p>4$.

Applying these results to the sequence $\left\{A_{i}\right\}$, let $E_{i}$ be the holomorphic bundle defined by $A_{i}$ and suppose that the weak limit $E$ defined by the sequence is also stable. Since each $E_{i}$ is also stable with respect to the flattened metric $\omega^{\prime}$, there is another finite set $S^{\prime} \subset X$ such that the sequence of $\omega^{\prime}$-Hermitian-Einstein connections $\left\{A_{i}^{\prime}\right\}$ has a subsequence converging weakly off $S^{\prime}$ to define an $\omega^{\prime}$-quasi-stable bundle $E^{\prime}$. Choose a disjoint union of balls surrounding the points of $S \cup S^{\prime}$ and let $Q$ be the complement. If $g_{i}$ is an intertwining operator (uniquely determined up to scale) such that $A_{i}^{\prime}=g_{i} \cdot A_{i}$, renormalise $g_{i}$ so that $\left\|g_{i}\right\|_{L^{2}(Q)}=1$. By Lemma 2.2 a subsequence converges in $C^{2}$ to a non-zero limit $g_{\infty}$ which then extends to the whole of $X$ by Hartogs' Theorem. Since $E$ is stable and $E^{\prime}$ is semi-stable (with respect to either metric), $g_{\infty}$ is an 
isomorphism, and therefore Lemma 2.1 now applies to show that $S=S^{\prime}$ and that the amount of charge bubbled by each sequence is the same at each point of $S$.

Let $A$ be a typical connection in the sequence $\left\{A_{i}\right\}$ (where it is now assumed that the metric $\omega$ is standard in a neighbourhood of each point of $S$ ). After twisting the connection by a fixed Hermitian-Einstein connection on a trivial line bundle in a neighbourhood of $x_{0}$, it can be assumed without loss of generality that $A$ is anti-self-dual; (if $b_{1}(X)$ is odd, it should be assumed from the outside that every connection in the sequence has degree 0). Fix a small number $\eta>0$ and choose $r>0$ sufficiently small that the $L^{2}$ norm of $F(A)$ over the annulus $B\left(x_{0}, 2 r\right) \backslash B\left(x_{0}, r\right)$ is less than $\eta$. If $\eta$ is sufficiently small, it follows as in Chapter 9 of [FU] that after pulling back the connection from $B\left(x_{0}, 4 r\right)$ to $B\left(x_{0}, 4\right)$ there is a gauge transformation over the annulus $B\left(x_{0}, 4\right) \backslash B\left(x_{0}, 3\right)$ so that the gauged connection, also denoted by $A$, has its $C^{1}$ norm over the annulus bounded by a constant (independent of $A$ ) times $\eta$. Using a cutoff function $\rho$, the connection form can be cut off to define a connection $\rho A$ on $\mathbb{C}^{2}$ which is trivial outside $B(0,4)$, anti-self-dual (with respect to the standard metric) on a neighbourhood of $\bar{B}(0,3)$, and has self-dual curvature everywhere bounded in $C^{0}$ by a term of order $\eta$; bounds of the same order hold if the flat metric is replaced by the standard conformally flat metric on $S^{4}$. The following result shows that $\rho A$ can be perturbed into an anti-self-dual connection on $S^{4}$ :

Lemma 2.3. Let $Y$ be a compact Riemannian 4-manifold with anti-self-dual Weyl curvature and positive scalar curvature and let $E$ be a unitary bundle over $Y$. Then there are constants $\delta, C>0$ such that if $A$ is a smooth connection on $E$ satisfying sup $\left|F_{+}(A)\right| \leq \delta$ it follows that there is a solution $a \in \Lambda^{1}\left(\operatorname{End}_{0} E\right)$ to the equation $F_{+}(A+a)=0$ satisfying

$$
\|a\|_{L^{4}}+\left\|d_{0} a\right\|_{L^{2}} \leq C \sup \left|F_{+}(A)\right| .
$$

( $d_{0} a$ is the full covariant derivative of a using the connection A.)

Proof: The argument is modeled on those in [FU, Ch. 7], using the Continuity Method to solve the differential equation. The solution $a$ will be of the form $a=d_{+}^{*} b$ for $b \in \Lambda_{+}^{2}\left(\operatorname{End}_{0} E\right)$, where the subscript $A$ has been dropped to simplify the notation - throughout the proof, all differential operators acting on bundlevalued forms are those which are induced by the connection $A$ and the Riemannian connection induced by the metric, with $d_{0}$ denoting full covariant differentiation.

For $t \in[0,1]$ the equation $F_{+}\left(A+a_{t}\right)=(1-t) F_{+}(A)$ can be rewritten

$$
d_{+} d_{+}^{*} b_{t}+\left[a_{t}, d_{+}^{*} b_{t}\right]_{+}=-t F_{+}(A), \quad a_{t}=d_{+}^{*} b_{t}
$$

The Weitzenböck formula (6.26) of [FU] (with the orientation reversed) reads

$$
d_{+} d_{+}^{*}=d_{0}^{*} d_{0}+R / 3+\left[F_{+}(A), \cdot\right]
$$

where $R$ is the scalar curvature. If $R_{0}=\inf R$ and $F_{+}:=F_{+}(A)$ then for $b \in \Lambda_{+}^{2}$

$$
\begin{aligned}
\left\langle b, d_{+} d_{+}^{*} b+\left[a_{t}, b\right]_{+}\right\rangle & \geq\left\langle d_{0} b, d_{0} b\right\rangle+\frac{1}{3}\langle b, R b\rangle-\left(\left\|a_{t}\right\|_{L^{2}}+\left\|F_{+}\right\|_{L^{2}}\right)\|b\|_{L^{4}}^{2} \\
& \geq\left(c_{1}-2\left\|a_{t}\right\|_{L^{2}}-\left\|F_{+}\right\| L_{L^{2}}\right)\|b\|_{L^{4}}^{2}+\frac{R_{0}}{6}\|b\|_{L^{2}}^{2}
\end{aligned}
$$

where $c_{1}$ is the Sobolev constant such that $c_{1}\|f\|_{L^{4}}^{2} \leq\|d f\|_{L^{2}}^{2}+R_{0} / 6\|f\|_{L^{2}}^{2}$ for $f \in L_{1}^{2}(Y)$. Thus if $\left\|a_{t}\right\|_{L^{2}}+\left\|F_{+}\right\|_{L^{2}} \leq c_{1} / 2$, the linearisation of the operator $b \mapsto d_{+} d_{+}^{*} b+\left[d_{+}^{*} b, d_{+}^{*} b\right]_{+}$at $b=b_{t}$ has no kernel and is therefore an isomorphism.

Next, using (2.6) as above yields

$$
\begin{aligned}
\frac{1}{2} d^{*} d\left|b_{t}\right|^{2} & =-\left|d_{0} b_{t}\right|^{2}+\left\langle b_{t}, d_{0}^{*} d_{0} b_{t}\right\rangle \\
& \leq-\left|d_{0} b_{t}\right|^{2}-\frac{R_{0}}{3}\left|b_{t}\right|^{2}+t\left|b_{t}\right|\left|F_{+}\right|+t^{2}\left|b_{t}\right|^{2}\left|F_{+}\right|+\left|b_{t}\right|\left|a_{t}\right|^{2} \\
& \leq-\left(1-\left|b_{t}\right|\right)\left|d_{0} b_{t}\right|^{2}-\left(\frac{R_{0}}{3}-\delta\right)\left|b_{t}\right|^{2}+\left|b_{t}\right|\left|F_{+}\right|
\end{aligned}
$$

If $\sup \left|b_{t}\right| \leq 1$ and $\delta \leq R_{0} / 6$ then at the maximum of $\left|b_{t}\right|$ the left-hand side of this equation is non-negative giving the a priori bound

$$
\sup \left|b_{t}\right| \leq 6 \sup \left|F_{+}\right| / R_{0} .
$$


Moreover, if $\left|b_{t}\right| \leq 1 / 2$, the inequality above implies

$$
d^{*} d\left|b_{t}\right|^{2}+\left|d_{0} b_{t}\right|^{2}+\frac{R_{0}}{3}\left|b_{t}\right|^{2} \leq 2\left|b_{t}\right|\left|F_{+}\right|
$$

Integrating both sides also yields the a priori bound $\left\|a_{t}\right\|_{L^{2}}^{2} \leq\left\|d_{0} b_{t}\right\|_{L^{2}}^{2} \leq 2$ sup $\left|b_{t}\right|\left\|F_{+}\right\|_{L^{1}}$, so the invertiblity of the linearized operator in (2.5) is guaranteed by a suitable bound on sup $\left|F_{+}\right|$and the side condition $\sup \left|b_{t}\right| \leq 1 / 2$.

The Weitzenböck formula (6.25) of [FU] for 1-forms is

$$
\left.2 d_{+}^{*} d_{+}+d d^{*}=d_{0}^{*} d_{0}+R i c-2[\cdot\lrcorner F_{-}\right]
$$

where Ric is the Ricci curvature of the metric. Since $d^{*} a_{t}=0$ and $d_{+} a_{t}=-t F_{+}-a_{t} \wedge a_{t}$, it follows that

$$
\left.\frac{1}{2} d^{*} d\left|a_{t}\right|^{2}+\left|d_{0} a_{t}\right|^{2}=-\left\langle a_{t}, \operatorname{Ric}\left(a_{t}\right)\right\rangle+2\left\langle a_{t},\left[a_{t}\right\lrcorner F_{-}\right]\right\rangle-2\left\langle a_{t}, d_{+}^{*}\left(t F_{+}+\left(a_{t} \wedge a_{t}\right)_{+}\right)\right\rangle .
$$

Integrating both sides and estimating the right gives

$$
\begin{aligned}
\left\|d_{0} a_{t}\right\|_{L^{2}}^{2} & \leq\|R i c\|_{L^{2}}\left\|a_{t}\right\|_{L^{4}}^{2}+2\left\|a_{t}\right\|_{L^{4}}^{2}\|F\|_{L^{2}}+4\left\|a_{t}\right\|_{L^{4}}^{2}+4\left\|F_{+}\right\|_{L^{2}}^{2} \\
& \leq c_{2}\left\|a_{t}\right\|_{L^{4}}^{2}+4\left\|F_{+}\right\|_{L^{2}}^{2}
\end{aligned}
$$

where $c_{2}$ is a constant depending only on the Riemannian metric (and the Chern classes of $E$ assuming $\delta \leq 1$.). To deal with the $L^{4}$ norm of $a_{t}$ multiply $(2.8)$ through by $\left|a_{t}\right|^{2}$ and integrate. This gives

$$
\begin{aligned}
\int\left|a_{t}\right|^{2}\left|d_{0} b_{t}\right|^{2} d V & \leq 2 \int\left|b_{t}\right|\left|F_{+}\right|\left|a_{t}\right|^{2} d V-\int\left|b_{t}\right|^{2} d^{*} d\left|a_{t}\right|^{2} d V \\
& =2 \int\left|b_{t}\right|\left|F_{+}\right|\left|a_{t}\right|^{2} d V+2 \int\left|b_{t}\right|^{2}\left|d_{0} a_{t}\right|^{2} d V-2 \int\left|b_{t}\right|^{2} K d V
\end{aligned}
$$

where $K$ is the right-hand side of (2.9). That is,

$$
\left.-\int\left|b_{t}\right|^{2} K d V=\int\left|b_{t}\right|^{2}\left[\left\langle a_{t}, \operatorname{Ric}\left(a_{t}\right)\right\rangle-2\left\langle a_{t},\left[a_{t}\right\lrcorner F_{-}\right]\right\rangle\right] d V+\int\left|b_{t}\right|^{2}\left[\left\langle a_{t}, d_{+}^{*}\left(t F_{+}+\left(a_{t} \wedge a_{t}\right)_{+}\right)\right\rangle\right] d V .
$$

The first integral on the right-hand side is bounded by $c_{2} \sup \left|b_{t}\right|^{2}\left\|a_{t}\right\|_{L^{4}}^{2}$; the second is equal to

$$
\begin{aligned}
\int\left\langle d_{+}\left(\left|b_{t}\right|^{2} a_{t}\right), t F_{+}+\left(a_{t} \wedge a_{t}\right)_{+}\right\rangle d V & =-\left\|\left|b_{t}\right|\left[t F_{+}+\left(a_{t} \wedge a_{t}\right)_{+}\right]\right\|_{L^{2}}^{2} \\
& +\int\left\langle d\left|b_{t}\right|^{2} \wedge a_{t}, t F_{+}+\left(a_{t} \wedge a_{t}\right)_{+}\right\rangle d V \\
\leq & 2 \sup \left|b_{t}\right|\left\|\left|d_{0} b_{t}\left\|a_{t} \mid\right\|_{L^{2}}^{2}\left(1+\left\|F_{+}\right\|_{L^{2}}^{2}\right)\right.\right.
\end{aligned}
$$

using $\left|a_{t}\right| \leq\left|d_{0} b_{t}\right|$ and Young's Inequality. Substituting these bounds back into (2.11) and applying (2.10) then gives

$$
\begin{aligned}
\int\left|a_{t}\right|^{2}\left|d_{0} b_{t}\right|^{2} d V & \leq 2 \sup \left|b_{t}\right|\left[\left\|F_{+}\right\| L_{L^{2}}\left\|a_{t}\right\|_{L^{4}}^{2}+4 \sup \left|b_{t}\right|\left(c_{2}\left\|a_{t}\right\|_{L^{4}}^{2}+\left\|F_{+}\right\|_{L^{2}}^{2}\right)\right. \\
& +2\left\|\left|d_{0} b_{t}\left\|a_{t} \mid\right\|_{L^{2}}^{2}\left(1+\left\|F_{+}\right\|_{L^{2}}^{2}\right)\right]\right. \\
& \leq 4 \sup \left|b_{t}\right|\left[c_{3}\left\|\left|d_{0} b_{t}\left\|a_{t} \mid\right\|_{L^{2}}^{2}\right)+\right\| F_{+} \|_{L^{2}}^{2}\right]
\end{aligned}
$$

where $c_{3}$ depends only on the geometry. Rearranging terms gives

$$
\left(1-4 c_{3} \sup \left|b_{t}\right|\right) \int\left|a_{t}\right|^{2}\left|d_{0} b_{t}\right|^{2} d V \leq 4 \sup \left|b_{t}\right|^{2}\left\|F_{+}\right\|_{L^{2}}^{2}
$$

so if $\sup \left|b_{t}\right| \leq 1 / 8 c_{3}$ then $\left\|a_{t}\right\|_{L^{4}}^{4} \leq c_{4} \sup \left|F_{+}\right|^{4}$, using the bound on sup $\left|b_{t}\right|$ previously obtained. Feeding this estimate back into (2.10) then gives an a priori bound on $\left\|d_{0} a_{t}\right\|_{L^{2}}$ of the form $c_{5} \sup \left|F_{+}\right|$, and the remainder of the proof is a straight-forward application of the continuity method as in the proof of Theorem 7.27 of [FU]. The details will be omitted. 
It follows from Lemma 2.3 that there is a sequence of perturbations $\left\{a_{i}\right\}$ with $\left\|a_{i}\right\|_{L^{4}}+\left\|d_{\rho A_{i}, 0} a_{i}\right\|_{L^{2}}$ uniformly bounded by a constant of order $\sup \left|F_{+}\left(\rho A_{i}\right)\right|$ such that $\rho A_{i}+a_{i}$ is an anti-self-dual connection on an $S U(r)$ bundle over $S^{4}$, and the bound on $\left\|a_{i}\right\|_{L_{1}^{2}}$ implies that the transferred sequence is bubbling the same amount of charge at $0 \in \mathbb{C}^{2} \subset S^{4}$ as the original sequence was bubbling at $x_{0}, k>0$ units say.

\section{Curvature of instantons on $S^{4}$.}

The Atiyah-Ward correspondence between instantons on $S^{4}$ and monads on $\mathbb{C P}_{3}$ provides a description of the perturbed sequence of transferred connections in terms of unitary monads; that is, linear algebraic data. To construct "local" monads for the unperturbed sequence requires good estimates on the curvature of the instantons in terms of the corresponding monads, and obtaining such estimates is the object of this section.

Recall the description of anti-self-dual connections on $S^{4}$ in terms of holomorphic bundles and monads on $\mathbb{P}_{2}$ given in [D1]: every holomorphic $r$-bundle $E$ with $c_{2}=k$ which is trivial on the line $L_{\infty}$ at infinity is isomorphic to the cohomology of a monad of the form

$$
\mathbb{P}_{2}: \quad 0 \longrightarrow K(-1) \stackrel{A}{\longrightarrow} W \stackrel{B}{\longrightarrow} K(1) \longrightarrow 0 \quad,
$$

where $K$ and $W$ are hermitian vector spaces of dimension $k$ and $2 k+r$ respectively and $A, B$ are linear maps depending linearly on the homogeneous coordinates $Z \in \mathbb{P}_{2}: A(Z)=A_{0} Z^{0}+A_{1} Z^{1}+A_{2} Z^{2}$ and similarly for $B$. If the coordinates are chosen so that $L_{\infty}=\left\{Z=\left(Z^{0}, Z^{1}, Z^{2}\right) \mid Z^{2}=0\right\}$ then bases for $K, W$ can be chosen so that if $R$ is the $r$-dimensional vector space $R=\left(\operatorname{Im} A_{0}+\operatorname{Im} A_{1}\right)^{\perp}$ then $W=K \oplus K \oplus R$, $A\left(Z^{0}, Z^{1}, Z^{2}\right)=\left[\begin{array}{r}Z^{0} \mathbf{1}_{K}+Z^{2} a_{0} \\ Z^{1} \mathbf{1}_{K}+Z^{2} a_{1} \\ Z^{2} a_{2}\end{array}\right]$ and $B\left(Z^{0}, Z^{1}, Z^{2}\right)=\left[Z^{1} \mathbf{1}_{K}+Z^{2} a_{1},-Z^{0} \mathbf{1}_{K}-Z^{2} a_{0}, Z^{2} b_{2}\right]$ for some endomorphisms $a_{0}, a_{1}$ of $K$ and some $a_{2} \in \operatorname{Hom}(K, R), b_{2} \in \operatorname{Hom}(R, K)$. The monad condition becomes $a_{1} a_{0}-a_{0} a_{1}+b_{2} a_{2}=0$ and the non-singularity requirement is that $A(Z)$ be injective and $B(Z)$ be surjective at each point $Z \in \mathbb{P}_{2}$. The main result of [D1], proved using geometric invariant theory, is that each such monad is isomorphic to a corresponding monad on $\mathbb{P}_{3}$ restricted to the plane $Z^{3}=0$, where the latter monad possesses a unitary structure and corresponds to a self-dual Yang-Mills connection (instanton) on $S^{4}$. This is an alternate formulation of the result that every holomorphic bundle on $\mathbb{P}_{2}$ which is trivial on $L_{\infty}$ admits a hermitian connection which is anti-self-dual with respect to the flat metric on $\mathbb{C}^{2}$. Expressed in terms of the monads above, the unitary structure is equivalent to the condition that

$$
a_{0} a_{0}^{*}-a_{0}^{*} a_{0}+a_{1} a_{1}^{*}-a_{1}^{*} a_{1}+b_{2} b_{2}^{*}-a_{2}^{*} a_{2}=0 .
$$

Consider now the curvature of the induced unitary connection on the bundle $E$ which is the cohomology of the monad (3.1) (which is assumed to satisfy (3.2)). Let $\left(z^{0}, z^{1}\right)$ be inhomogeneous coordinates on $\mathbb{C}^{2}=\left\{Z \in \mathbb{P}_{2} \mid Z^{2} \neq 0\right\}$. Orthogonal projection $W \rightarrow E=\operatorname{ker} B \cap \operatorname{ker} A^{*}$ is then given by $\pi_{E}=$ $1-A\left(A^{*} A\right)^{-1} A^{*}-B^{*}\left(B B^{*}\right)^{-1} B$, from which it follows that the curvature of the induced connection on $E$ is $F=\left.\pi_{E}\left[d A\left(A^{*} A\right)^{-1} \wedge d A^{*}+d B^{*}\left(B B^{*}\right)^{-1} \wedge d B^{*}\right]\right|_{E}$. The unitary condition on the monad implies that $A^{*} A=B B^{*}=: \psi$, so if $i_{E}$ denotes the inclusion of $E$ into $W$ then the curvature is given explicitly by

$$
F=\pi_{E}\left[\begin{array}{ccc}
\psi^{-1} & 0 & 0 \\
0 & \psi^{-1} & 0 \\
0 & 0 & 0
\end{array}\right]\left[\begin{array}{ccc}
d z^{0} \wedge d \bar{z}^{0}-d z^{1} \wedge d \bar{z}^{1} & 2 d z^{0} \wedge d \bar{z}^{1} & 0 \\
2 d z^{1} \wedge d \bar{z}^{0} & d z^{1} \wedge d \bar{z}^{1}-d z^{0} \wedge d \bar{z}^{0} & 0 \\
0 & 0 & 0
\end{array}\right] i_{E}
$$

If $p_{i j}$ is the $i, j$-th block in $\pi_{E}$, then (3.3) gives

$$
* \operatorname{tr} F^{2}=8 \operatorname{tr}\left[\left(p_{00} \psi^{-1}+p_{11} \psi^{-1}\right)^{2}+2\left(p_{00} \psi^{-1} p_{11} \psi^{-1}-p_{01} \psi^{-1} p_{10} \psi^{-1}\right)\right] \leq 16 \operatorname{tr}\left[\left(p_{00} \psi^{-1}+p_{11} \psi^{-1}\right)^{2}\right] ;
$$

(the combinatorial factor 8 comes from the volume form $(1 / 4) d z^{0} \wedge d z^{1} \wedge d \bar{z}^{0} \wedge d \bar{z}^{1}$ ). Since $\pi_{E}$ and $\psi$ are positive semi-definite with the former a projection of $\operatorname{rank} r, * \operatorname{tr} F^{2} \leq 16\left[\operatorname{tr}\left(p_{00}+p_{11}\right)\right]^{2}\left[\operatorname{tr} \psi^{-1}\right]^{2}=$ $16\left[r-\operatorname{tr} p_{22}\right]^{2}\left[\operatorname{tr} \psi^{-1}\right]^{2} \leq 16 r^{2}\left[\operatorname{tr} \psi^{-1}\right]^{2}$. Thus

$$
|F| \leq 4 \operatorname{tr}\left(a_{2} \psi^{-1} a_{2}^{*}+b_{2}^{*} \psi^{-1} b_{2}\right) \operatorname{tr} \psi^{-1} \leq 4 r \operatorname{tr} \psi^{-1} .
$$


As the monad degenerates (that is, $\left.a_{0}, a_{1}, a_{2}, b_{2} \rightarrow 0\right)$ the automorphism $\psi \in E n d(K)$ approaches $|z|^{2} \mathbf{1}_{K}$. The following lemma provides the estimates required for the next section. In this lemma, the underlying Riemannian metric is the standard flat one.

Lemma 3.5. Let $f:=\left[\operatorname{tr} \psi^{-1}\right]^{-1 / 2}$ and $g:=\log \operatorname{det} \psi$. Then

(a) $|d f|^{2} \leq k$.

(b) $\triangle f^{-2}=4 \operatorname{tr}\left[\psi^{-1}\left(p_{00}+p_{11}\right) \psi^{-1}\right] \geq 0 \quad$ (where $p_{i j}$ is the $(i, j)$-th block of $\pi_{E}$ ).

(c) $|d g|^{2} \leq 4 k f^{-2}$.

(d) $\triangle g \leq-4 f^{-2}$.

(e) $\quad f\left|d^{2} f\right|+f^{2}\left|d^{2} g\right|$ is uniformly bounded by a combinatorial constant depending only on $k$.

Proof: (a) Set $\alpha_{i}:=z^{i}+a_{i}$ for $i=0,1$, so $A(z)=\left[\begin{array}{lll}\alpha_{0} & \alpha_{1} & a_{2}\end{array}\right]^{\mathrm{T}}$ and $d \psi=d\left(A^{*} A\right)=d A^{*} A+$ $A^{*} d A=\sum_{i}\left(\alpha_{i} d \bar{z}^{i}+\alpha_{i}^{*} d z^{i}\right)$. Since $d\left(\left(\operatorname{tr} \psi^{-1}\right)^{-1 / 2}\right)=(1 / 2)\left(\operatorname{tr} \psi^{-1}\right)^{-3 / 2} \operatorname{tr}\left[\psi^{-1}\left(d A^{*} A+A^{*} d A\right) \psi^{-1}\right]$ it follows $\left|d\left(\left(\operatorname{tr} \psi^{-1}\right)^{-1 / 2}\right)\right|^{2}=\left(\operatorname{tr} \psi^{-1}\right)^{-3} \sum_{i}\left|\operatorname{tr}\left(\psi^{-1} \alpha_{i} \psi^{-1}\right)\right|^{2} \leq\left(\operatorname{tr}\left(\psi^{-1}\right)\right)^{-3} \operatorname{tr}\left(\psi^{-3}\right) k \leq k$.

(b) $\quad-d \cdot d \operatorname{tr} \psi^{-1}=d \cdot\left(\operatorname{tr} \psi^{-1}\left(d A^{*} A+A^{*} d A\right) \psi^{-1}\right)=8 \operatorname{tr} \psi^{-2}-2 \operatorname{tr}\left[\psi^{-1}\left(d A^{*} A+A^{*} d A\right) \psi^{-1} \cdot\left(d A^{*} A+\right.\right.$ $\left.\left.A^{*} d A\right) \psi^{-1}\right]=8 \operatorname{tr} \psi^{-2}-4 \sum_{i} \operatorname{tr}\left[\psi^{-1}\left(\alpha_{i}^{*} \psi^{-1} \alpha_{i}+\alpha_{i} \psi^{-1} \alpha_{i}^{*}\right) \psi^{-1}\right]=4 \operatorname{tr}\left[\psi^{-1}\left(\mathbf{1}_{K}-\alpha_{0} \psi^{-1} \alpha_{0}^{*}-\alpha_{1}^{*} \psi^{-1} \alpha_{1}\right) \psi^{-1}+\right.$ $\left.\left.\psi^{-1}\left(\mathbf{1}_{K}-\alpha_{1} \psi^{-1} \alpha_{1}^{*}-\alpha_{0}^{*} \psi^{-1} \alpha_{0}\right) \psi^{-1}\right)\right]=4 \operatorname{tr}\left[\psi^{-1}\left(p_{00}+p_{11}\right) \psi^{-1}\right]$.

(c) $\quad d \log \operatorname{det} \psi=\operatorname{tr}\left[\psi^{-1}\left(d A^{*} A+A^{*} d A\right)\right]$, so $|d \log \operatorname{det} \psi|^{2}=\operatorname{tr}\left[\psi^{-1}\left(d A^{*} A+A^{*} d A\right)\right] \cdot \operatorname{tr}\left[\psi^{-1}\left(d A^{*} A+\right.\right.$ $\left.\left.A^{*} d A\right)\right]=4 \sum_{i}\left|\operatorname{tr} \psi^{-1} \alpha_{i}\right|^{2} \leq 4 k \operatorname{tr} \psi^{-1}$.

(d) $-d \cdot d \log \operatorname{det} \psi=-8 \operatorname{tr} \psi^{-1}+\operatorname{tr}\left[\psi^{-1}\left(d A^{*} A+A^{*} d A\right) \psi^{-1} \cdot\left(d A^{*} A+A^{*} d A\right)\right]=-8 \operatorname{tr} \psi^{-1}+$

$4 \sum_{i} \operatorname{tr}\left[\psi^{-1} \alpha_{i} \psi^{-1} \alpha_{i}^{*}\right]=-4 \operatorname{tr} \psi^{-1}-2 \operatorname{tr}\left[\psi^{-1}\left(p_{00}+p_{11}\right)\right] \leq-4 \operatorname{tr} \psi^{-1}$.

(e) Straightforward calculation as above.

Lemma 3.6. If $0 \leq p<2, q \geq 0$, and $c$ is a constant with $c \geq g+\frac{2 k(2 q-2+p)}{2-p}$ in a ball $B$ then

$$
\triangle\left[f^{-p}(c-g)^{q}\right] \geq 2 q f^{-p-2}(c-g)^{q-1} \quad \text { in } B
$$

Proof: By direct calculation and using Lemma 3.5,

$$
\begin{aligned}
\triangle\left[f^{-p}(c-g)^{q}\right] & =\triangle\left(f^{-p}\right)(c-g)^{q}+f^{-p} \triangle(c-g)^{q}-2 d\left(f^{-p}\right) \cdot d(c-g)^{q}, \\
\triangle\left(f^{-p}\right) & =(p / 2) f^{2-p} \triangle\left(f^{-2}\right)+p(2-p) f^{-p-2}|d f|^{2} \\
& \geq p(2-p) f^{-p-2}|d f|^{2} \\
\triangle(c-g)^{q} & =-q(c-g)^{q-1} \triangle g-q(q-1)(c-g)^{q-2}|d g|^{2} \\
& \geq 4 q(c-g)^{q-1} f^{-2}-q(q-1)(c-g)^{q-2}|d g|^{2} .
\end{aligned}
$$

By Young's Inequality,

$$
\begin{aligned}
2 d\left(f^{-p}\right) \cdot d(c-g)^{q} & =2 p q f^{-p-1}(c-g)^{q-1} d f \cdot d g \\
& \leq p(2-p)(c-g)^{q} f^{-p-2}|d f|^{2}+\frac{p q^{2}}{2-p}(c-g)^{q-2} f^{-p}|d g|^{2}
\end{aligned}
$$

Combining the above and using Lemma 3.5(c) to estimate $|d g|^{2}$ gives $\triangle\left[f^{-p}(c-g)^{q}\right] \geq 4 q f^{-p-2}(c-g)^{q-2}(c-$ $\left.g-\frac{k(2 q-2+p)}{2-p}\right)$ from which the desired inequality follows.

Remark: Integrating the inequality of the lemma over the unit ball shows that the function $f^{-2}(c-g)^{q}=$ $\operatorname{tr} \psi^{-1}(c-\log \operatorname{det} \psi)^{q}$ is uniformly bounded in $L^{p}$ for any $p<2$ and $q \geq 0$ as $\left|a_{0}\right|+\left|a_{1}\right|+\left|a_{2}\right| \rightarrow 0$. This fails for general $a_{0}, a_{1}, a_{2}$ not satisfying the reality constraint (3.2) required to correspond to an instanton.

Suppose now that the monad (3.1) corresponds to an anti-self-dual connection on a bundle over $S^{4}$ which has been obtained from the procedure of $\S 2$ : a concentrated Hermitian-Einstein connection $A$ on $(X, \omega)$ has been cut off and transferred to $\rho A$ on $S^{4}$, then perturbed to an anti-self-dual connection $\rho A+a$ on an $S U(r)$ bundle over $S^{4}$ with $c_{2}=k$, with $a=d_{\rho A}^{+*} b$. The cutoff function $\rho$ is identically 1 on a neighbourhood of $\bar{B}(0,3)$ and vanishes outside $B(0,4)$. 
The following lemma provides useful estimates on the size of the perturbation $a$ in terms of the monad (3.1) and the automorphism $\psi$ :

Lemma 3.7. There is a constant $C_{0}$ independent of $A$ such that if $\eta=\sup \left|F_{+}(\rho A)\right|$ is sufficiently small, then

$$
|a|^{2} \leq C_{0}^{2} \eta^{2} \operatorname{tr} \psi^{-1} \quad \text { in } B(0,3)
$$

Proof: The underlying Riemannian metric is the standard metric on $S^{4}$, and $d_{0}$ is the full covariant derivative induced by $\rho A$ and this metric. The calculations of $\S 2$ yield bounds similar to (2.8) of the form

$$
\triangle|b|^{2}+\left|d_{0} b\right|^{2} \leq 2|b|\left|F_{+}(\rho A)\right|, \quad|b| \leq c_{1} \eta
$$

for some constant $c_{1}$ independent of $A$.

The Weitzenböck Formula (6.25) of [FU] gives $\left.\triangle_{0} a=-\operatorname{Ric}(a)-2 d_{+}^{*}\left[F_{+}(\rho A)-(a \wedge a)_{+}\right]+2[a\lrcorner F_{-}(\rho A)\right]$, so

$$
\begin{aligned}
\triangle|a|^{2}+2\left|d_{0} a\right|^{2}=2\left\langle a, \triangle_{0} a\right\rangle & \leq 4|a|\left|d_{+}^{*} F_{+}(\rho A)\right|+4|F(\rho A)||a|^{2}+8|a|^{2}\left|d_{0} a\right| \\
& \leq 4|a|\left|d_{+}^{*} F_{+}(\rho A)\right|+4|F(\rho A+a)||a|^{2}+12|a|^{2}\left|d_{0} a\right|+4|a|^{4}
\end{aligned}
$$

Fix a smooth cutoff function $\chi$ which is identically 1 on $B(0,3)$ and which is supported $\{z \in B(0,4) \mid$ $\rho(z)=1\}$. With $f:=\left[\operatorname{tr} \psi^{-1}\right]^{-1 / 2}$ as in Lemma 3.5 it follows from that lemma that both $\left|\triangle\left(\chi^{2} f^{2}\right)\right|,|d(\chi f)|$ are uniformly bounded by a fixed constant, so $\triangle\left[\chi^{2} f^{2}|a|^{2}\right]=\triangle\left(\chi^{2} f^{2}\right)|a|^{2}+\chi^{2} f^{2} \triangle|a|^{2}-2\left\langle d\left(\chi^{2} f^{2}\right), d|a|^{2}\right\rangle \leq$ Const. $\left(|a|^{2}+\chi f|a|\left|d_{0} a\right|\right)+\chi^{2} f^{2} \triangle|a|^{2}$. Since $F_{+}(\rho A) \equiv 0$ in $B(0,3)$ and $|F(\rho A+a)| \leq C o n s t . f^{-2}$ there, (3.10) implies that $\triangle\left[\chi^{2} f^{2}|a|^{2}\right]+2 \chi^{2} f^{2}\left|d_{0} a\right|^{2} \leq$ Const. $\left(|a|^{2}+\chi f|a|\left|d_{0} a\right|\right)+12|a|^{2} \chi^{2} f^{2}\left|d_{0} a\right|+4 \chi^{2} f^{2}|a|^{4}$. Applying Young's inequality, it follows that there is a constant $c_{2}$ independent of $A$ such that

$$
\triangle\left[\chi^{2} f^{2}|a|^{2}\right]+\chi^{2} f^{2}\left|d_{0} a\right|^{2} \leq c_{2}\left(|a|^{2}+\chi^{2} f^{2}|a|^{4}\right)
$$

If $Q:=\sup (\chi f|a|)$ then $\triangle\left[\chi^{2} f^{2}|a|^{2}\right] \leq c_{2}(1+Q)|a|^{2}$, so by (3.9) it follows that $\triangle\left[\chi^{2} f^{2}|a|^{2}+c_{2}(1+\right.$ $\left.Q)|b|^{2}\right] \leq 0$. By the Maximum Principle and (3.9), $\chi^{2} f^{2}|a|^{2}+c_{2}(1+Q)|b|^{2} \leq c_{2}(1+Q) c_{1}^{2} \eta^{2}$ implying in particular that $Q^{2} \leq c_{1}^{2} c_{2} \eta^{2}(1+Q)$ and yielding the desired result.

Remark: By differentiating again and expressing $d_{0} F(\rho A)$ in terms of $d_{\rho A+a, 0} F(\rho A+a)$ (which is of order $f^{-3}$ ) and $d_{0} a$, it is straightforward to show that the same methods yield a bound on $\left|d_{0} a\right|$ of the form $\left|d_{0} a\right| \leq$ Const. $\eta \operatorname{tr} \psi^{-1}$ in $B(0,2)$, and therefore there is a bound of the form $|F(\rho A)| \leq C$ onst. $\eta$. tr $\psi^{-1}$ in $B(0,2)$.

\section{Perturbation of monads I.}

Let $(A, \rho, a)$ be as at the end of $\S 3$, and let $E$ be the unitary $r$-bundle over $S^{4}$ on which $\rho A+a$ is defined. The construction of a monad on $\mathbb{P}_{2}$ (restricted from $\mathbb{P}_{3}$ ) corresponding to the instanton $\rho A+a$ can be viewed as a particular embedding of the bundle $E$ into a larger topologically trivial bundle which is equipped with a flat connection so that the connection on $E$ is that which is induced by Hermitian projection. That is, splitting the bundle $W$ of (3.1) using the Hermitian metric gives an isomorphism of $W$ with $K(-1) \oplus E \oplus K(1)$ so that the connection $d_{W}$ on $W$ is identified as

$$
\left[\begin{array}{c}
K(-1) \\
E \\
K(1)
\end{array}\right] \ni\left[\begin{array}{c}
p \\
q \\
r
\end{array}\right] \mapsto\left[\begin{array}{c}
d_{K(-1)} p \\
d_{E} q \\
d_{K(1)} r
\end{array}\right]+\left[\begin{array}{ccc}
0 & \alpha & \beta \\
-\alpha^{*} & 0 & \gamma \\
-\beta^{*} & -\gamma^{*} & 0
\end{array}\right]\left[\begin{array}{c}
p \\
q \\
r
\end{array}\right]
$$

where $\alpha \in \Lambda^{(0,1)} \otimes \operatorname{Hom}(E, K(-1)), \beta \in \Lambda^{(0,1)} \otimes \operatorname{Hom}(K(1), K(-1))$ and $\gamma \in \Lambda^{(0,1)} \otimes H o m(K(1), E)$ are the $\bar{\partial}$-closed $(0,1)$-forms representing the extensions; i.e., the second fundamental forms.

In this and the next section it will be shown that the perturbation $a$ of the connection $\rho A$ is induced by a corresponding small perturbation of the flat connection on $W$. Since no curvature can bubble from a sequence of nearly-flat connections on $W$, the degeneration of the connections on $E$ in a neighbourhood of 0 will be manifested as the degeneration of the holomorphic maps $K(-1) \rightarrow W, W \rightarrow K(1)$, where $W$ is now 
equipped with its new holomorphic structure. This degeneration is then amenable to analysis by algebraic methods.

Because the procedure for constructing the perturbation of the monad is quite lengthy and somewhat indirect, the method is outlined below.

Let $d_{W}^{\prime}$ be the connection on $W$ which is constructed from the connection $\rho A$ on $E$ together with the connections on $K( \pm 1)$ induced by (4.1) using the same second fundamental forms; that is (abusing notation slightly), $d_{W}^{\prime}=d_{W}-a$. Thus

$$
F\left(d_{W}^{\prime}\right)=F\left(d_{W}\right)-d_{W} a+a \wedge a=\left[\begin{array}{ccc}
0 & -\alpha \wedge a & 0 \\
a \wedge \alpha^{*} & -d_{\rho A} a-a \wedge a & -a \wedge \gamma \\
0 & \gamma^{*} \wedge a & 0
\end{array}\right] .
$$

The first perturbation of $d_{W}^{\prime}$ which is sought will be obtained from a perturbation $(\alpha+\delta \alpha, \beta+\delta \beta, \gamma+\delta \gamma)$ of $(\alpha, \beta, \gamma)$ so that the only non-zero $(0,2)+(2,0)$ component of the curvature is that coming from $F(\rho A)$. Using (4.2), this means that with the connections on $K( \pm 1)$ fixed and with the connection $\rho A$ on $E$, the equations to be satisfied are

$$
\begin{aligned}
\bar{\partial} \delta \alpha & =\alpha \wedge a^{\prime \prime} \\
\bar{\partial} \delta \beta & =-[\alpha \wedge \delta \gamma+\delta \alpha \wedge \gamma+\delta \alpha \wedge \delta \gamma] \\
\bar{\partial} \delta \gamma & =-a^{\prime \prime} \wedge \gamma
\end{aligned}
$$

where $a^{\prime \prime}$ is the $(0,1)$ component of $a$.

The system (4.3) is non-linear only in an elementary way and can be solved via linear equations, first solving (a) and (c), and then (b). The standard approach would be to take $(\delta \alpha, \delta \beta, \delta \gamma)=\left(\bar{\partial}^{*} x, \bar{\partial}^{*} y, \bar{\partial}^{*} z\right)$, where adjoints are with respect to the Fubini-Study metric on $\mathbb{P}_{2}$. For any of the $\bar{\partial}$-operators involved in the system, the kernel of $\triangle^{\prime \prime}$ on $\Lambda^{0,2}$ is canonically dual to the kernel of $\triangle^{\prime \prime}$ on sections of the dual bundle tensored with the canonical bundle $\mathcal{O}(-3)$ of $\mathbb{P}_{2}$. Since $\rho A$ is flat outside $B(0,4)$, any such section of the latter on $\mathbb{P}_{2} \backslash B(0,4)$ is a section of $\mathcal{O}(-3)$ tensored with a trivial bundle twisted by $\mathcal{O}(1)$ or $\mathcal{O}(2)$. Such a section vanishes on all lines not meeting $B(0,4)$ and hence everywhere, being the solution of an elliptic equation. Therefore, since $\triangle^{\prime \prime}$ has index 0 , there is no obstruction to solving (4.3)-indeed, $(x, y, z)$ are uniquely determined.

Having thus perturbed the connection $d_{W}^{\prime}$ to $d_{W}^{\prime \prime}$ say, the second step is to remove the central part of the curvature: an inspection of the proof of the main result of [B4] shows that the methods there remain valid in the current setting, even though the connection is not everywhere integrable. That is, there is a solution $g \in A u t_{\mathbb{C}}(W)$ on $\mathbb{C}^{2}$ to the equation $\widehat{F}\left(g \cdot d_{W}^{\prime \prime}\right)=0$ (where the $\Lambda$ operator $\Lambda^{1,1} \rightarrow \Lambda^{0,0}$ is now that coming from the flat metric on $\mathbb{C}^{2}$ ), with $g^{*} g \rightarrow \mathbf{1}$ along $L_{\infty}$. The self-dual component of the curvature of this new connection is now $g F^{0,2}(\rho A) g^{-1}+g^{*-1} F^{2,0}(\rho A) g^{*}$ (where notation has again been abused by suppressing the inclusion map of $E$ into $W$ and projection map $W \rightarrow E$ ). To ensure that this remains uniformly bounded and small therefore requires that the automorphism $g$ and its inverse remain uniformly bounded in the annulus supporting $F_{+}(\rho A)$. Such a bound can be obtained from an appropriate bound on $\widehat{F}\left(d_{W}^{\prime \prime}\right)$, which in turn follows from good estimates on the solutions of (4.3). The problem outstanding is to provide these estimates.

On a compact Kähler surface, the Weitzenböck formulae relating the $\bar{\partial}$-Laplacian $\triangle^{\prime \prime}=\bar{\partial} \bar{\partial}^{*}+\bar{\partial}^{*} \bar{\partial}$ associated with a connection on a bundle $E$ with the full covariant Laplacian $\triangle_{0}$ are

$$
\triangle_{0}=\left\{\begin{array}{lll}
2 \triangle^{\prime \prime}+i \widehat{F} & \text { on } & \Lambda^{0,0}(E) \\
2 \triangle^{\prime \prime}+2 i \Lambda F_{-} \wedge-i \Lambda R \wedge & \text { on } & \Lambda^{0,1}(E) \\
2 \triangle^{\prime \prime}-i \widehat{F}-i \widehat{R} & \text { on } & \Lambda^{0,2}(E)
\end{array}\right.
$$

where $R \in \Lambda^{1,1}$ is the Ricci curvature and $F$ is the curvature of the connection. (The equations themselves are easily deduced from the Hodge Identities, using the fact that on $\Lambda^{0, q}$, the exterior derivative $\partial$ agrees with the full covariant derivative $\partial_{0}$.)

The connections on $K(-1)$ and $\operatorname{Hom}(K(1),-)$ induced from the monad have curvatures $F$ such that $i F$ is negative, becoming highly so in the ball $B(0,4)$ as the monad degenerates. This sign makes estimation of solutions to (4.3) less than straightforward, and forces a modification to the strategy outlined above. The approach taken here is to solve $(4.3)$ in $B(0,3)$ using Dirichlet boundary conditions and the flat Riemannian metric, for which the solutions are more easily estimated. These solutions can then be cut off with small 
error to yield a smooth almost-flat connection on $W$ over $S^{4}$ to which the remainder of the method can be applied.

With $A$ and $B$ as in (3.1) the second fundamental forms in (4.1) can be identified as

$$
\alpha=-\left.\left(A^{*} A\right)^{-1 / 2} \bar{\partial} A^{*}\right|_{E}, \quad \beta=\left(A^{*} A\right)^{-1 / 2} A^{*}\left(\bar{\partial} B^{*}\right)\left(B B^{*}\right)^{-1 / 2}, \quad \gamma=\pi_{E}\left(\bar{\partial} B^{*}\right)\left(B B^{*}\right)^{-1 / 2}
$$

where the adjoints are with respect to the Hermitian metric on $W$ and a fixed metric on $K$, the line bundle $\mathcal{O}(-1)$ assumed to have been trivialised over the affine portion of $\mathbb{P}_{2}$. Thus $|\alpha|^{2},|\beta|^{2},|\gamma|^{2} \leq 4 \operatorname{tr} \psi^{-1}$, with $\psi=A(z)^{*} A(z)$ as before.

Consider first the equation (4.3)(a): $\bar{\partial} \delta \alpha=\alpha \wedge a^{\prime \prime}$, where the $\bar{\partial}$-operator is that which is induced on $\operatorname{Hom}(E, K(-1))$ by the connection $\rho A$ on $E$ and that which is induced by Hermitian projection from the monad (3.1) on $K(-1)$. If $\bar{\partial}_{u}$ denotes the standard $\bar{\partial}$-operator on the trivial bundle $K$ over $\mathbb{C}^{2}$ (the subscript indicates "untwisted"), then $\bar{\partial}=\psi^{1 / 2} \circ \bar{\partial}_{u} \circ \psi^{-1 / 2}$ so (4.3)(a) is equivalent to the equation

$$
\bar{\partial}_{u}\left(\psi^{-1 / 2} \delta \alpha\right)=\psi^{-1 / 2} \alpha \wedge a^{\prime \prime} .
$$

(The twisting by $\mathcal{O}(-1)$ has been taken into account here: $\bar{\partial}=\Psi^{1 / 2} \circ \bar{\partial}^{\prime} \circ \Psi^{-1 / 2}$ where $\Psi:=A(Z)^{*} A(Z) /|Z|^{2}$ and $\bar{\partial}^{\prime}$ is the standard $\bar{\partial}$-operator on $\mathcal{O}(-1)$ over $\mathbb{P}_{2}$.) As indicated above, the solution of this last equation is obtained by solving the Dirichlet problem

$$
\left(\triangle_{u}^{\prime \prime} \tau=\right) \quad \bar{\partial}_{u} \bar{\partial}_{u}^{*} \tau=\psi^{-1 / 2} \alpha \wedge a^{\prime \prime} \quad \text { in } B(0,3), \tau=0 \text { on } \partial B(0,3)
$$

for $\tau \in \Lambda^{0,2}(\operatorname{Hom}(E, K(-1))$. Since all sections here are smooth, standard linear elliptic PDE theory gives a smooth solution of the equation, but the key information required are estimates on such a solution. In the following lemma, $d_{u 0}$ denotes the full covariant derivative induced from the flat metric on $\mathbb{C}^{2}$ together with $d_{u}$ on $K(-1)$ and $\rho A$ on $E$.

Lemma 4.7. If $\left|a_{0}\right|+\left|a_{1}\right|+\left|a_{2}\right| \leq 1$ and $\eta$ is sufficiently small, the solution $\tau$ of (4.6) satisfies

$$
\begin{aligned}
|\tau|^{2} & \leq C_{1}^{2} \eta^{2} \operatorname{tr} \psi^{-1} \\
\triangle\left|\psi^{1 / 2} \tau\right|^{2}+\left|\psi^{1 / 2} d_{u 0} \tau\right|^{2} & \leq C_{1}^{2} \eta^{2} \operatorname{tr} \psi^{-1} \\
\left|\psi^{1 / 2} \tau\right|^{2} & \leq C_{1}^{2} \eta^{2}(3 k-\log \operatorname{det} \psi)
\end{aligned}
$$

where $C_{1}$ is a constant independent of $A$.

Proof: As in $\S 3$, let $f=\left[\operatorname{tr} \psi^{-1}\right]^{-1 / 2}$ and $g=\log \operatorname{det} \psi$. The third Weitzenböck formula of (4.4) with $\widehat{F}=0=\widehat{R}$ gives

$$
\triangle|\tau|^{2}+2\left|d_{u 0} \tau\right|^{2} \leq 2|\tau|\left|\triangle_{u 0} \tau\right|=4|\tau|\left|\psi^{-1 / 2} \alpha \wedge a^{\prime \prime}\right| .
$$

By Lemma 3.5, the bound on $|\alpha|$ and the Cauchy-Schwarz inequality, it follows that $\triangle|\tau| \leq\left|\triangle_{0} \tau\right| \leq$ $2\left|\psi^{-1 / 2} \alpha \wedge a^{\prime \prime}\right| \leq 2 c_{1} \eta f^{-3}$ for some constant $c_{1}$ independent of the connection $A$.

The assumption $\left|a_{0}\right|+\left|a_{1}\right|+\left|a_{2}\right| \leq 1$ implies that $g \leq 3 k$ in $B(0,3)$. Taking $c=5 k$ and $p=1=q$ in Lemma 3.6 gives $\triangle\left[f^{-1}(5 k-g)\right] \geq 2 f^{-3}$ in $B(0,3)$, so by the Maximum Principle it follows that

$$
|\tau| \leq c_{1} \eta f^{-1}(5 k-g) .
$$

To sharpen this estimate, observe that

$$
\left|\psi^{-1 / 2} \alpha\right|^{2}=\operatorname{tr}\left[\pi_{E} d A \psi^{-2} \cdot d A^{*}\right]=2 \operatorname{tr}\left[\psi^{-2}\left(p_{00}+p_{11}\right)\right]=(1 / 2) \triangle \operatorname{tr} \psi^{-1},
$$

(where $p_{i j}$ is the $i, j$-th block in $\pi_{E}$ with respect to the decomposition $W=K \oplus K \oplus \mathbb{C}^{r}$ ). If $\lambda>0$ is a constant (to be fixed later) it follows from (4.11) that

$$
\begin{aligned}
\triangle|\tau|^{2}+2\left|d_{u 0} \tau\right|^{2} & \leq 4|\tau|\left|a^{\prime \prime}\right|\left|\psi^{-1 / 2} \alpha\right| \\
& \leq 2 \lambda^{-1} \eta^{2}\left|\psi^{-1 / 2} \alpha\right|^{2}+\left.2 \lambda \eta^{-2}|\tau|^{2}|| a^{\prime \prime}\right|^{2} \\
& =\lambda^{-1} \eta^{2} \triangle \operatorname{tr} \psi^{-1}+\lambda \eta^{-2}|\tau|^{2}|a|^{2} .
\end{aligned}
$$

As in (3.9), there is an inequality of the form

$$
\triangle|b|^{2}+\left|d_{0} b\right|^{2} \leq 2|b|\left|F_{+}(\rho A)\right|+c_{2}|b|^{2}=c_{2}|b|^{2} \quad \text { in } B(0,3)
$$


for some uniform constant $c_{2}$ independent of $A$, this additional term arising from the fact that it is now the flat metric on $\mathbb{C}^{2}$ which is being used rather than the conformally flat metric on $S^{4}$; note that implies $|a|^{2}=\left|*_{S^{4}} d b\right|^{2} \leq\left(1+|z|^{2}\right)^{4}\left|d_{0} b\right|^{2}$. Using Young's inequality,

$$
\begin{aligned}
\triangle\left(|b|^{2}|\tau|^{2}\right) & =\triangle\left(|b|^{2}\right)|\tau|^{2}+|b|^{2} \triangle|\tau|^{2}-2 d|b|^{2} \cdot d|\tau|^{2} \\
& \leq-\left|d_{0} b\right|^{2}|\tau|^{2}-2|b|^{2}\left|d_{u 0} \tau\right|^{2}+|b|^{2}\left[c_{2}|\tau|^{2}+\lambda^{-1} \eta^{2} \triangle \operatorname{tr} \psi^{-1}+\lambda \eta^{-2}|\tau|^{2}|a|^{2}\right]+8|b||\tau|\left|d_{0} b\right|\left|d_{u 0} \tau\right| \\
& \leq-(1 / 2)\left|d_{0} b\right|^{2}|\tau|^{2}+30|b|^{2}\left|d_{u 0} \tau\right|^{2}+|b|^{2}\left[c_{2}|\tau|^{2}+\lambda^{-1} \eta^{2} \triangle \operatorname{tr} \psi^{-1}+\lambda \eta^{-2}|\tau|^{2}|a|^{2}\right] .
\end{aligned}
$$

If $c_{3}$ is a fixed uniform constant such that $|b| \leq c_{3} \eta$ in $B(0,3)$ then from (4.14),

$$
\triangle\left[|b|^{2}|\tau|^{2}+16 c_{3}^{2} \eta^{2}|\tau|^{2}\right] \leq-\left(1 / 2-32 \lambda c_{3}^{2}\right)\left|d_{0} b\right|^{2}|\tau|^{2}-2 c_{3}^{2} \eta^{2}\left|d_{u 0} \tau\right|^{2}+c_{2} c_{3}^{2} \eta^{2}|\tau|^{2}+17 \lambda^{-1} c_{3}^{2} \eta^{4} \triangle \operatorname{tr} \psi^{-1},
$$

so if $\lambda=1 /\left(128 c_{3}^{2}\right)$ it follows that

$$
\triangle\left[|b|^{2}|\tau|^{2}+16 c_{3}^{2} \eta^{2}|\tau|^{2}\right]+(1 / 4)\left|d_{0} b\right|^{2}|\tau|^{2}+2 c_{3}^{2} \eta^{2}\left|d_{u 0} \tau\right|^{2} \leq c_{2} c_{3}^{2} \eta^{2}|\tau|^{2}+17 \lambda^{-1} c_{3}^{2} \eta^{4} \triangle \operatorname{tr} \psi^{-1} .
$$

By (4.12) and Lemma 3.6, $|\tau|^{2} \leq c_{1}^{2} \eta^{2} f^{-2}(5 k-g)^{2} \leq \triangle\left[c_{1}^{2} \eta^{2}(7 k-g)^{3} / 6\right]$, so by the Maximum Principle it follows that $|b|^{2}|\tau|^{2}+16 c_{3}^{2} \eta^{2}|\tau|^{2} \leq 17 \lambda^{-1} c_{3}^{2} \eta^{4} \operatorname{tr} \psi^{-1}+c_{1}^{2} c_{3}^{2} \eta^{4}(7 k-g)^{3} / 6$. The hypotheses imply that $(7 k-g)^{3}$ is bounded in $B(0,3)$ by a uniform constant times $\operatorname{tr} \psi^{-1}$, implying in particular that $|\tau|^{2} \leq$ Const. $\eta^{2} \operatorname{tr} \psi^{-1}$ for some constant independent of $A$, proving (4.8).

To prove (4.9) and (4.10), $\triangle\left|\psi^{1 / 2} \tau\right|^{2}=\triangle\langle\tau, \psi \tau\rangle=2 \operatorname{Re}\left\langle\tau, \psi \triangle_{u 0} \tau\right\rangle-4 \operatorname{Re}\left\langle d_{u 0} \tau,(d \psi) \tau\right\rangle-8|\tau|^{2}-$ $2\left|\psi^{1 / 2} d_{u 0} \tau\right|^{2}$. By the Weitzenböck formula, $\triangle_{u 0} \tau=2 \triangle_{u}^{\prime \prime} \tau=2 \psi^{-1 / 2} \alpha \wedge a^{\prime \prime}$, so

$$
\triangle\left|\psi^{1 / 2} \tau\right|^{2}+\left|\psi^{1 / 2} d_{u 0} \tau\right|^{2} \leq 4 \operatorname{Re}\left\langle\tau, \psi^{1 / 2} \alpha \wedge a^{\prime \prime}\right\rangle+8|\tau|^{2} \leq \text { Const. } \eta^{2} \operatorname{tr} \psi^{-1},
$$

where the second inequality follows from the last bound on $|\tau|$ and Lemma 3.8. The bound (4.10) on $\left|\psi^{1 / 2} \tau\right|$ then follows from the Maximum Principle and Lemma 3.5(c).

Equation $(4.3)$ (c) can be solved in $B(0,3)$ in precisely the same way as above; the solution $\delta \gamma$ takes the form $\delta \gamma=\left(\bar{\partial}_{u}^{*} \mu\right) \psi^{1 / 2}$ for some $\mu \in \Lambda^{0,2}(\operatorname{Hom}(K(1), E)$ satisfying the same estimates as $\tau$ in Lemma 4.7; (more precisely, $\mu^{*}$ does). Equation (4.3)(b) is however more delicate: using the untwisted connection on $\operatorname{Hom}(K(1), K(-1))$ it takes the form

$$
\bar{\partial}_{u}\left(\psi^{-1 / 2} \delta \beta \psi^{-1 / 2}\right)=-\psi^{-1 / 2}[\alpha \wedge \delta \gamma+\delta \alpha \wedge \gamma+\delta \alpha \wedge \delta \gamma] \psi^{-1 / 2}
$$

where $\delta \alpha=\psi^{1 / 2} \bar{\partial}_{u}^{*} \mu$ and $\delta \gamma=\left(\bar{\partial}_{u}^{*} \nu\right) \psi^{1 / 2}$. As above the equation can be solved in $B(0,3)$ by taking $\delta \beta=\psi^{1 / 2} \bar{\partial}_{u}^{*} \nu \psi^{1 / 2}$ for $\nu \in \Lambda^{(0,2)}(\operatorname{Hom}(K(1), K(-1)))$ vanishing on $\partial B(0,3)$, but complications arise from the fact that the right-hand side of the equation

$$
\triangle_{u}^{\prime \prime} \nu=-\left[\psi^{-1 / 2} \alpha \wedge \bar{\partial}_{u}^{*} \mu+\bar{\partial}_{u}^{*} \tau \wedge \gamma \psi^{-1 / 2}+\bar{\partial}_{u}^{*} \tau \wedge \bar{\partial}_{u}^{*} \mu\right]
$$

is of order $f^{-4}$ making application of Lemma 3.6 impossible. However, using (4.13) as was done in the proof of Lemma 4.7 overcomes this problem.

Lemma 4.17. If $\left|a_{0}\right|+\left|a_{1}\right|+\left|a_{2}\right| \leq 1$ and $\eta$ is sufficiently small, the solution $\nu$ of (4.16) satisfies

$$
\begin{aligned}
|\nu|^{2} & \leq C_{2}^{2} \eta^{2} f^{-2} \\
\triangle\left[\left|\psi^{1 / 2} \nu \psi^{1 / 2}\right|^{2}+c_{0}(5 k-g)\left(\left|\psi^{1 / 2} \tau\right|^{2}+\left|\mu \psi^{1 / 2}\right|^{2}\right)\right]+\left|\psi^{1 / 2} d_{u 0} \nu \psi^{1 / 2}\right|^{2} & \leq C_{2}^{2} \eta^{2}(5 k-g)^{2} f^{-2} \\
\left|\psi^{1 / 2} \nu \psi^{1 / 2}\right|^{2} & \leq C_{2}^{2} \eta^{2}(5 k-g)^{2}
\end{aligned}
$$

where $c_{0}, C_{2}$ are constants independent of the connection $A$, and $f^{-2}=\operatorname{tr} \psi^{-1}$ and $g=\log \operatorname{det} \psi$.

Proof: As in the proof of Lemma 4.7,

$$
\triangle|\nu| \leq\left|\triangle_{u 0} \nu\right|=2\left|\triangle_{u}^{\prime \prime} \nu\right| \leq \eta\left(\left|\psi^{-1 / 2} \alpha\right|^{2}+\left|\gamma \psi^{-1 / 2}\right|^{2}\right)+2 \eta^{-1}\left(\left|\bar{\partial}_{u}^{*} \tau\right|^{2}+\left|\bar{\partial}_{u}^{*} \mu\right|^{2}\right) .
$$

From (4.15) and the corresponding inequality for $\mu$ it follows that

$$
\triangle\left[|\nu|+\eta^{-3} c_{3}^{-2}\left(|b|^{2}+16 c_{3}^{2} \eta^{2}\right)\left(|\tau|^{2}+|\mu|^{2}\right)\right] \leq c_{4}\left[\eta^{-1}\left(|\tau|^{2}+|\mu|^{2}\right)+\eta \triangle \operatorname{tr} \psi^{-1}\right]
$$


for some uniform constant $c_{4}$, so by (4.8), (4.13) and the Maximum Principle it follows as above that $|\nu| \leq c_{5} \eta \operatorname{tr} \psi^{-1}$.

Inequality (4.19) will follow once (4.20) has been established:

$$
\begin{aligned}
\triangle\left|\psi^{1 / 2} \nu \psi^{1 / 2}\right|^{2}= & \triangle\langle\nu, \psi \nu \psi\rangle \\
= & \left\langle\triangle_{u 0} \nu, \psi \nu \psi\right\rangle+\left\langle\nu, \psi\left(\triangle_{u 0} \nu\right) \psi\right\rangle-8\langle\nu, \psi \nu+\nu \psi\rangle-2\left\langle d_{u 0} \nu, \psi\left(d_{u 0} \nu\right) \psi\right\rangle \\
& -2\left\langle d_{u 0} \nu,(d \psi) \nu \psi+\psi \nu d \psi\right\rangle-2\left\langle(d \psi) \nu,\left(d_{u 0} \nu\right) \psi\right\rangle-2\left\langle\nu d \psi, \psi d_{u 0} \nu\right\rangle-2\langle(d \psi) \nu, \nu d \psi\rangle .
\end{aligned}
$$

Setting $X:=\psi^{1 / 2} d_{u 0} \nu \psi^{1 / 2}, Y:=\psi^{-1 / 2}(d \psi) \psi^{-1 / 2}$ and $Z:=\psi^{1 / 2} \nu \psi^{1 / 2}$, the terms involving a factor of -2 in this equation are $\left.-2\left[|X|^{2}+\langle X, Y Z+Z Y\rangle+\langle Y Z+Z Y, X\rangle+\langle Y Z, Z Y\rangle\right] \leq 2|Z Y|^{2}+2|Z Y|^{2}+2\langle Z Y, Y Z\rangle\right]$. Since $|Z Y|^{2}=\operatorname{tr} \psi^{-1 / 2} d \psi \nu^{*} \psi \nu \cdot d \psi \psi^{-1 / 2}=2 \operatorname{tr}\left[\nu^{*} \psi \nu\left(\mathbf{1}_{K}-p_{00}+\mathbf{1}_{K}-p_{11}\right)\right] \leq 4\langle\nu, \psi \nu\rangle$ and similarly $|Y Z|^{2} \leq 4\langle\nu, \nu \psi\rangle$, it follows that

$$
\begin{aligned}
\triangle\left|\psi^{1 / 2} \nu \psi^{1 / 2}\right|^{2} & \leq\left\langle\triangle_{u 0} \nu, \psi \nu \psi\right\rangle+\left\langle\nu, \psi\left(\triangle_{u 0} \nu\right) \psi\right\rangle+2\langle\nu d \psi,(d \psi) \nu\rangle \\
& \leq 4\left|\psi^{1 / 2} \nu \psi^{1 / 2}\right|\left|\psi^{1 / 2}\left(\triangle_{u}^{\prime \prime} \nu\right) \psi^{1 / 2}\right|+2\left|\psi^{1 / 2} \nu \psi^{1 / 2}\right|\left|\psi^{-1 / 2} d \psi\right|\left|\psi^{-1 / 2} d \psi \| \nu\right| \\
& \leq 4\left|\psi^{1 / 2} \nu \psi^{1 / 2}\right|\left[|\alpha \wedge \delta \gamma+\delta \alpha \wedge \gamma+\delta \alpha \wedge \delta \gamma|+c_{6} \eta f^{-2}\right] \\
& \leq 4 Q(5 k-g)\left[\eta^{-1}|\delta \alpha|^{2}+\eta^{-1}|\delta \gamma|^{2}+c_{7} \eta f^{-2}\right] \quad \text { for } Q:=\sup \frac{\left|\psi^{1 / 2} \nu \psi^{1 / 2}\right|}{5 k-g}
\end{aligned}
$$

where $c_{6}, c_{7}$ are constants independent of $A$. From (4.9), (4.10) and Lemma 3.5,

$$
\triangle\left[(5 k-g)\left|\psi^{1 / 2} \tau\right|^{2}\right]+\frac{1}{2}(5 k-g)\left|\psi^{1 / 2} d_{u 0} \tau\right|^{2} \leq c_{8} \eta^{2} f^{-2}(5 k-g),
$$

so

$$
\triangle\left[\left|\psi^{1 / 2} \nu \psi^{1 / 2}\right|^{2}+8 Q \eta^{-1}(5 k-g)\left(\left|\psi^{1 / 2} \tau\right|^{2}+\left|\mu \psi^{1 / 2}\right|^{2}\right)\right] \leq 16 Q c_{8} \eta f^{-2}(5 k-g) .
$$

By Lemma 3.6 again,

$$
\triangle\left[\left|\psi^{1 / 2} \nu \psi^{1 / 2}\right|^{2}+8 Q \eta^{-1}(5 k-g)\left(\left|\psi^{1 / 2} \tau\right|^{2}+\left|\mu \psi^{1 / 2}\right|^{2}\right)-4 Q c_{8} \eta(5 k-g)^{2}\right] \leq 0,
$$

implying in particular that $\left|\psi^{1 / 2} \nu \psi^{1 / 2}\right|^{2} \leq 4 Q c_{8} \eta(5 k-g)^{2}$. Hence $\left|\psi^{1 / 2} \nu \psi^{1 / 2}\right|^{2}(5 k-g)^{-2} \leq 4 Q c_{8} \eta$, giving $Q \leq 4 c_{8} \eta$; that is,

$$
\left|\psi^{1 / 2} \nu \psi^{1 / 2}\right| \leq 4 c_{8} \eta(5 k-g) .
$$

Note that this implies $\left|\nu \psi^{1 / 2}\right|,\left|\psi^{1 / 2} \nu\right| \leq 4 c_{8} \eta(5 k-g) f^{-1}$.

Returning now to the proof of (4.19),

$$
\begin{aligned}
\triangle\left|\psi^{1 / 2} \nu \psi^{1 / 2}\right|^{2}+\left|\psi^{1 / 2} d_{u 0} \nu \psi^{1 / 2}\right|^{2} & \leq 4\left|\psi^{1 / 2} \nu \psi^{1 / 2}\right||\alpha \wedge \delta \gamma+\delta \alpha \wedge \gamma+\delta \alpha \wedge \delta \gamma|+c_{9}\left(\left|\nu \psi^{1 / 2}\right|^{2}+\left|\psi^{1 / 2} \nu\right|^{2}\right) \\
& \leq c_{10}\left[\eta(5 k-g)|\alpha \wedge \delta \gamma+\delta \alpha \wedge \gamma+\delta \alpha \wedge \delta \gamma|+\eta^{2}(5 k-g)^{2} f^{-2}\right] \\
& \leq c_{11}\left[(5 k-g)\left(|\delta \alpha|^{2}+|\delta \gamma|^{2}\right)+\eta^{2}(5 k-g)^{2} f^{-2}\right]
\end{aligned}
$$

and therefore, as above it follows that

$$
\triangle\left[\left|\psi^{1 / 2} \nu \psi^{1 / 2}\right|^{2}+2 c_{11}(5 k-g)\left(\left|\psi^{1 / 2} \tau\right|^{2}+\left|\mu \psi^{1 / 2}\right|^{2}\right)\right]+\left|\psi^{1 / 2} d_{u 0} \nu \psi^{1 / 2}\right|^{2} \leq c_{12} \eta^{2}(5 k-g)^{2} f^{-2},
$$

as required.

Remark: Using the fact that the curvature of $\rho A$ is bounded by a constant times $\operatorname{tr} \psi^{-1}$ as remarked at the end of $\S 3$, the same methods as given here yield bounds on $|\delta \alpha|,|\delta \gamma|,|\delta \beta|$ over $\bar{B}(0,2)$ of the form Const. $\eta f^{-1}(c-g)^{q}$ for some (fixed) $q$; indeed, the majority of the work has already been done. Furthermore, it is easily shown that $\left|d_{0} \delta \alpha\right|,\left|d_{0} \delta \gamma\right|,\left|d_{0} \delta \beta\right|$ are uniformly bounded in $L^{2}(B(0,2))$ by a term of order $\eta$.

\section{Perturbation of monads II.}


Consider now the curvature of the perturbed connection $d_{W}^{\prime \prime}$ on $W$ over the ball $B(0,3)$ constructed in the last section:

$$
\left[\begin{array}{cc}
\left(-\alpha \wedge \delta \alpha^{*}-\beta \wedge \delta \beta^{*}-\delta \alpha \wedge \alpha^{*}\right. & \left(d \delta \alpha-\alpha \wedge a-\beta \wedge \delta \gamma^{*}\right. \\
\left.-\delta \beta \wedge \beta^{*}-\delta \alpha \wedge \delta \alpha^{*}-\delta \beta \wedge \delta \beta^{*}\right) & \left.-\delta \beta \wedge \gamma^{*}-\delta \beta \wedge \delta \gamma^{*}\right) \\
\left(-d \delta \alpha^{*}+a \wedge \alpha^{*}-\delta \gamma \wedge \beta^{*}\right. & \left(-\left[d_{\rho A} a+a \wedge a\right]-\alpha^{*} \wedge \delta \alpha-\gamma \wedge \delta \gamma^{*}\right. \\
\left.-\gamma \wedge \delta \beta^{*}-\delta \gamma \wedge \delta \beta^{*}\right) & \left.-\delta \alpha^{*} \wedge \alpha-\delta \gamma \wedge \gamma^{*}-\delta \alpha^{*} \wedge \delta \alpha-\delta \gamma \wedge \delta \gamma^{*}\right) \\
\left(-d \delta \beta^{*}+\delta \gamma^{*} \wedge \alpha^{*}\right. & \left(d \delta \gamma^{*}+\gamma^{*} \wedge a-\delta \beta^{*} \wedge \alpha\right. \\
\left.+\gamma^{*} \wedge \delta \alpha+\delta \gamma^{*} \wedge \delta \alpha^{*}\right) & \left.-\beta^{*} \wedge \delta \alpha-\delta \beta^{*} \wedge \delta \alpha\right)
\end{array}\right.
$$$$
\left.\begin{array}{c}
(d \delta \beta+\alpha \wedge \delta \gamma \\
+\delta \alpha \wedge \gamma+\delta \alpha \wedge \delta \gamma) \\
\left(d \delta \gamma-a \wedge \gamma-\alpha^{*} \wedge \delta \beta\right. \\
\left.-\delta \alpha^{*} \wedge \beta-\delta \alpha^{*} \wedge \delta \beta\right) \\
\left(-\gamma^{*} \wedge \delta \gamma-\delta \gamma^{*} \wedge \gamma-\beta^{*} \wedge \delta \beta\right. \\
\left.-\delta \beta^{*} \wedge \beta-\delta \beta^{*} \wedge \delta \beta-\delta \gamma^{*} \wedge \delta \gamma\right)
\end{array}\right]
$$

By construction, the $(0,2)$ and $(2,0)$ components of $F\left(d_{W}^{\prime \prime}\right)$ are zero. Since both $\rho A$ and $\rho A+a$ are anti-selfdual in $B(0,3)$ it follows that $\left|\widehat{F}\left(d_{W}^{\prime \prime}\right)\right| \leq C\left[\eta^{-1}\left(|\delta \alpha|^{2}+|\delta \beta|^{2}+|\delta \gamma|^{2}\right)+\eta f^{-2}\right]+2(|\Lambda \partial \delta \alpha|+|\Lambda \partial \delta \beta|+|\Lambda \partial \delta \gamma|)$, where the $\partial$-operators here are the respective twisted connections induced from the monad (3.1) and $\rho A$, and $C$ is a combinatorial constant.

To estimate the terms involving derivatives of the perturbations, observe that

$$
i \Lambda \partial \delta \alpha=i \Lambda \psi^{-1 / 2} \partial_{u}\left(\psi^{1 / 2} \psi^{1 / 2} \bar{\partial}_{u}^{*} \tau\right)=-\psi^{-1 / 2} \bar{\partial}_{u}^{*}\left(\psi \bar{\partial}_{u}^{*} \tau\right)=i \Lambda\left(\psi^{-1 / 2} \partial \psi \psi^{-1 / 2} \wedge \delta \alpha\right),
$$

so it follows that

$$
|\Lambda \partial \delta \alpha| \leq\left|\psi^{-1 / 2} \partial \psi \psi^{-1 / 2}\right||\delta \alpha| \leq \eta\left|\psi^{-1 / 2} \partial \psi \psi^{-1 / 2}\right|^{2}+\eta^{-1}|\delta \alpha|^{2} \leq 2 \eta \operatorname{tr} \psi^{-1}+\eta^{-1}|\delta \alpha|^{2} .
$$

Similarly, $i \Lambda \partial \delta \gamma=-i \Lambda \delta \gamma \wedge \psi^{-1 / 2} \partial \psi \psi^{-1 / 2}$ and $i \Lambda \partial \delta \beta=i \Lambda\left[\psi^{-1 / 2} \partial \psi \psi^{-1 / 2} \wedge \delta \beta-\delta \beta \wedge \psi^{-1 / 2} \partial \psi \psi^{-1 / 2}\right]$, so the same type of estimate holds for $|\Lambda \partial \delta \beta|$ and $|\Lambda \delta \gamma|$. Thus for some new constant $C$,

$$
\left|\widehat{F}\left(d_{W}^{\prime \prime}\right)\right| \leq C\left[\eta^{-1}\left(|\delta \alpha|^{2}+|\delta \beta|^{2}+|\delta \gamma|^{2}\right)+\eta f^{-2}\right] .
$$

Now fix a smooth cutoff function $\chi$ compactly supported in $B(0,3)$ which is identically 1 on a neighbourhood of $\bar{B}(0,2)$, and replace $\tau, \mu$ and $\nu$ by $\chi \tau, \chi \mu$ and $\chi \nu$ respectively. Equations (4.9) and (4.19) remain true if the constants $C_{1}$ and $C_{2}$ are altered, and if $\eta$ is sufficiently small (or the connection $\rho A$ is sufficiently concentrated) the resulting perturbation to $d_{W}^{\prime \prime}$ is small in $C^{1}$, bounded by a term of order $\eta \sup _{B(0,3) \backslash B(0,2)}|\alpha|$.

Consider the connection on $W$ over $\mathbb{C}^{2}$ obtained by cutting off $\tau, \mu$ and $\nu$ in this way and replacing $\rho A$ by $\rho A+(1-\rho) a$. Outside $B(0,4)$, it agrees with the original flat connection $d_{W}$ on $W$; the $(0,2)$ and $(2,0)$ components of the curvature are supported in the annulus $B(0,4) \backslash \bar{B}(0,2)$ and are bounded in $C^{0}$ by a term of order $\eta$; and on a neighbourhood of $\bar{B}(0,2)$ it agrees with $d_{W}^{\prime \prime}$ and therefore induces the connection $\rho A$ on $E$ by hermitian projection. The notation $d_{W}^{\prime \prime}$ will be retained for this new connection.

As noted in the introduction to the previous section, the methods of [B4] can be applied to $d_{W}^{\prime \prime}$ to give a smooth solution $g \in A u t_{\mathbb{C}}(W)$ to the equation $\widehat{F}\left(g \cdot d_{W}^{\prime \prime}\right)=0$ with with $\operatorname{det} g \equiv 1$ and $g^{*} g \rightarrow \mathbf{1}_{W}$ on the line $L_{\infty}$ at infinity in $\mathbb{P}_{2}$. Then $\triangle \log |g|^{2}=2 i \Lambda \bar{\partial} \partial \log |g|^{2} \leq 2\left|\widehat{F}\left(d_{W}^{\prime \prime}\right)\right|$, so from (5.1), (4.9) and (4.19) (with modified constants to account for the introduction of $\chi$ ) it follows that in a neighbourhood of $\bar{B}(0,3)$,

$$
\triangle\left[\log |g|^{2}+2 C \eta^{-1}\left(1+c_{0}(5 k-\log \operatorname{det} \psi)\right)\left(\left|\psi^{1 / 2} \tau\right|^{2}+\left|\mu \psi^{1 / 2}\right|^{2}\right)+\left|\psi^{1 / 2} \mu \psi^{1 / 2}\right|^{2}\right] \leq C_{3} \eta f^{-2}(5 k-\log \operatorname{det} \psi)^{2}
$$

where $C_{3}$ is a constant independent of $\rho A$. Applying Lemma 3.6 with $p=0, q=3$ gives $\triangle\left((7 k-\log \operatorname{det} \psi)^{3}\right) \geq$ $6 f^{-2}(7 k-\log \operatorname{det} \psi)^{2}$ in $B(0,3)$. If $(7 k-g)_{+}$denotes the positive part of the function $7 k-g$ then for some suitable constant $C_{4}$ it follows

$$
\triangle\left[(7 k-\log \operatorname{det} \psi)_{+}^{3}+C_{4}\left(1+|z|^{2}\right)^{-1}\right] \geq 6 f^{-2}(7 k-\log \operatorname{det} \psi)_{+}^{2} \quad \text { in } \mathbb{C}^{2} .
$$

Therefore, by the Maximum Principle and the fact that $g^{*} g \rightarrow 1$ as $|z| \rightarrow \infty$ it follows that

$$
\log |g|^{2} \leq \log r+C_{5} \eta\left[(7 k-\log \operatorname{det} \psi)_{+}^{3}+\left(1+|z|^{2}\right)^{-1}\right]
$$

for some uniform constant $C_{5}$. Since $\operatorname{det} g=1$, a corresponding uniform bound exists for $g^{-1}$, and since $F_{+}\left(g \cdot d_{W}^{\prime \prime}\right)=g F^{0,2}\left(d_{W}^{\prime \prime}\right) g^{-1}+g^{*-1} F^{2,0}\left(d_{W}^{\prime \prime}\right) g^{*}$ it follows that $\left|F_{+}\left(g \cdot d_{W}^{\prime \prime}\right)\right| \leq$ Const. $\eta$. Topological triviality of $W$ implies $\left\|F\left(g \cdot d_{W}^{\prime \prime}\right)\right\|_{L^{2}} \leq$ Const. $\eta$ and therefore if $\eta$ is sufficiently small it follows from Uhlenbeck's 
theorem [U1], [S] that after gauge transformations, (a subsequence of) the connections $g \cdot d_{W}^{\prime \prime}$ converges in $C^{2}$ (say) on $\mathbb{C}^{2}$ to a smooth connection on $W$ which is anti-self-dual in the complement of the annulus $B(0,4) \backslash \bar{B}(0,2)$.

Since the sequence of connections $g \cdot d_{W}^{\prime \prime}$ is converging in $C^{2}$ and each defines an integrable connection in a neighbourhood of $\bar{B}(0,2)$, there is a converging sequence of holomorphic trivialisations of each of these connections in this neighbourhood. Equivalently, there is a convergent sequence of smooth automorphisms $g^{\prime}$ of $W$ there such that $g^{\prime} \cdot\left(g \cdot d_{W}^{\prime \prime}\right)=d_{W}$. Using a cutoff function supported in $B(0,3)$ which is the identity on $B(0,2)$, extend $g^{\prime}$ to $\mathbb{C}^{2}$ and combine the product of automorphisms $g^{\prime} g$ into one, also denoted by $g$. Thus $g \cdot d_{W}^{\prime \prime}=d_{W}$ in $B(0,2), g^{*} g \rightarrow 1$ along $L_{\infty}$, $\operatorname{det} g=1$ (without loss of generality) and the sequence of such automorphisms is converging uniformly in $C^{3}$ (say) on compact subsets of $\mathbb{C}^{2} \backslash\{0\}$.

As the sequence of connections degenerates, the cohomology of the monad (3.1) converges to the trivial rank $r$ bundle $R$ on compact subsets of $\mathbb{C}^{2} \backslash\{0\}$, and moreover the second fundamental forms $\alpha, \beta, \gamma$ converge to zero on such subsets - equations (4.6) and (4.16) together with a priori $L^{2}$ estimates from (4.12) and (4.18) imply that the perturbations $\delta \alpha, \delta \beta, \delta \gamma$ also converge to 0 uniformly on compact subsets of $B(0,3) \backslash\{0\}$.

The perturbation $a$ of the cut-off connection $\rho A$ satisfies $\left[d_{\rho A+a} a-a \wedge a\right]_{+}=0$ inside $B(0,3)$ and in view of the a priori $L_{1}^{2}$ bounds on $a$, it can be assumed to converge weakly in $L_{1}^{2}\left(\mathbb{C}^{2}\right)$ and uniformly in $C^{1}$ on compact subsets of $\mathbb{C}^{2} \backslash\{0\}$ to a form $a_{\infty}$ satisfying $\left[d_{R} a_{\infty}-a_{\infty} \wedge a_{\infty}\right]_{+}=0$. Regarding $a_{\infty}$ as defining a connection on $R$, the Removable Singularities Theorem [U2] gives a gauge in which $a_{\infty}$ extends smoothly across the origin. Combining the Newlander-Nirenberg theorem with this gauge transformation yields an automorphism $g_{\infty}^{\prime}$ of $R$ with unit determinant which is smooth away from the origin such that $a_{\infty}^{(0,1)}=\left(\bar{\partial}_{R} g_{\infty}^{\prime}\right) g_{\infty}^{\prime-1}$. Away from 0 , extend $g_{\infty}^{\prime}$ to all of $W$ by setting $\left.g_{\infty}^{\prime}\right|_{R^{\perp}}=\mathbf{1}_{R^{\perp}}$, so $g_{\infty}^{\prime} \cdot d_{W, \infty}^{\prime \prime}=d_{W}$ in $B(0,2)$. If $g_{\infty}$ is the limit of the sequence of automorphisms constructed in the previous paragraph, then $g_{\infty} \cdot d_{W, \infty}^{\prime \prime}=d_{W}$ in $B(0,2) \backslash\{0\}$ also, so $g_{\infty} g_{\infty}^{\prime-1}$ is a holomorphic automorphism of $W$ with unit determinant over $B(0,2) \backslash\{0\}$ which extends smoothly across the origin by Hartogs' Theorem. Using a cutoff function which is the identity on $B(0,3 / 2)$ and is compactly supported in $B(0,2)$, extend $h_{\infty}$ smoothly to all $\mathbb{C}^{2}$ so that it has unit determinant everywhere and is the identity outside $B(0,2)$, and then replace each of the automorphisms $g$ of the sequence by $h_{\infty}^{-1} g$. Then inside $B(0,3 / 2)$, the identity $g \cdot d_{W}^{\prime \prime}=d_{W}$ is still valid, each automorphism $g$ still has unit determinant, $g$ is converging in $C^{2}$ on compact subsets of $\mathbb{C}^{2} \backslash\{0\}, g^{*} g \rightarrow 1$ on $L_{\infty}$, and inside $B(0,3 / 2) \backslash\{0\}$ the limit restricts to the identity on $E^{\perp}$.

Recall that by construction of the connection $d_{W}^{\prime \prime}$, the maps $K(-1) \rightarrow W, W \rightarrow K(1)$ of the monad (3.1) are holomorphic with respect to this connection in $B(0,2)$. Thus, in this ball the connection $\rho A$ on $E$ is induced by Hermitian projection from the monad

$$
M^{\prime \prime}: \quad 0 \longrightarrow K(-1) \stackrel{A}{\longrightarrow} \stackrel{d_{W}^{\prime \prime}}{W} \stackrel{B}{\longrightarrow} K(1) \longrightarrow 0
$$

where the notation indicates that $W$ is equipped with the connection $d_{W}^{\prime \prime}$. Since $g \cdot d_{W}^{\prime \prime}=d_{W}$ in $B(0,3 / 2)$, it follows that $g A(z): K(-1) \rightarrow W$ and $B(z) g^{-1}: W \rightarrow K(1)$ are holomorphic (with respect to the standard $\bar{\partial}$ operators). Indeed the connection $\rho A$ on $E$ is precisely the "pull-back" of the induced hermitian connection on the cohomology of the monad

$$
M^{\prime}: \quad 0 \longrightarrow K(-1) \quad \stackrel{g A}{\longrightarrow} \stackrel{d_{W}}{W} \stackrel{B g^{-1}}{\longrightarrow} K(1) \longrightarrow 0
$$

meaning that if $E\left(M^{\prime \prime}\right), E\left(M^{\prime}\right)$ are the cohomologies of the respective monads, then

$$
E\left(M^{\prime \prime}\right)=\operatorname{ker} B / \operatorname{im} A \ni e+A K(-1) \mapsto g e+g A K(-1) \in \operatorname{ker} B g^{-1} / \operatorname{im} g A=E\left(M^{\prime}\right)
$$

defines a smooth map such that $\left(\bar{\partial}_{\rho A}=\right) \bar{\partial}^{\prime \prime}=g \circ \bar{\partial}^{\prime} \circ g^{-1}$ and $\left(\partial_{\rho A}=\right) \partial^{\prime \prime}=g^{*-1} \circ \partial^{\prime} \circ g^{*}$, where the hermitian structures on each of the bundles is that which is induced by hermitian projection from the flat metric on $W$. Choosing a unitary isomorphism between $E\left(M^{\prime \prime}\right)$ and $E\left(M^{\prime}\right)$ gives a hermitian connection on $E\left(M^{\prime \prime}\right)$ of the form $g_{1} \cdot \rho A$ for some complex automorphism $g_{1}$. Since the sequences $\{A(z)\},\{B(z)\}$ converge to limits which are non-degenerate away from the origin and the sequences $\{g\},\left\{g^{-1}\right\}$ are uniformly bounded on compact subsets of the complement of the origin, the same holds for $\left\{g_{1}\right\}$ and $\left\{g_{1}^{-1}\right\}$.

The fact that the automorphisms $g$ in the sequence are converging to the identity on $E^{\perp}$ away from the origin implies that the holomorphic maps $g A$ and $B g^{-1}$ are converging to the standard degenerate forms $A_{1}:=\left[\begin{array}{lll}z^{0} & z^{1} & 0\end{array}\right]^{T}, B_{1}:=\left[\begin{array}{lll}z^{1} & -z^{0} & 0\end{array}\right]$ respectively. A priori this convergence is on compact subsets of $B(0,3 / 2) \backslash\{0\}$, but since these maps are holomorphic it follows from Cauchy's Theorem (Removable Singularities) that the convergence is in fact throughout all of $B(0,3 / 2)$. 
The next step is to further modify the automorphisms $g$ so that the holomorphic maps $\widetilde{A}:=g A$ and $\widetilde{B}:=B g^{-1}$ have a more convenient form.

Proposition 5.5. Let $D:=\left\{\left(z^{0}, z^{1}\right) \in \mathbb{C}^{2}|| z^{0}|<1,| z^{1} \mid<1\right\}$ be the unit polydisk and let $A_{1}:=$ $\left[\begin{array}{lll}z_{0} \mathbf{1} & z^{1} \mathbf{1} & 0\end{array}\right]^{T} \in \operatorname{Hom}(K, W), B_{1} ;=\left[\begin{array}{lll}z_{1} 1 & -z^{0} \mathbf{1} & 0\end{array}\right] \in \operatorname{Hom}(W, K)$. If $\epsilon:=\sup _{D}\left[\left|\widetilde{A}-A_{1}\right|+\left|\widetilde{B}-B_{1}\right|\right]$ is sufficiently small there exists a holomorphic matrix $h$ on $D$ together with a constant matrix $\beta \in$ End $K$ with $\sup _{D}|h-1|+|\beta-1| \leq C_{0} \epsilon$ such that $h \widetilde{A}=A_{1}+A_{0}$ and $\widetilde{B} h^{-1}=\beta\left(B_{1}+B_{0}\right)$ for some constant $A_{0}$ and $B_{0}$.

Proof: The proof boils down to an application of the Implicit Function Theorem, for which a little preparation is required.

Let $\mathcal{H}$ be the set of functions holomorphic in a neighbourhood of $\bar{D}$ and let $\overline{\mathcal{H}}$ be its completion under the norm $\|*\|$ given by $\|f\|:=\sup _{D}|f|$, so $\overline{\mathcal{H}}$ is a Banach algebra. If $f \in \mathcal{H}$, there is a unique function $f_{1} \in \mathcal{H}$ such that $f\left(z^{0}, z^{1}\right)-f\left(z^{0}, 0\right)=z^{1} f_{1}\left(z^{0}, z^{1}\right)$. The maximum of $\left|f_{1}\right|$ on $D$ is attained at a point $(a, b)$ where $|a|=1$ or $|b|=1$, but if $|a|=1$, then since $f_{1}\left(a, z^{1}\right)$ is holomorphic in $z^{1}$ this function attains its maximum on the unit disk at a point where $\left|z^{1}\right|=1$, so $\left|f_{1}\right|$ always attains its maximum at a point where $\left|z^{1}\right|=1$. Hence $\left\|f_{1}\right\|=\left\|z^{1} f_{1}\right\| \leq 2\|f\|$. If $\left\{f_{i}\right\} \in \mathcal{H}$ is a Cauchy sequence converging to $f \in \overline{\mathcal{H}}$, then $\left\{\left(f_{i}\right)_{1}\right\}$ is Cauchy and converges to $f_{1}$. It follows that the map $\overline{\mathcal{H}} \ni f \mapsto f_{1} \in \overline{\mathcal{H}}$ is a $C^{1}$ map. Similarly, for a function $f=f\left(z^{0}\right)$ alone, the decomposition $f=f(0)+z^{0} f_{0}\left(z^{0}\right)$ gives a differentiable function $\overline{\mathcal{H}} \ni f \mapsto f_{0} \in \overline{\mathcal{H}}$.

Now let $\mathcal{A}, \mathcal{B}$ respectively be the spaces of linear maps $K \rightarrow W, W \rightarrow K$ with coefficients in $\mathcal{H}$, and let $\mathcal{G}$ be the space of endomorphisms of $W$ with coefficients in $\mathcal{H}$ which, with respect to the decomposition $W=[K \oplus K \oplus R]^{T}$ have the block form

$$
h=\left[\begin{array}{lll}
h_{00} & h_{01} & h_{02} \\
h_{10} & h_{11} & h_{12} \\
h_{20} & h_{21} & h_{22}
\end{array}\right]=\left[\begin{array}{lll}
h_{00} & h_{01} & h_{02} \\
h_{10,0}\left(z^{0}\right)+z^{1} h_{10,1}\left(z^{0}\right) & h_{11} & h_{12}\left(z^{0}\right) \\
h_{20}\left(z^{0}\right) & h_{21} & 0
\end{array}\right] .
$$

Let $\mathcal{C}$ be the space of triples $\left(A, \beta_{0}, \beta_{2}\right) \in \operatorname{Hom}(K, W) \oplus \operatorname{Hom}(K, K) \oplus \operatorname{Hom}(R, K)$ with coefficients in $\mathcal{H}$ such that $A(0,0)=0, \beta_{2}(0,0)=0$, and if $A=\left[\begin{array}{lll}z^{0} \alpha_{0,0}\left(z^{0}\right)+z^{1} \alpha_{0,1} & z^{0} \alpha_{1,0}\left(z^{0}\right)+z^{1} \alpha_{1,1} & \alpha_{2}\end{array}\right]^{T}$ then $\beta_{0}\left(z^{0}, z^{1}\right)=z^{0} \alpha_{1,0}+z^{1} \beta_{0,1}$ for some $\beta_{0,1}$ satisfying $\beta_{0,1}(0,0)=-\alpha_{0,0}(0)$. Define $P: \mathcal{A} \times \mathcal{B} \rightarrow C$ by

$$
P(A, B):=\left(A-A(0)-A_{1}, z^{0} \alpha_{1,0}+z^{1}\left(\mathbf{1}_{K}-\alpha_{0,0}(0)+\beta_{0,1}-\beta_{0,1}(0,0)\right), \beta_{2}-\beta_{2}(0)\right)
$$

for $A=\left[\begin{array}{lll}z^{0} \alpha_{0,0}\left(z^{0}\right)+z^{1} \alpha_{0,1} & z^{0} \alpha_{1,0}\left(z^{0}\right)+z^{1} \alpha_{1,1} & \alpha_{2}\end{array}\right]^{T}$ and $B=\left[\begin{array}{lll}\beta_{0} & \beta_{1} & \beta_{2}\end{array}\right]$.

Now define a map $F$ from a neighbourhood of $\left(A_{1}, B_{1}, 0\right) \in \mathcal{A} \times \mathcal{B} \times \mathcal{G}$ to $\mathcal{C}$ by

$$
F(A, B, h):=P\left((1+h) A, B(1+h)^{-1}\right) .
$$

Then $F\left(A_{1}, B_{1}, 0\right)=(0,0,0)$ and the partial derivative of $F$ in the $\mathcal{G}$-direction at $\left(A_{1}, B_{1}, 0\right)$ is given by

$$
\mathcal{G} \ni h \mapsto\left(\left[\begin{array}{c}
z^{0} h_{00}+z^{1} h_{01} \\
z^{0} h_{10}+z^{1} h_{11} \\
z^{0} h_{20}+z^{1} h_{21}
\end{array}\right],-\left(z^{1} h_{00}-z^{0} h_{10}\right),-\left(z^{1} h_{02}-z^{0} h_{12}\right)\right) \in \mathcal{C}
$$

after a short calculation to check on the second component.

If $\left(A, \beta_{0}, \beta_{1}\right) \in \mathcal{C}$ has the form

$$
\left(\left[\begin{array}{c}
z^{0} \alpha_{0,0}+z^{1} \alpha_{0,1} \\
z^{0} \alpha_{1,0}+z^{1} \alpha_{1,1} \\
z^{0} \alpha_{2,0}+z^{1} \alpha_{2,1}
\end{array}\right], z^{0} \alpha_{1,0}+z^{1} \beta_{0,1}, z^{0} \beta_{2,0}+z^{1} \beta_{2,1}\right)
$$

for some $\beta_{0,1}$ such that $\beta_{0,1}(0,0)=-\alpha_{0,0}(0)$ then there is a unique solution $h$ of the form (5.6) mapped by (5.7) onto $\left(A, \beta_{0}, \beta_{1}\right)$, namely

$$
h=\left[\begin{array}{llc}
\alpha_{0,0}-z^{1} \beta_{0,11} & \alpha_{0,1}+z^{0} \beta_{0,11} & -\beta_{2,1} \\
\alpha_{1,0}+z^{1}\left(\alpha_{0,00}+\beta_{0,10}\right) & \alpha_{1,1}-z^{0}\left(\alpha_{0,00}+\beta_{0,10}\right) & \beta_{2,0} \\
\alpha_{2,0} & \alpha_{2,1} & 0
\end{array}\right]
$$

where $\alpha_{0,0}=\alpha_{0,0}(0)+z^{0} \alpha_{0,00}\left(z^{0}\right)$ and $\beta_{0,1}=\beta_{0,1}(0,0)+z^{0} \beta_{0,10}\left(z^{0}\right)+z^{1} \beta_{0,11}$. 
Applying the Implicit Function Theorem, if $\epsilon:=\left\|\widetilde{A}-A_{1}\right\|+\left\|\widetilde{B}-B_{1}\right\|$ is sufficiently small, there is a holomorphic matrix $h$ with $\|h\| \leq$ Const. $\epsilon$ such that $P\left((1+h) \widetilde{A}, \widetilde{B}(1+h)^{-1}\right)=(0,0,0)$. This means that $(1+h) \widetilde{A}-A_{1}$ is constant, that the third component of $\widetilde{B}(1+h)^{-1}$ is constant, and that the first component of $\widetilde{B}(1+h)^{-1}$ has the form $\beta_{0}\left(z^{0}\right)+z^{1} \beta_{01}$ where $\beta_{01}$ is constant.

Write $(1+h) \widetilde{A}=\left[\begin{array}{lll}z^{0} \mathbf{1}_{K}+\tilde{a}_{0} & z^{1} \mathbf{1}_{K}+\tilde{a}_{1} & \tilde{a}_{2}\end{array}\right]^{T}$ and $\widetilde{B}(1+h)^{-1}=\left[\begin{array}{llll}\beta_{0}+z^{1} \beta_{01} & \beta_{1} & \tilde{b}_{2}\end{array}\right]$ where $\beta_{01}, b_{2}$ are constant and $\beta_{0}$ is a function of $z^{0}$ alone. Since $1+h$ is close to 1 and the first component of $\widetilde{B}$ is close to $z^{1} \mathbf{1}_{K}$, the matrix $\beta_{01}$ is close to the identity; similarly, $\tilde{a}_{0}, \tilde{a}_{1}, \tilde{a}_{2}, \tilde{b}_{2}$ are close to 0 .

The monad equation $\widetilde{B} \widetilde{A}=0$ implies that

$$
\left(\beta_{0}+z^{1} \beta_{01}\right)\left(z^{0} \mathbf{1}_{K}+\tilde{a}_{0}\right)+\beta_{1}\left(z^{1} \mathbf{1}_{K}+\tilde{a}_{1}\right)+\tilde{b}_{2} \tilde{a}_{2}=0 ;
$$

i.e.,

$$
\left[\beta_{0}\left(z^{0} \mathbf{1}_{K}+\tilde{a}_{0}\right)+\beta_{1}\left(z^{0}, 0\right) \tilde{a}_{1}+\tilde{b}_{2} \tilde{a}_{2}\right]+z^{1}\left[\beta_{01}\left(z^{0} \mathbf{1}_{K}+\tilde{a}_{0}\right)+\beta_{1}+\beta_{1,1} \tilde{a}_{1}\right]=0 .
$$

where $\beta_{1}$ has been decomposed into $\beta_{1}\left(z^{0}, 0\right)+z^{1} \beta_{1,1}$. The first term in brackets on the left of (5.8) is a function of $z^{0}$ alone, so it follows that each of the two terms must vanish separately. The vanishing of the second term implies

$$
\left[\beta_{01}\left(z^{0} \mathbf{1}_{K}+\tilde{a}_{0}\right)+\beta_{1}\left(z^{0}, 0\right)\right]+\beta_{1,1}\left(z^{1} \mathbf{1}_{K}+\tilde{a}_{1}\right)=0 .
$$

To proceed requires a small lemma:

Lemma 5.10. Suppose $c \in G L(k, \mathbb{C}), v_{0} \in \mathbb{C}^{k}$ is a row vector, and $v_{1}$ is a row vector of holomorphic functions of the single complex variable $z$ in an open set $\Omega \subset \mathbb{C}$. If $v_{0}+v_{1}(z \mathbf{1}+c) \equiv 0$ in $\Omega$ and all eigenvalues of $c$ lie within $\Omega$ then it follows $v_{0}=0 \equiv v_{1}$.

Proof: Choose $g \in G L(k, \mathbb{C})$ such that $g^{-1} c g:=u$ is upper triangular. Then $v_{0} g+v_{1} g(z \mathbf{1}+u) \equiv 0$. The diagonal entries in $u$ are the eigenvalues of $c$ and by working successively along the the components of $v_{0} g$, $v_{1} g$, the vanishing of each component in turn follows by taking $z$ to be minus the appropriate eigenvalue.

Since $\tilde{a}_{1}$ is small, so too are its eigenvalues and therefore they can be assumed to be well within the unit disk. Hence the lemma can be applied to (5.9) (holding $z^{0}$ fixed and taking $z=z^{1}$ ) to give $\beta_{1,1}=0$ and $\beta_{1}\left(z^{0}, 0\right)=-\beta_{01}\left(z^{0} \mathbf{1}_{K}+\tilde{a}_{0}\right)$, so $\beta_{1}$ is the linear function $-\beta_{01}\left(z^{0} \mathbf{1}_{K}+\tilde{a}_{0}\right)$. Substituting this into the vanishing of the first term in (5.8) gives the equation $\beta_{0}\left(z^{0} \mathbf{1}_{K}+\tilde{a}_{0}\right)-\beta_{01}\left(z^{0} \mathbf{1}_{K}+\tilde{a}_{0}\right) \tilde{a}_{1}+\tilde{b}_{2} \tilde{a}_{2}=0$, or

$$
\left(\beta_{0}-\beta_{01} \tilde{a}_{1}\right)\left(z^{0} \mathbf{1}_{K}+\tilde{a}_{0}\right)+\tilde{b}_{2} \tilde{a}_{2}+\beta_{01}\left(\tilde{a}_{1} \tilde{a}_{0}-\tilde{a}_{0} \tilde{a}_{1}\right)=0 .
$$

Since $\tilde{a}_{0}$ is small Lemma 5.10 can be applied again to obtain $\beta_{0}=\beta_{01} \tilde{a}_{1}$ and $\tilde{b}_{2} \tilde{a}_{2}+\beta_{01}\left(\tilde{a}_{1} \tilde{a}_{0}-\tilde{a}_{0} \tilde{a}_{1}\right)=0$. Thus

$$
\widetilde{B} h^{-1}=\beta_{01}\left[\mathbf{1}_{K}+\tilde{a}_{1} \quad-\left(z^{0} \mathbf{1}_{K}+\tilde{a}_{0}\right) \quad \beta_{01}^{-1} \tilde{b}_{2}\right],
$$

which completes the proof of the proposition.

Consider now the new monad $M_{1}^{\prime}: \quad 0 \longrightarrow K(-1) \stackrel{(1+h) g A}{\longrightarrow} \stackrel{d_{W}}{W} B^{-1} \stackrel{(1+h)^{-1}}{\longrightarrow} K(1) \longrightarrow 0$ constructed from (5.4) using Proposition 5.5. As in the case of the monads $M^{\prime \prime}$ and $M^{\prime}$, the automorphism $(1+h) g$ induces an isomorphism between the cohomologies $E\left(M^{\prime \prime}\right)$ and $E\left(M_{1}^{\prime}\right)$ such that the connection on $E\left(M^{\prime \prime}\right)$ induced by hermitian projection from the flat metric on $W$ and the connection $d_{W}^{\prime \prime}$ (i.e., $d_{\rho A}$ in $B(0,2)$ ) is the "pull-back" of the connection $d_{1}^{\prime}$ on $E\left(M_{1}^{\prime}\right)$ induced by the flat metric on $W$ and the connection $d_{W}$.

Since the constant terms in the new holomorphic maps $\widetilde{A}, \widetilde{B}$ are close to $0, M_{1}^{\prime}$ can only degenerate near the origin. But by construction, it is non-degenerate in the unit polydisk, and therefore it defines a non-degenerate monad on all of $\mathbb{P}_{2}$. Thus the cohomology $E\left(M_{1}^{\prime}\right)$ is a holomorphic $r$-bundle on $\mathbb{P}_{2}$ with $c_{2}=k$ and which is trivial on $L_{\infty}$. By Theorem 0.1 of [B4] there is a smooth automorphism $g_{1}^{\prime}$ of $E\left(M_{1}^{\prime}\right)$ such that $d_{2}^{\prime}:=g_{1}^{\prime} \cdot d_{1}^{\prime}$ is anti-self-dual (with respect to the flat metric on $\mathbb{C}^{2}$ ) with $g_{1}^{\prime *} g_{1}^{\prime}=1$ on $L_{\infty}$.

Composing $g_{1}^{\prime}$ with the map on cohomology induced by $(1+\chi h) g$ for some appropriate cutoff function $\chi$ and then fixing a unitary isomorphism between $E\left(M^{\prime \prime}\right)$ and $E\left(M_{1}^{\prime}\right)$, it follows that there is a smooth automorphism $g_{2}$ of $E$ such that in $B(0,1 / 2), g_{2} \cdot \rho A$ is the restriction of an instanton on $S^{4}, \widehat{F}\left(g_{2} \cdot \rho A\right)$ is everywhere uniformly bounded in $C^{0}$, and $g_{2}^{*} g_{2}=1$ on $L_{\infty}$. Since $\triangle\left(\log \left|g_{2}\right|^{2}\right) \leq 2\left(|\widehat{F}(\rho A)|+\left|\widehat{F}\left(g_{2} \cdot \rho A\right)\right|\right) \leq$ Const. with a similar equation for $g_{2}^{-1}$, it follows from the Maximum Principle that $g_{2}$ and its inverse remain uniformly bounded in $C^{0}$ as the sequence concentrates. By Lemma 2.1 the sequence of instantons defined 
by the monads $M_{1}^{\prime}$ converges everywhere in $B(0,1 / 2)$ except at the origin, and on $B(0,1 / 2) \backslash\{0\}$ the limit $g_{2, \infty}$ gives an isomorphism between the holomorphic bundle defined by the limit of the connections $\rho A$ and the limiting instanton. Part 4 of that lemma therefore implies that the sequence of instantons defined by the monads $M_{1}^{\prime}$ is bubbling all of its curvature at the origin and degenerates nowhere else. Moreover, if the main theorem can be proved for the sequence of instantons $\left\{E\left(M_{1}^{\prime}\right)\right\}$, then because the maps $g_{2}$ and their inverses remain uniformly bounded, the lemma implies that the same convergence property is enjoyed by the sequence of connections $\{\rho A\}$.

The proof of Statement 3 of Theorem 1.3 will therefore be essentially complete if it can be shown that a sequence of instantons on $\mathbb{C}^{2}$ which is bubbling all of its charge at the origin can be made to converge by blowing up and pulling back. This is achieved in the next section.

\section{Convergence of monads.}

As stated in $\S 3$, every instanton on $S^{4}$ can be described by a monad of the form

$$
\mathbb{P}_{2}: \quad 0 \longrightarrow K(-1) \stackrel{A}{\longrightarrow} W \stackrel{B}{\longrightarrow} K(1) \longrightarrow 0 \quad,
$$

where $A=\left[\begin{array}{llll}z^{0} \mathbf{1}_{K}+a_{0} & z^{1} \mathbf{1}_{K}+a_{1} & a_{2}\end{array}\right]^{T}, B=\left[\begin{array}{lll}z^{1} \mathbf{1}_{K}+a_{1} & -\left(z^{0} \mathbf{1}_{K}+a_{0}\right) & b_{2}\end{array}\right]$ with the constants $a_{i}, b_{j}$ satisfying the monad condition $a_{1} a_{0}-a_{0} a_{1}+b_{2} a_{2}=0$ and the instanton condition $a_{0} a_{0}^{*}-a_{0}^{*} a_{0}+a_{1} a_{1}^{*}-$ $a_{1}^{*} a_{1}+b_{2} b_{2}^{*}-a_{2}^{*} a_{2}=0$. The monad is the restriction to $\mathbb{P}_{2}$ of a monad on $\mathbb{P}_{3}$ of the form (6.1), $\mathbb{P}_{3}$ being the twistor space for $S^{4}$ ([AHS]).

There is an analogous description of (self-dual) instantons on $\mathbb{C P}_{2}$ in terms of monads on the blowup $\widetilde{\mathbb{P}}_{2}$ of $\mathbb{P}_{2}$ at a point. The twistor space for $\mathbb{C P}_{2}$ is the flag manifold $\mathbb{F}=\left\{(Z, W) \in \mathbb{P}_{2} \times \mathbb{P}_{2}^{*} \mid Z \cdot W=0\right\}$ where $Z=\left(Z^{0}, Z^{1}, Z^{2}\right), W=\left(W_{0}, W_{1}, W_{2}\right)$ are homogeneous coordinates. The hypersurface $W_{2}=0$ is isomorphic the blowup $\widetilde{\mathbb{P}}_{2}$ of $\mathbb{P}_{2}$ at $(0,0,1)$, realised by the projection on the first factor, and is also isomorphic to the the Hirzebruch surface $H_{1}$, realised as a $\mathbb{P}_{1}$ bundle over $\mathbb{P}_{1}$ by projection onto second factor. On this hypersurface, the equation $Z \cdot W=0$ implies that $\left(Z^{0}, Z^{1}\right)=\lambda\left(-W_{1}, W_{0}\right)$ for some section $\lambda$ of $\mathcal{O}(1,-1)$, this section defining the exceptional line.

Holomorphic bundles on $H_{1}$ were studied in [B2]. With $x:=c_{1}(\mathcal{O}(1,0))$ and $y:=c_{1}(\mathcal{O}(0,1))$ being first Chern classes of the the pull-backs of the Hopf line bundles, a holomorphic $r$-bundle $E$ on $\widetilde{\mathbb{P}}_{2}$ with $c_{1}(E)=l(x-y)$ and $c_{2}(E)=k x y$ which is trivial on $L_{\infty}=\left\{Z^{2}=0=W_{2}\right\}$ is given by a monad of the form

$$
\widetilde{\mathbb{P}}_{2}: \quad 0 \longrightarrow K_{1}(0,-1) \stackrel{(a, b)}{\longrightarrow} N \oplus K_{2}(1,-1) \stackrel{(c, d)}{\longrightarrow} K_{3}(1,0) \longrightarrow 0
$$

where $K_{1}, K_{2}, K_{3}$ and $N$ are complex vector spaces of dimension $k+\frac{1}{2} l(l-1), k+\frac{1}{2} l(l+1), k+\frac{1}{2} l(l-1)$ and $r+k+\frac{1}{2} l(l-3)$ respectively. The results of [B4], or alternatively the direct results of King [Ki] when $c_{1}(E)=0$, imply that each such monad can be extended to a unitary monad on $\mathbb{F}$, these unitary monads being described in [B1]. That is, the monad (6.2) is isomorphic to a monad of the same form on $\mathbb{F}$ for which the map $(c, d)$ is

$$
(c(Z), d(W))=\left(a(\bar{Z})^{*},-b(\bar{W})^{*}\right) .
$$

Again, the failure of the map $(a(W), b(Z))$ to be injective at some point $(Z, W)$ corresponds precisely to the singularity of the corresponding self-dual connection at the point $[\bar{Z} \times W] \in \mathbb{C P}_{2}$; for details, see [B1].

Given a monad on $\widetilde{\mathbb{P}}_{2}$ of the form (6.2), a monad on $\mathbb{P}_{2}$ of the form (6.1) is constructed by taking direct images, the latter monad being non-singular iff $b(0,0,1)$ is an isomorphism (and the original is non-singular). Of more interest here is the construction of a monad on $\widetilde{\mathbb{P}}_{2}$ from one on $\mathbb{P}_{2}$. Given a monad on $\mathbb{P}_{2}$ of the form (6.1) with cohomology $E$, construct a monad on $\widetilde{\mathbb{P}}_{2}$ of the form (6.2) corresponding to $\pi^{*} E$ as follows: set $K_{1}:=K=: K_{3}, K_{2}:=\operatorname{Im} A_{2}$ where $A_{2}:=A(0,0,1)$ and $N:=K_{2}^{\perp}$. Let $\Pi$ • denote hermitian projection, and set $a(W):=\Pi_{N} A\left(-W_{1}, W_{0}, 0\right), b(Z):=\Pi_{K_{2}} A(Z), c(Z)=\left.B(Z)\right|_{N}$ and $d(W):=\left.B\left(-W_{1}, W_{0}, 0\right)\right|_{K_{2}}$.

The unitary condition (6.3) is not satisfied in general, but it is possible to write down, in a relatively explicit way, the isomorphic monad on $\widetilde{\mathbb{P}}_{2}$ which does satisfy the unitary condition and possesses the same holomorphic trivialisation on $L_{\infty}$. Given the monad $(6.1)$, let $a(W), b(Z)$ be as constructed in the preceding paragraph. The new maps $a^{\prime}(W), b^{\prime}(Z), c^{\prime}(Z), d^{\prime}(W)$ are then given by

$$
\left[\begin{array}{l}
a^{\prime} \\
b^{\prime}
\end{array}\right]=\left[\begin{array}{cc}
\phi_{N} & 0 \\
\lambda \phi_{K N} & \phi_{K}
\end{array}\right]\left[\begin{array}{l}
a \\
b
\end{array}\right] g_{1}^{-1}, \quad\left[\begin{array}{cc}
c^{\prime} & d^{\prime}
\end{array}\right]=g_{2}\left[\begin{array}{ll}
c & d
\end{array}\right]\left[\begin{array}{cc}
\phi_{N} & 0 \\
\lambda \phi_{K N} & \phi_{K}
\end{array}\right]^{-1}
$$


for some $\phi_{N} \in G L(N), \phi_{K} \in G L\left(K_{2}\right), \phi_{K N} \in \operatorname{Hom}\left(N, K_{2}\right), g_{1} \in G L\left(K_{1}\right)$ and $g_{2} \in G L\left(K_{1}\right)$. Setting $\phi:=\left[\begin{array}{cc}\phi_{N} & 0 \\ \phi_{K N} & \phi_{K}\end{array}\right] \in G L(W)$, this is

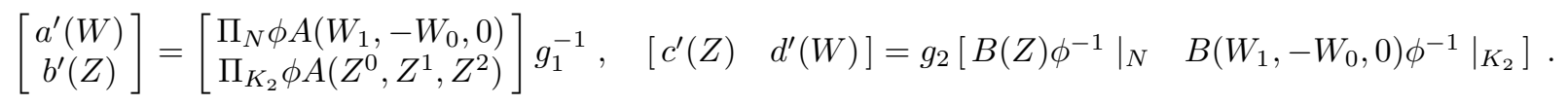

The requirement that the cohomologies of the two monads have the same trivialisations on $L_{\infty}$ is equivalent to the condition that $\phi$ be the identity on the subspace $R \subset W$, which implies that in terms of the decomposition $W=K \oplus K \oplus R$ it should have the form

$$
\phi=\left[\begin{array}{lll}
\phi_{00} & \phi_{01} & 0 \\
\phi_{10} & \phi_{11} & 0 \\
\phi_{20} & \phi_{21} & 1
\end{array}\right]
$$

Restricting to $L_{\infty}$, the unitary condition (6.3) on the new monad is

$$
\left[\begin{array}{c}
\Pi_{N} \phi A\left(W_{1},-W_{0}, 0\right) g_{1}^{-1} \\
\Pi_{K_{2}} \phi A\left(Z^{0}, Z^{1}, 0\right) g_{1}^{-1}
\end{array}\right]=\left[\begin{array}{c}
\left(\left.g_{2} B\left(\bar{W}_{0}, \bar{W}_{1}, 0\right) \phi^{-1}\right|_{N}\right)^{*} \\
-\left(g_{2} B\left(\bar{Z}^{1},-\bar{Z}^{0}, 0\right) \phi^{-1}||_{K_{2}}\right)^{*}
\end{array}\right]
$$

that is, $\phi^{*} \phi A\left(Z^{0}, Z^{1}, 0\right)=B\left(-\bar{Z}^{1}, \bar{Z}^{0}, 0\right)^{*} g_{2}^{*} g_{1}$, implying $\phi^{*} \phi=\left[\begin{array}{ccc}g_{2}^{*} g_{1} & 0 & * \\ 0 & g_{2}^{*} g_{1} & * \\ 0 & 0 & *\end{array}\right]$. Using (6.4), it follows that there is a unitary matrix $U \in G L(K \oplus K)$ together with a positive definite matrix $P \in G L(K)$ such that

$$
\phi=\left[\begin{array}{ccc}
U_{00} P^{1 / 2} & U_{01} P^{1 / 2} & 0 \\
U_{10} P^{1 / 2} & U_{11} P^{1 / 2} & 0 \\
0 & 0 & 1
\end{array}\right] \quad \text { and } \quad g_{2}^{*} g_{1}=P .
$$

The condition that $\phi$ should preserve the subspace $K_{2}=\operatorname{Im} A_{2}$ for $A_{2}:=A(0,0,1)$ means that $\phi A_{2}=$ $A_{2} \chi$ for some $\chi \in G L(K) ; \chi$ must be $\psi_{0}^{-1} A_{2}^{*} \phi A_{2}$ for $\psi_{0}:=A_{2}^{*} A_{2}$. The unitary condition (6.3) implies one more constraint on the matrices $\phi, g_{1}, g_{2}$, namely that if $a^{\prime}\left(0,0, W_{2}\right):=c^{\prime}\left(0,0, \bar{W}_{2}\right)^{*}$ and $d^{\prime}\left(0,0, W_{2}\right):=$ $-b^{\prime}\left(0,0, \bar{W}_{2}\right)^{*}$ then $c^{\prime}(0,0,1) a^{\prime}(0,0,1)+d^{\prime}(0,0,1) b^{\prime}(0,0,1)=c^{\prime}(1,0,0) a^{\prime}(1,0,0)+d^{\prime}(1,0,0) b^{\prime}(1,0,0)$. A straightforward calculation shows that this condition is equivalent to

$$
a_{0} P^{-1} a_{0}^{*}+a_{1} P^{-1} a_{1}^{*}+a_{0} Q^{-1} a_{0}^{*}+a_{1} Q^{-1} a_{1}^{*}+b_{2} b_{2}^{*}=P^{-1}+P^{-1} Q P^{-1}
$$

for $Q:=\chi^{*} \psi_{0} \chi$.

Note that the equation $\phi A_{2}=A_{2} \chi$ implies $\phi^{*} \phi A_{2}=\phi^{*} A_{2} \chi$, so $\chi^{*} A_{2}^{*} \phi=A_{2}^{*} \phi^{*} \phi$. Hence $\chi^{*} \psi_{0} \chi=$ $\chi^{*} A_{2}^{*} \phi A_{2}=A_{2}^{*} \phi^{*} \phi A_{2}$, or

$$
(Q=) \quad \chi^{*}\left(a_{0}^{*} a_{0}+a_{1}^{*} a_{1}+a_{2}^{*} a_{2}\right) \chi=a_{0}^{*} P a_{0}+a_{1}^{*} P a_{1}+a_{2}^{*} a_{2} .
$$

(In fact, the condition $\phi A_{2}=A_{2} \chi$ and the form of $\phi$ imply $a_{2}=a_{2} \chi$, so the terms involving $a_{2}$ can be dropped from this equation).

Suppose now that the monad (6.1) is a typical element in a sequence of such (satisfying the instanton condition) such that $a_{0}, a_{1}, a_{2}, b_{2}$ are converging to 0 . The monads on $\widetilde{\mathbb{P}}_{2}$ constructed above (satisfying the unitary condition) then correspond to a sequence of self-dual instantons on $\mathbb{C P}_{2}$. By weak compactness, after gauge transformations, there is a subsequence of these connections together with a finite set of points where the curvatures concentrate, converging smoothly off this set of points. In terms of the monads, this means that there are unitary automorphisms $U_{N}, U_{K}, U_{K_{2}}$ such that a subsequence of $\left(U_{N} a^{\prime} U_{K}^{-1}, U_{K_{2}} b^{\prime} U_{K}^{-1}\right)$ converges to define a monad on $\widetilde{\mathbb{P}}_{2}$ which is degenerate only at a finite set of points. Thus if $U_{W}:=\left[\begin{array}{cc}U_{N} & 0 \\ 0 & U_{K_{2}}\end{array}\right]$, then a subsequence of the maps $U_{W} \phi A(Z) U_{K}^{-1}$ converges to define a monad which is degenerate at only finitely many points in $\widetilde{\mathbb{P}}_{2}$. By passing to a subsequence it can be assumed that the unitary maps $U, U_{W}, U_{K}$ converge.

Restricting now to a point on $L_{\infty}$ which is not a point of degeneration, it follows that the automorphisms $P^{1 / 2} g_{1}^{-1}$ converge in $G L(K)$, so in fact there is no degeneration on $L_{\infty}$. Restricting next to a point in $\mathbb{P}_{2} \backslash\left\{L_{\infty} \cup\{(0,0,1)\}\right\}$ which is not a point of degeneration, it follows that the maps $P^{1 / 2} a_{0} g_{1}^{-1}, P^{1 / 2} a_{1} g_{1}^{-1}$ 
and $a_{2} g_{1}^{-1}$ converge. A point $\left(z^{0}, z^{1}\right) \in \mathbb{C}^{2} \backslash\{0\}$ is a point of degeneration if there is a non-zero vector $v \in K$ such that $\lim \left(P^{1 / 2} g_{1}^{-1} z^{0}+P^{1 / 2} a_{0} g_{1}^{-1}\right) v \lim \left(P^{1 / 2} g_{1}^{-1} z^{1}+P^{1 / 2} a_{1} g_{1}^{-1}\right) v$ and $\lim a_{2} g_{1}^{-1} v$ all vanish. But since $\operatorname{det}\left(P^{1 / 2} g_{1}^{-1} z^{0}+P^{1 / 2} a_{0} g_{1}^{-1}\right)=\operatorname{det}\left(P^{1 / 2} g_{1}^{-1}\right) \operatorname{det}\left(z^{0} \mathbf{1}_{K}+g_{1} a_{0} g_{1}^{-1}\right)=\operatorname{det}\left(P^{1 / 2} g_{1}^{-1}\right) \operatorname{det}\left(z^{0} \mathbf{1}_{K}+a_{0}\right)$ which converges to $\operatorname{det}\left(P^{1 / 2} g_{1}^{-1}\right)\left(z^{0}\right)^{k}$, with a similar statement for $a_{1}$, it follows that there are in fact no points of degeneration in $\mathbb{C}^{2} \backslash\{0\}$. Thus if the sequence of monads on $\widetilde{\mathbb{P}}_{2}$ is to degenerate, it can do so only at points of the exceptional line.

Now let $\tilde{a}_{0}:=P^{1 / 2} a_{0}\left(\chi^{*} \psi_{0} \chi\right)^{-1 / 2}, \tilde{a}_{1}:=P^{1 / 2} a_{1}\left(\chi^{*} \psi_{0} \chi\right)^{-1 / 2}, \tilde{a}_{2}:=a_{2}\left(\chi^{*} \psi_{0} \chi\right)^{-1 / 2}$ so $\tilde{a}_{0}^{*} \tilde{a}_{0}+\tilde{a}_{1}^{*} \tilde{a}_{1}+$ $\tilde{a}_{2}^{*} \tilde{a}_{2}=\left(\chi^{*} \psi_{0} \chi\right)^{-1 / 2}\left[a_{0}^{*} P a_{0}+a_{1}^{*} P a_{1}+a_{2}^{*} a_{2}\right]\left(\chi^{*} \psi_{0} \chi\right)^{-1 / 2}=\mathbf{1}_{K}$ by (6.6). Passing to a subsequence, it can be assumed that the maps $\tilde{a}_{i}$ all converge, and the limit must satisfy the same identity.

Suppose that the sequence of monads on $\widetilde{\mathbb{P}}_{2}$ is bubbling all of its charge at a single point on the exceptional line. For the sake of argument, let this point be the point with $Z=(0,0,1), W=(0,1,0)$. Then if $A_{0}:=A(1,0,0)$ the conditions that $a^{\prime}(0,1,0), b^{\prime}(0,0,1) \rightarrow 0$ are

$$
\phi A_{0} g_{1}^{-1}-A_{2} \psi_{0}^{-1} A_{2}^{*} \phi A_{0} g_{1}^{-1} \rightarrow 0, \quad \psi_{0}^{-1 / 2} A_{2}^{*} \phi A_{2} g_{1}^{-1} \rightarrow 0 .
$$

Since $A_{2}^{*} \phi=\chi^{*-1} A_{2}^{*}\left(\phi^{*} \phi\right)$ it follows that $A_{2}^{*} \phi A_{0} g_{1}^{-1}=\chi^{*-1} A_{2}^{*}\left(\phi^{*} \phi\right) A_{0} g_{1}^{-1}=\chi^{*-1} a_{0}^{*} P g_{1}^{-1}$, and since $U^{-1}\left[\begin{array}{l}a_{0} \\ a_{1}\end{array}\right]=\left[\begin{array}{l}P^{1 / 2} a_{0} \\ P^{1 / 2} a_{1}\end{array}\right] \chi^{-1}$, multiplying the first equation in (6.7) through by the inverse of $\left[\begin{array}{cc}U & 0 \\ 0 & 1\end{array}\right]$ implies

$$
\left[\begin{array}{c}
P^{1 / 2} g_{1}^{-1} \\
0 \\
0
\end{array}\right]-\left[\begin{array}{c}
P^{1 / 2} a_{0} \\
P^{1 / 2} a_{1} \\
a_{2}
\end{array}\right]\left(\chi^{*} \psi_{0} \chi\right)^{-1}\left[\begin{array}{lll}
a_{0}^{*} P & a_{1}^{*} P & a_{2}^{*}
\end{array}\right]\left[\begin{array}{c}
1 \\
0 \\
0
\end{array}\right] g_{1}^{-1} \rightarrow 0
$$

or

$$
\left[\begin{array}{c}
P^{1 / 2} g_{1}^{-1}-\tilde{a}_{0} \tilde{a}_{0}^{*} P^{1 / 2} g_{1}^{-1} \\
-\tilde{a}_{1} \tilde{a}_{0}^{*} P^{1 / 2} g_{1}^{-1} \\
-\tilde{a}_{2} \tilde{a}_{0}^{*} P^{1 / 2} g_{1}^{-1}
\end{array}\right] \rightarrow 0 .
$$

Finally, since $P^{1 / 2} g_{1}^{-1}$ is converging to a non-singular automorphism of $K$, multiplying on the right by the inverse of $P^{1 / 2} g_{1}^{-1}$ shows that the first equation in (6.7) is equivalent to

$$
\left[\begin{array}{c}
\mathbf{1}_{K}-\tilde{a}_{0} \tilde{a}_{0}^{*} \\
-\tilde{a}_{1} \tilde{a}_{0}^{*} \\
-\tilde{a}_{2} \tilde{a}_{0}^{*}
\end{array}\right] \rightarrow 0
$$

(the second condition is equivalent to $\psi_{0}^{1 / 2} \chi g_{1}^{-1} \rightarrow 0$, but this is not required here). Thus $\tilde{a}_{0}$ converges to an element of $U(K)$ and $\tilde{a}_{1}, \tilde{a}_{2}$ converge to 0 .

In general, if the sequence is bubbling all of its charge at a point on the exceptional line where $\left[W_{0}, W_{1}, 0\right]=\left[w_{0}, w_{1}, 0\right]$ for $\left|w_{0}\right|^{2}+\left|w_{1}\right|^{2}=1$, then it follows that $\tilde{a}_{2} \rightarrow 0$ and $\left(\tilde{a}_{0}, \tilde{a}_{1}\right) \rightarrow\left(\tilde{a} w_{1},-\tilde{a} w_{0}\right)$ for some $\tilde{a} \in U(K)$.

Recall that $\tilde{a}_{i}=P^{1 / 2} a_{i}\left(\chi^{*} \psi_{0} \chi\right)^{-1 / 2}$ for $i=0,1$. Thus if each term in the original sequence on $\mathbb{P}_{2}$ is such that $\operatorname{det} a_{0}=0=\operatorname{det} a_{1}$ then it is not possible for the corresponding sequence of monads on $\widetilde{\mathbb{P}}_{2}$ to bubble all of its charge at a single point. (In particular, if $k=1$, then the corresponding sequence on $\widetilde{\mathbb{P}}_{2}$ must converge.) If $-\lambda_{i}$ is an eigenvalue of $a_{i}$ and the original sequence of instantons defined by (6.1) is pulled back under the "translation" $\left(Z^{0}, Z^{1}, Z^{2}\right) \mapsto\left(Z^{0}+\lambda_{0} Z^{2}, Z^{1}+\lambda_{1} Z^{2}, Z^{2}\right)$ (i.e., the isometry of the flat metric on $\left.\mathbb{C}^{2}\left(z^{0}, z^{1}\right) \mapsto\left(z^{0}+\lambda_{0}, z^{1}+\lambda_{1}\right)\right)$, then $\left(a_{0}, a_{1}, a_{2}, b_{2}\right) \mapsto\left(a_{0}+\lambda_{0} \mathbf{1}, a_{1}+\lambda_{1} \mathbf{1}, a_{2}, b_{2}\right)$, and the sequence of monads on $\widetilde{\mathbb{P}}_{2}$ corresponding to this "translated" sequence could not bubble all of its charge at a single point.

Instead of pulling back the monads on $\mathbb{P}_{2}$ under these "translations" and then constructing corresponding monads on $\widetilde{\mathbb{P}}_{2}$, the latter monads can be viewed as those which are obtained by blowing up at the points $\left(-\lambda_{0},-\lambda_{1}, 1\right) \in \mathbb{P}_{2}$ and constructing the corresponding monads for each of these blowups, then switching to a fixed set of coordinates. Iterating the entire procedure at most $2 k-1$ times, it follows that after blowing up at a suitable (convergent) set of points, there is a convergent subsequence of the corresponding sequence of Hermitian-Einstein connections.

Thus, if $\omega_{0}$ is the standard flat metric on $\mathbb{C}^{2}$, there is a converging family of blowups of $\mathbb{C}^{2}$ converging to a blowup $\widetilde{\mathbb{C}}^{2} \stackrel{\pi}{\rightarrow} \mathbb{C}^{2}$ with exceptional divisor $\pi^{-1}(0)$, together with automorphisms $g_{i_{j}}$ satisfying $g_{i_{j}}^{*} g_{i_{j}}=\mathbf{1}$ on $L_{\infty}$ and $\sup _{K}\left(\left|g_{i_{j}}\right|+\left|g_{i_{j}}^{-1}\right|\right) \leq C_{K}$ for each compact set $K \subset \mathbb{C}^{2} \backslash\{0\}$ such that $\left\{g_{i_{j}} \cdot \pi_{i_{j}}^{*} \rho A_{i_{j}}\right\}$ converges strongly on $\widetilde{\mathbb{C}}^{2}$; moreover, for some metric of the form $\omega_{0 \alpha}$, the connections satisfy $\omega_{0 \alpha} \wedge F\left(g_{i_{j}} \cdot \pi_{i_{j}}^{*} \rho A_{i_{j}}\right)=0$. 
Note that if $\omega_{\beta}$ is any other metric on $\widetilde{\mathbb{C}}^{2}$ which is close to $\omega_{0}$ in a neighbourhood of $L_{\infty}$ in the sense of [B3], it follows from the main result of that reference together with the Maximum Principle and Lemma 2.1 that the corresponding sequence of $\omega_{\beta}$-Hermitian-Einstein connections has a strongly convergent subsequence.

To complete the proof of Theorem 1.3, let $X, \omega,\left\{A_{i}\right\}$ be as given in the hypotheses with $A_{i}$ converging weakly off $S \subset X$ to define a quasi-bundle $E$. Assume for the moment that $E$ is stable, and replace $\omega$ by a metric which is standard in a neighbourhood of each of the points of $S$ and $A_{i}$ by the Hermitian-Einstein connection associated to the new metric, placed in a gauge as specified by Lemma 2.1. Using the procedure of $\S \S 1-6$ and passing to a subsequence if necessary, construct a converging sequence of blowups $\widetilde{X}_{i}$ of $X$ consisting of at most $2 C\left(E_{\text {top }}\right)-2 C(E)-1$ individual blowups converging to a blowup $\widetilde{X}$ of $X$ so that in a neighbourhood of each component of the exceptional divisor there are complex automorphisms $g_{i}$ with $\left|g_{i}\right|+\left|g_{i}^{-1}\right|$ uniformly bounded on compact subsets of the complement of this component and with $g_{i} \cdot \pi_{i}^{*} A_{i}$ converging strongly. Replacing $g_{i}$ by $\exp \left(\frac{1}{2} \rho \log \left(g_{i}^{*} g_{i}\right)\right)$ for some smooth cutoff function $\rho$ and making a gauge transformation then extends $g_{i}$ to the whole of $\widetilde{X}_{i}$ so that it is the identity on the complement of a neighbourhood of the exceptional divisor. Thus, using Proposition 1.2 with Lemmas 2.1 and 2.2, the proofs of Statements 1-4 of Theorem 1.3 are complete.

The proof of Statement 6 of Theorem 1.3 is exactly the same argument as that which followed the proof of Lemma 2.2, once it is known that each bundle in the sequence of pulled-back bundles is stable with respect to the same metric (or rather, its class in $H^{2}(\widetilde{X}, \mathbb{R})$ ). If $b_{1}(X)$ is even, this follows immediately from Proposition 1.2, but in general, if it were not true, then it would be possible to construct a sequence of stable bundles on $X$ converging weakly to a bundle which destabilises the weak limit $E$; (for details, see [B5, Para. $1 \S 6]$.

To deal with the case of weak limits which are not stable, there is a useful technique for "stabilising" semi-stable $r$-bundles $E$. Pick any $x_{0} \in X$ and blow up $X$ at this point. Let $\mathcal{O}(1)$ be the dual of the line bundle defining the exceptional divisor $L_{0}$, and in an annular region surrounding $L_{0}$, identify $E$ with $\mathcal{O}(1) \oplus \mathcal{O}^{r-1}$ This defines a bundle $\widetilde{E}$ on $\widetilde{X}$ restricting to $\mathcal{O}(1) \oplus \mathcal{O}^{r-1}$ on $L_{0}$ with $\left(\pi_{*} \widetilde{E}\right)^{* *}=E$ and $C(\widetilde{E})=C(E)+(r-1) / 2 r$. It is not hard to show that the isomorphism classes of such bundles can be identified with $\mathbb{P}\left(E_{x_{0}}^{*}\right)$; see [B5]. The following pair of results is also proved in that reference (Prop. 4.2, Lemma 5.1):

Lemma 6.8. If the above operation is applied at any $n>r^{2}$ distinct points in $X$, then for generic choices of the elements of $\mathbb{P}\left(E_{x_{i}}^{*}\right)$ the resulting bundle $\widetilde{E}$ on $\widetilde{X}$ is $\omega_{\epsilon \alpha_{0}}$-stable for all $\epsilon$ sufficiently small, where $\alpha_{0}=(1,1, \ldots, 1)$.

(Note: If $r=2$, then at most 3 points are required.)

Lemma 6.9. Let $\left\{A_{i}\right\}$ be a sequence of irreducible Hermitian-Einstein connections converging weakly to $A$ off $S \subset X$, and let $E_{i}$ be the corresponding stable bundle. Let $E$ be the semi-stable weak limit corresponding to $A$, and let $\widetilde{E}$ on $\widetilde{X}$ be a stabilisation of $E$ as described above with none of the blown up points lying in $S$. Then there are stabilisations $\widetilde{E}_{i}$ of $E_{i}$ (stable with respect to $\omega_{\epsilon \alpha_{0}}$ for fixed $\epsilon>0$ sufficiently small) converging weakly to $\widetilde{E}$ off $\pi^{-1}(S)$ such that if $\widetilde{A}_{i}$ is the corresponding $\omega_{\epsilon \alpha_{0}}$-Hermitian-Einstein connection, then for some complex automorphism $g_{i}$ of $E_{\mathrm{top}}, \widetilde{A}_{i}=g_{i} \cdot A_{i}$ on the complement of the exceptional divisor and $\sup _{K}\left(\left|g_{i}\right|+\left|g_{i}^{-1}\right|\right)$ is uniformly bounded for every compact subset $K$ of this complement.

Statement 5 of Theorem 1.3 follows immediately from these lemmas, completing the proof of that theorem.

\section{Bundles of rank 2.}

Suppose now that $b_{1}(X)$ is even, and let $\left\{E_{i}\right\}$ be a sequence of stable $r$-bundles all topologically isomorphic to $E_{\text {top }}$ converging weakly to $E$ off $S \subset X$. Let $\left\{\widetilde{X}_{i}\right\}$ be any sequence of blowups of $X$ converging to a blowup $\tilde{X} \stackrel{\pi}{\rightarrow} X$ of $X$, equipped with positive $\bar{\partial} \partial$-closed $(1,1)$-forms ${\underset{\sim}{i}}_{i}^{*} \omega+\rho_{\alpha}$ for suitable $\alpha \in \mathbb{R}^{n}$ satisfying $|\alpha|<\epsilon_{0}$ where $\epsilon_{0}$ is as in Proposition 1.2. Although the blowups $\tilde{X}_{i}$ may be changing, the usual weak compactness applies, as does semi-continuity of cohomology (since a set with strictly pseudo-convex boundary will have strictly pseudo-convex boundary with respect to all nearby complex structures). Thus 
there is a finite subset $\widetilde{S} \subset \widetilde{X}$ such that (a subsequence of) $\left\{\pi^{*} E_{i}\right\}$ converges weakly to a quasi-stable limit $\widetilde{E}$ off $\widetilde{S}$ with respect to $\omega_{\alpha}$. Then $\operatorname{det} \widetilde{E}=\pi^{*} \operatorname{det} E$, and there are non-zero homomorphisms $\left(\pi_{*} \widetilde{E}\right)^{* *} \rightarrow E$, $E \rightarrow\left(\pi_{*} \widetilde{E}\right)^{* *}$.

For each $\epsilon \in(0,1]$ the same argument using the metric $\pi_{i}^{*} \omega+\rho_{\epsilon \alpha}$ yields a weak limit $\widetilde{E}_{\epsilon}$. There are only finitely many possible first Chern classes for the summands of each $\widetilde{E}_{\epsilon}$, so for sufficiently small $\epsilon$, the topological type of the summands of $\widetilde{E}_{\epsilon}$ will be independent of $\epsilon$. Since the direct image of each summand is semi-stable (by Proposition 1.2) and each must have the same have the same slope with respect to $\omega, \pi_{*} \widetilde{E}_{\epsilon}$ is semi-stable for $\epsilon$ sufficiently small.

If $E$ is stable then the map $E \rightarrow\left(\pi_{*} \widetilde{E}_{\epsilon}\right)^{* *}$ must be an isomorphism, so by Proposition 1.2, $\widetilde{E}_{\epsilon}$ is stable with respect to $\omega_{\epsilon^{\prime} \alpha}$ for all $\epsilon^{\prime} \in\left(0, \epsilon_{0} /|\alpha|\right)$. Semi-continuity of cohomology also gives a non-zero homomorphism $\widetilde{E} \rightarrow \widetilde{E}_{\epsilon}$, but since the latter is $\omega_{\alpha}$-stable, this must be an isomorphism. (Note that, regardless of the stability of $E$, if $\widetilde{E}_{\epsilon}$ is stable for some $\epsilon$ then the weak limit is independent of $\epsilon \in\left(0, \epsilon_{0} /|\alpha|\right)$.) Thus $\left(\pi_{*} \widetilde{E}\right)^{* *}=E$, and there are inter-twining operators $g_{i}$ linking the Hermitian-Einstein connections on $E_{i}$ and $\pi_{i}^{*} E_{i}$ over the complement of a neighbourhood of the exceptional divisor which, together with their inverses are uniformly bounded in $C^{0}$, and by Lemma 2.1 of [B4], $\widetilde{S} \subset \pi^{-1}(S)$; this proves the claim made in the discussion following Proposition 1.2. Note that Proposition 1.2 combined with the same argument shows that the weak limit $\widetilde{E}$ is independent of the choice of suitable $\alpha \in \mathbb{R}^{n}$ satisfying $|\alpha|<\epsilon_{0}$.

Suppose now that $E$ is only semi-stable; the situation is considerably simpler if it is assumed from now on that the rank of $E$ is 2. Thus $E=L_{1} \oplus L_{2}$ for some line bundles $L_{1}, L_{2}$ on $X$ such that $c_{1}\left(L_{1}\right)+c_{1}\left(L_{2}\right)=$ $c_{1}\left(E_{\text {top }}\right)$ and $\operatorname{deg}\left(L_{1}\right)=\operatorname{deg}\left(L_{2}\right)=\operatorname{deg}\left(E_{\text {top }}\right) / 2$.

As noted above, if $\widetilde{E}_{\epsilon}$ is stable for some $\epsilon$, then it is in fact independent of $\epsilon$. If $\widetilde{E}_{\epsilon}$ is not stable, then for $\epsilon$ sufficiently small as above it must have the form $\pi^{*} L_{1} \otimes L^{*} \oplus \pi^{*} L_{2} \otimes L$ where $L$ is a line bundle on $\widetilde{X}$ which is trivial off the exceptional divisor and satisfies $\rho_{\alpha} \cdot c_{1}(L)=0$. If $\epsilon^{\prime} \in\left(0, \epsilon_{0} /|\alpha|\right)$ and $\widetilde{E}\left(\epsilon^{\prime}\right)$ splits as $\widetilde{L}_{1} \oplus \widetilde{L}_{2}$ then by definition of the number $\epsilon_{0}$ and the condition that $\omega_{\alpha} \cdot c_{1}\left(\widetilde{L}_{1}\right)=\omega_{\alpha} \cdot c_{1}(\widetilde{E}) / 2=\omega \cdot c_{1}(E) / 2=\omega \cdot c_{1}\left(L_{1}\right)$ it must be the case that $\left(\pi_{*} \widetilde{L}_{1}\right)^{* *}=L_{1}$ (after renumbering if necessary). This implies $\widetilde{L}_{1} \otimes \pi^{*} L_{1}{ }^{*}$ is trivial off the exceptional divisor and has degree 0 with respect to $\omega_{\alpha}$. Semi-continuity of cohomology gives a non-zero section of $\widetilde{L}_{1} \otimes \pi^{*} L_{1}{ }^{*} \otimes L$, but since this line bundle has degree 0 it follows that it is trivial. Thus the weak limit $\widetilde{E}_{\epsilon}=\pi^{*} L_{1} \otimes L^{*} \oplus \pi^{*} L_{2} \otimes L$ is independent of $\epsilon \in\left(0, \epsilon_{0} /|\alpha|\right)$, isomorphic to $\widetilde{E}=\widetilde{E}_{1}$ and $\pi_{*} \widetilde{E}$ is, as before, semi-stable.

For generic choices of $\alpha$, there are no non-trivial line bundles $L$ on $\widetilde{X}$ which are trivial off the exceptional divisor and have degree 0 with respect to $\omega_{\alpha}$. For such $\alpha$, it follows that the weak limit on $\widetilde{X}$ is either $\omega_{\alpha}$-stable or is isomorphic to $\pi^{*} E$. However, even in the case of non-generic $\alpha$, if $L$ is non-trivial then $-c_{1}(L)^{2}>0$, implying $C(\widetilde{E})>C(E)$ and that the amount of charge bubbled by the sequence on the blowup is strictly less than that which was bubbled by the sequence on $X$.

With these preliminary remarks in hand, the proof of Theorem 1.4 can now be given; the method is by induction on the amount of charge bubbled by the sequence $\left\{E_{i}\right\}$.

For $E_{i}$ and $E=L_{1} \oplus L_{2}$ as above, pick 3 points $T \subset X \backslash S$ and stabilise $E$ to $E^{\prime}$ on some blowup $X^{\prime}$ of $X$ centered at $T$. Let $\alpha=\epsilon_{1}(1,1,1)$ be such that $E^{\prime}$ is $\omega_{\epsilon \alpha}$-stable for all $\epsilon \in(0,1]$, and choose stabilisations $E_{i}^{\prime}$ on $X^{\prime}$ converging weakly to $E^{\prime}$ off $S$ with respect to $\omega_{\alpha}$, as prescribed by Lemma 7.3. Now use Theorem 1.3 to construct a sequence of blowups $\widetilde{X}_{i}^{\prime}$ of $X^{\prime}$ converging to a blowup $\widetilde{X}^{\prime}$ of $X^{\prime}$ centered at $S$ with a strongly converging (sub)-sequence $\left\{\pi_{i}^{*} E_{i}^{\prime}\right\}$, stable with respect to $\pi_{i}^{*} \omega_{\alpha}+\rho_{\beta}$ for some fixed $\beta$ sufficiently small. Without loss of generality it can be assumed that $\operatorname{supp}\left(\rho_{\alpha}\right) \cap \operatorname{supp}\left(\rho_{\beta}\right)=\emptyset$, so that $\omega_{\epsilon \alpha, \delta \beta}=\pi^{\prime *} \pi^{*} \omega+\epsilon \rho_{\alpha}+\delta \rho_{\beta}$ is a positive $(1,1)$-form on $\widetilde{X}^{\prime}$ for any $(\epsilon, \delta) \in(0,1] \times(0,1]$.

Let $\widetilde{E}$ be the weak limit of the sequence $\left\{\pi^{*} E_{i}\right\}$ on $\widetilde{X}$, the latter space being that which obtained by blowing down the components $T^{\prime}$ of the exceptional divisor lying over $T$; let $\pi^{\prime}: \widetilde{X}^{\prime} \rightarrow \widetilde{X}$ be the blowing-down map. By the discussion above, it can be assumed without loss of generality that $\widetilde{E}=\pi^{*} E$; similarly, it can also be assumed without loss of generality that the weak limit of the sequence $\left\{\pi^{\prime *} \pi^{*} E_{i}\right\}$ on $\widetilde{X}^{\prime}$ is $\pi^{\prime *} \pi^{*} E$.

For each $i$ there is a map $E_{i}^{\prime} \rightarrow \pi^{\prime *} E_{i}$ which is an isomorphism off $T^{\prime}$, so by semi-continuity of cohomology there is a non-zero map $\widetilde{E}^{\prime} \rightarrow L_{1} \oplus L_{2}$, where for notational convenience, the pull-back of $L_{i}$ to $\widetilde{X}^{\prime}$ has been denoted by $L_{i}$ rather than $\pi^{\prime *} \pi^{*} L_{i}$. If this map were an isomorphism off $T^{\prime}$ then $\left(\pi_{*}^{\prime} \widetilde{E}^{\prime}\right)^{* *}=\pi^{*}\left(L_{1} \oplus L_{2}\right)$, but this contradicts the fact that the charge on the former bundle is strictly greater than that on $E$. If the map $\widetilde{E}^{\prime} \rightarrow L_{2}$ (say) is generically non-zero, then after taking the maximal normal extension of the kernel of this map, it follows that there is an exact sequence of the form $0 \rightarrow K_{1} \rightarrow \widetilde{E}^{\prime} \rightarrow K_{2} \otimes \mathcal{J} \rightarrow 0$ where $\mathcal{J} \subset \mathcal{O}_{\widetilde{X}^{\prime}}$ is a sheaf of ideals such that $\operatorname{supp}\left(\mathcal{O}_{\widetilde{X}^{\prime}} / \mathcal{J}\right)$ is a finite set, where $K_{1}, K_{2}$ are line bundles on $\widetilde{X}^{\prime}$ satisfying $K_{1} \otimes K_{2}=L_{1} \otimes L_{2} \otimes \mathcal{O}\left(-T^{\prime}\right)$, and there is a non-zero holomorphic map $K_{2} \rightarrow L_{2}$. Since $\left(\pi_{*} \widetilde{E}^{\prime}\right)^{* *}=E^{\prime}$ and 
$\left(\pi_{*}^{\prime} E^{\prime}\right)^{* *}=E=L_{1} \oplus L_{2}$ it follows that $\pi_{*}^{\prime} \pi_{*} \widetilde{E}^{\prime}$ is semi-stable. Then $\omega \cdot L_{2}=\omega \cdot c_{1}\left(\widetilde{E}^{\prime}\right) / 2 \leq \omega \cdot c_{1}\left(K_{2}\right)$, and therefore the map $\left(\pi_{*}^{\prime} \pi_{*} K_{2}\right)^{* *} \rightarrow L_{2}$ must be an isomorphism. Thus $K_{2}=L_{2} \otimes K_{2}^{\prime}$ where $K_{2}^{\prime}$ is trivial off the exceptional divisor in $\widetilde{X}^{\prime}$; moreover, $H^{0}\left(\widetilde{X}^{\prime}, K_{2}^{\prime *}\right) \neq 0$.

Since $\left(\pi_{*} \widetilde{E}^{\prime}\right)^{* *}=E^{\prime}$, the stabilisation construction of $[\mathrm{B} 5, \S 4]$ forces $K_{2}^{\prime}$ to restrict to either $\mathcal{O}(0)$ or $\mathcal{O}(1)$ on each component of $T^{\prime}$ with the latter occurring at most once, and also forces $\operatorname{supp}\left(\mathcal{O}_{\widetilde{X}^{\prime}} / \mathcal{J}\right) \cap T^{\prime}=\emptyset$. Write $K_{2}^{\prime}=: M_{2}^{\prime} \otimes L$ where $L$ is a line bundle on $\widetilde{X}$ which is trivial off $\widetilde{S}$ and $M_{2}^{\prime}$ denotes the line bundle which is trivial off $T^{\prime}$ and has the same restriction to $T^{\prime}$ as $K_{2}^{\prime}$. Then $M_{1}^{\prime}:=\mathcal{O}\left(-T^{\prime}\right) \otimes\left(M_{2}^{\prime}\right)^{*}$ is trivial off $T^{\prime}$, restricts to $\mathcal{O}(0)$ on at most one component of $T^{\prime}$ and to $\mathcal{O}(1)$ on the others. The bundle $\widetilde{E}^{\prime}$ is given by the exact sequence

$$
\tilde{X}^{\prime}: \quad 0 \longrightarrow L_{1} \otimes M_{1}^{\prime} \otimes L^{*} \longrightarrow \widetilde{E}^{\prime} \longrightarrow L_{2} \otimes M_{2}^{\prime} \otimes L \otimes \mathcal{J} \longrightarrow 0 .
$$

The condition that $H^{0}\left(\tilde{X}^{\prime}, K_{2}^{\prime *}\right) \neq 0$ implies $L^{*}$ has a non-zero section, so $\rho_{\beta} \cdot c_{1}(L) \leq 0$. Note that after taking double-duals, $\pi_{*}^{\prime} \pi_{*}(7.1)$ gives the sequence $0 \rightarrow L_{1} \rightarrow\left(\pi_{*}^{\prime} \pi_{*} \widetilde{E}^{\prime}\right)^{* *} \rightarrow L_{2} \otimes \mathcal{J}^{\prime} \rightarrow 0$ for some $\mathcal{J}^{\prime}$ with $\operatorname{supp}\left(\mathcal{O}_{X} / \mathcal{J}^{\prime}\right) \subset \pi \pi^{\prime}\left(\operatorname{supp}\left(\mathcal{O}_{\widetilde{X}^{\prime}} / \mathcal{J}\right)\right) \cup S$, but since $\left(\pi_{*}^{\prime} \pi_{*} \widetilde{E}^{\prime}\right)^{* *}=L_{1} \oplus L_{2}$ the sheaf $\mathcal{J}^{\prime}$ must be isomorphic to $\mathcal{O}_{X}$; hence $\operatorname{supp}\left(\mathcal{O}_{\widetilde{X}^{\prime}} / \mathcal{J}\right) \subset \widetilde{S}$.

The direct image of $(7.1) \otimes \mathcal{O}\left(T^{\prime}\right)$ under $\pi_{*}^{\prime}$ gives the exact sequence

$$
0 \longrightarrow L_{1} \otimes L^{*} \longrightarrow\left(\pi_{*}^{\prime} \widetilde{E}^{\prime}\right)^{* *} \longrightarrow L_{2} \otimes L \otimes \mathcal{J} \longrightarrow 0 .
$$

If $\pi_{*}^{\prime} \widetilde{E}^{\prime}$ is semi-stable then $\omega_{\beta} \cdot c_{1}\left(L_{1} \otimes L^{*}\right) \leq(1 / 2) \omega_{\beta} \cdot c_{1}\left(\pi_{*}^{\prime} \widetilde{E}^{\prime}\right)$, but since $\omega_{\beta} \cdot c_{1}\left(L_{1} \otimes L^{*}\right)=(1 / 2) \omega_{\beta} \cdot c_{1}\left(\pi_{*}^{\prime} \widetilde{E}^{\prime}\right)$ and $\omega_{\beta} \cdot c_{1}\left(L^{*}\right)=\rho_{\beta} \cdot c_{1}\left(L^{*}\right) \geq 0$, it follows $\rho_{\beta} \cdot c_{1}\left(L^{*}\right)=0$. Since $L^{*}$ has a non-zero section, it must therefore be trivial, giving $0 \rightarrow L_{1} \rightarrow\left(\pi_{*}^{\prime} \widetilde{E}^{\prime}\right)^{* *} \rightarrow L_{2} \otimes \mathcal{J} \rightarrow 0$. Since $C\left(\left(\pi_{*}^{\prime} \widetilde{E}^{\prime}\right)^{* *}\right)=C\left(E_{\text {top }}\right)>C(E)$, it follows that $\mathcal{J} \neq \mathcal{O}_{\widetilde{X}}$

Desingularise the sequence $0 \rightarrow L_{1} \rightarrow\left(\pi_{*}^{\prime} \widetilde{E}^{\prime}\right)^{* *} \rightarrow L_{2} \otimes \mathcal{J} \rightarrow 0$ as in [B3,§3] to obtain a blowup $\widehat{X}$ of $\widetilde{X}$ centered at $\operatorname{supp}\left(\mathcal{O}_{\widetilde{X}} / \mathcal{J}\right)$ such that $\hat{\pi}^{*}\left(\left(\pi_{*}^{\prime} \widetilde{E}^{\prime}\right)^{* *}\right)$ is an extension of genuine line bundles, where the line sub-bundle strictly destabilises $\hat{\pi}^{*}\left(\left(\pi_{*}^{\prime} \widetilde{E}^{\prime}\right)^{* *}\right)$ with respect to $\omega_{\beta, \gamma}$ for any suitable $\gamma$. On the other hand,

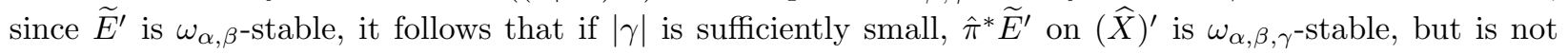
$\omega_{\epsilon \alpha, \beta, \gamma}$-semi-stable for $\epsilon$ sufficiently small.

If each blowup in the sequence $\widetilde{X}_{i}^{\prime}$ is now modified by blowing up in such a way that the new sequence converges to $(\widehat{X})^{\prime}$, then the usual arguments show that (for some subsequence), the pullbacks of $E_{i}^{\prime}$ converge strongly to $\hat{\pi}^{*} \widetilde{E}^{\prime}$ with respect to $\omega_{\alpha, \beta, \gamma}$. Thus, it can be assumed from the outset that in fact $\pi_{*}^{\prime} \widetilde{E}^{\prime}$ is not $\omega_{\beta}$-semi-stable, which implies that $\widetilde{E}^{\prime}$ is not $\omega_{\epsilon \alpha, \beta}$-stable for sufficiently small $\epsilon>0$. In (7.1) therefore, $\rho_{\beta} \cdot L^{*}>0$ else $\pi_{*}^{\prime} \widetilde{E}^{\prime}$ is an extension by torsion-free stable sheaves of the same slope, which is automatically semi-stable.

For each small $\epsilon>0$, let $\widetilde{B}_{\epsilon}^{\prime}$ be the weak limit of the sequence $\pi^{*} E_{i}^{\prime}$ using the metric $\omega_{\epsilon \alpha, \beta}$. Since there are only finitely many different splitting types for $\widetilde{B}_{\epsilon}^{\prime}$, for $\epsilon$ sufficiently small the splitting has topologically constant summands, with degree independent of $\epsilon$. But since $c_{1}\left(\widetilde{B}_{\epsilon}\right)=1$ on each exceptional fibre of $\pi^{\prime}$, it follows that $\widetilde{B}_{\epsilon}$ must be stable for sufficiently small $\epsilon$, and semi-continuity of cohomology implies that this weak limit is in fact independent of such $\epsilon: \widetilde{B}_{\epsilon}^{\prime}=: \widetilde{B}^{\prime}$. Semi-continuity of cohomology also gives a non-zero homomorphism $\widetilde{E}^{\prime} \rightarrow \widetilde{B}^{\prime}$ which must have non-zero kernel at each point (since the two bundles have the same determinants), and by Proposition $1.2,\left(\pi^{\prime} \widetilde{B}^{\prime}\right)^{* *}=: \widetilde{B}$ is $\omega_{\beta}$-semi-stable.

Since $\widetilde{B}^{\prime}$ is $\omega_{\epsilon \alpha, \beta}$-stable for all $\epsilon$ sufficiently small, the composition $L_{1} \otimes M_{1}^{\prime} \otimes L^{*} \rightarrow \widetilde{E}^{\prime} \rightarrow \widetilde{B}^{\prime}$ must be identically zero, so there is a non-zero homomorphism $L_{2} \otimes M_{2}^{\prime} \otimes L \rightarrow \widetilde{B}^{\prime}$. The maximal normal extension of $L_{2} \otimes M_{2}^{\prime} \otimes L \rightarrow \widetilde{B^{\prime}}$ in $\widetilde{B}^{\prime}$ must be of the form $L_{2} \otimes N_{2}^{\prime} \otimes P$ where $N_{2}^{\prime}$ is trivial off $T^{\prime}$ and $P$ is trivial off $\widetilde{S}$, for the fact that $\widetilde{B}^{\prime}$ is $\omega_{\delta \alpha, \beta}$-stable implies (by Proposition 1.2) that $\pi_{*} \pi_{*}^{\prime} \widetilde{B}^{\prime}$ is semi-stable. Thus there is a commutative diagram with exact rows of the form

$$
\begin{aligned}
& 0 \quad \longrightarrow \quad L_{1} \otimes M_{1}^{\prime} \otimes L^{*} \quad \longrightarrow \begin{array}{c}
\widetilde{E}^{\prime} \\
\downarrow
\end{array} \quad \longrightarrow \quad \begin{array}{c}
L_{2} \otimes M_{2}^{\prime} \otimes L \otimes \mathcal{J} \\
\downarrow
\end{array} \quad \longrightarrow \quad 0 \\
& 0 \longleftarrow \mathcal{J}_{1} \otimes L_{1} \otimes N_{1}^{\prime} \otimes P^{*} \longleftarrow \widetilde{B}^{\prime} \longleftarrow \quad L_{2} \otimes N_{2}^{\prime} \otimes P \quad \longleftarrow 0
\end{aligned}
$$

where $N_{1}^{\prime}:=\mathcal{O}\left(-T^{\prime}\right) \otimes\left(N_{2}^{\prime}\right)^{*}$ and $\mathcal{J}_{1}$ is a sheaf of ideals such that $\operatorname{supp}\left(\mathcal{O}_{\widetilde{X}^{\prime}} / \mathcal{J}_{1}\right)$ is a finite set. 
Since there is a non-zero homomorphism $M_{2}^{\prime} \otimes L \rightarrow N_{2}^{\prime} \otimes P$ it follows that $\rho_{\alpha} \cdot c_{1}\left(M_{2}^{\prime}\right) \leq \rho_{\alpha} \cdot c_{1}\left(N_{2}^{\prime}\right)$ and $\rho_{\beta} \cdot c_{1}(L) \leq \rho_{\beta} \cdot c_{1}(P)$. Since $\widetilde{B}^{\prime}$ is $\omega_{\epsilon \alpha, \beta}$-stable for small $\epsilon, \rho_{\beta} \cdot c_{1}(P) \leq 0$. If equality were to hold, then $\omega_{\epsilon \alpha, \beta} \cdot\left(c_{1}\left(\widetilde{B}^{\prime}\right)-2 c_{1}\left(L_{2} \otimes N_{2}^{\prime} \otimes P\right)\right)=\epsilon \rho_{\alpha} \cdot\left(c_{1}\left(\widetilde{B}^{\prime}\right)-2 c_{1}\left(N_{2}^{\prime}\right)\right) \leq \epsilon \rho_{\alpha} \cdot\left(c_{1}\left(\widetilde{B}^{\prime}\right)-2 c_{1}\left(M_{2}^{\prime}\right)\right)<0$, violating stability. Therefore $\rho_{\beta} \cdot c_{1}(P)<0$ implying in particular $C\left(\left(\pi_{*}^{\prime} \widetilde{B}^{\prime}\right)^{* *}\right)>C(E)$.

As in the case of $\widetilde{E}^{\prime}$, there is a non-zero holomorphic map $\widetilde{B}^{\prime} \rightarrow L_{1} \oplus L_{2}$. Since $C\left(\left(\pi_{*}^{\prime} \widetilde{B}^{\prime}\right)^{* *}\right)>C\left(L_{1} \oplus L_{2}\right)$ and $\operatorname{det} \pi_{*}^{\prime} \widetilde{B}^{\prime}=L_{1} \otimes L_{2}$, the map must have non-zero kernel everywhere. If the composition $L_{2} \otimes N_{2}^{\prime} \otimes P \rightarrow$ $\widetilde{B}^{\prime} \rightarrow L_{1} \oplus L_{2}$ were 0 , there would be a non-zero map $L_{1} \otimes N_{1}^{\prime} \otimes P^{*} \rightarrow L_{1} \oplus L_{2}$, which is impossible since $\omega_{\epsilon \alpha, \beta} \cdot c_{1}\left(N_{1}^{\prime} \otimes P^{*}\right)>0$ for small $\epsilon$. If $L_{2} \otimes N_{2}^{\prime} \otimes P \rightarrow \widetilde{B}^{\prime} \rightarrow L_{1}$ were non-zero, then since $L_{1}$ and $L_{2}$ have the same degree, it would follow that $L_{1}=L_{2}$. Thus it can be supposed without loss of generality that $L_{2} \otimes N_{2}^{\prime} \otimes P \rightarrow \widetilde{B^{\prime}} \rightarrow L_{2}$ is non-zero.

Since $\pi_{*}^{\prime} \pi_{*} \widetilde{B}^{\prime}$ is semi-stable, the image of $\widetilde{B^{\prime}} \rightarrow L_{2}$ is of the form $L_{2} \otimes K_{2} \otimes \mathcal{J}_{2}$ for some (new) line bundle $K_{2}$ trivial off the exceptional divisor for which $K_{2}^{*}$ has a non-zero section. Writing $K_{2}=K_{2}^{\prime} \otimes K_{2}^{\prime \prime}$ where $K_{2}^{\prime}$ is trivial off $T^{\prime}$ and $K_{2}^{\prime \prime}$ is trivial off $\widetilde{S}$ and setting $K_{1}^{\prime \prime}:=\left(K_{2}^{\prime}\right)^{*}, K_{1}^{\prime}:=\left(K_{2}^{\prime \prime}\right)^{*} \otimes \mathcal{O}\left(-T^{\prime}\right)$, there is an exact sequence $0 \rightarrow L_{1} \otimes K_{1}^{\prime} \otimes K_{1}^{\prime \prime} \rightarrow \widetilde{B}^{\prime} \rightarrow L_{2} \otimes K_{2}^{\prime} \otimes K_{2}^{\prime \prime} \otimes \mathcal{J}_{2} \rightarrow 0$. Moreover, there are non-zero maps $N_{2}^{\prime} \otimes P \rightarrow K_{2}^{\prime} \otimes K_{2}^{\prime \prime} \otimes \mathcal{J}_{2}$ and $K_{1}^{\prime} \otimes K_{1}^{\prime \prime} \rightarrow N_{1}^{\prime} \otimes P^{*} \otimes \mathcal{J}$, so it follows $\rho_{\alpha} \cdot c_{1}\left(N_{2}^{\prime}\right) \leq$ $\rho_{\alpha} \cdot c_{1}\left(K_{2}^{\prime}\right) \leq 0$ and $\rho_{\beta} \cdot c_{1}(P) \leq \rho_{\beta} \cdot c_{1}\left(K_{2}^{\prime \prime}\right) \leq 0$. Stability of $\widetilde{B}^{\prime}$ with respect to $\omega_{\epsilon \alpha, \beta}$ for all small $\epsilon$ implies $\epsilon \rho_{\alpha} \cdot c_{1}\left(K_{2}^{\prime}\right)+\rho_{\beta} \cdot c_{1}\left(K_{2}^{\prime \prime}\right)>(1 / 2)\left(\epsilon \rho_{\alpha}+\rho_{\beta}\right) \cdot c_{1}\left(\widetilde{B}^{\prime}\right)=-(3 / 2) \epsilon \epsilon_{0}$ for all small $\epsilon>0$. Hence $\rho_{\beta} \cdot c_{1}\left(K_{2}^{\prime \prime}\right)=0$ and since $\left(K_{2}^{\prime \prime}\right)^{*}$ has a non-zero section, it follows that $K_{2}^{\prime \prime}$ is trivial. Furthermore, $K_{2}^{\prime}$ can restrict to $\mathcal{O}(1)$ on at most one component of $T^{\prime}$, restricting trivially to the other components. Since $M_{2}^{\prime}$ also satisfies this condition and there is a non-zero map $M_{2}^{\prime} \rightarrow N_{2}^{\prime}$, on each component of $T^{\prime}$ the latter can restrict only to $\mathcal{O}(1-a)$ for some $a \geq 0$, with $a \geq 1$ on at least 2 components. The existence of the non-zero map $N_{2}^{\prime} \rightarrow K_{2}^{\prime}$ then implies exactly the same behaviour for $N_{2}^{\prime}$; moreover, if $K_{2}^{\prime}$ does restrict to $\mathcal{O}(1)$ on some component of $T^{\prime}$, then the same is true for $N_{2}^{\prime}$ and $M_{2}^{\prime}$ on that component and $K_{2}^{\prime}=N_{2}^{\prime}=M_{2}^{\prime}$.

The following commutative diagram with exact rows and columns summarises the situation for $\widetilde{B}^{\prime}$; in this diagram, the sheaf $Q$ is the pushout:

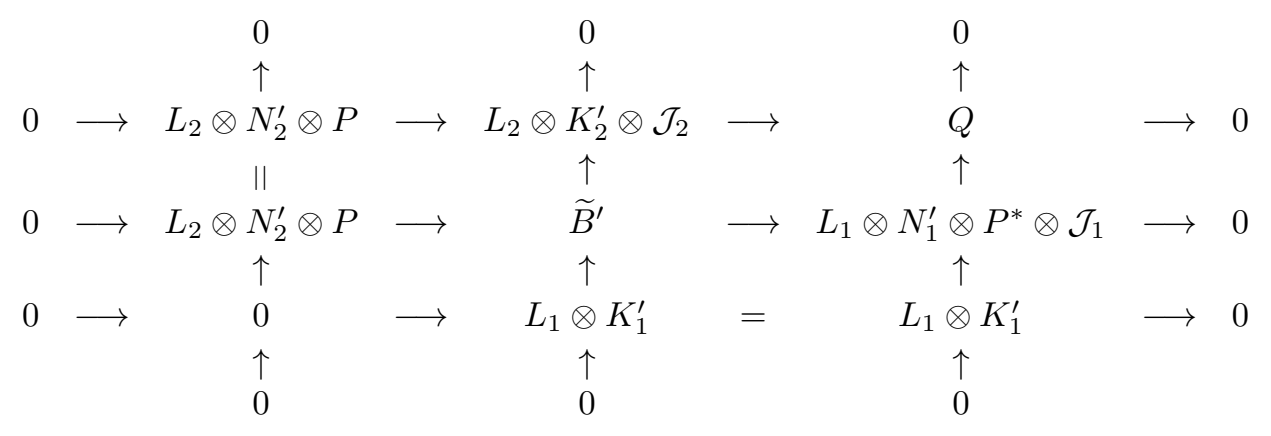

The skyscraper sheaves $\mathcal{O}_{\widetilde{X}^{\prime}} / \mathcal{J}_{1}, \mathcal{O}_{\widetilde{X}^{\prime}} / \mathcal{J}_{2}$ must both be supported in $T^{\prime} \cup \widetilde{S}$, and if $\mathcal{J}_{i}^{\prime}$ is the sheaf of ideals which equals $\mathcal{J}_{i}$ near $\widetilde{S}$ and $\mathcal{O}$ elsewhere, the Riemann-Roch formula gives $\operatorname{dim}\left(\mathcal{O}_{\widetilde{X}^{\prime}} / \mathcal{J}_{2}^{\prime}\right)=\operatorname{dim}\left(\mathcal{O}_{\widetilde{X}^{\prime}} / \mathcal{J}^{\prime}{ }_{1}\right)-$ $c_{1}(P)^{2}>0 ;$ thus, $\operatorname{supp}\left(\mathcal{O}_{\widetilde{X}^{\prime}} / \mathcal{J}_{2}\right) \cap \widetilde{S} \neq \emptyset$.

As was done for $\widetilde{E}^{\prime}$ above, desingularise the sequence $0 \rightarrow L_{1} \otimes K_{1}^{\prime} \rightarrow \widetilde{B}^{\prime} \rightarrow L_{2} \otimes K_{2}^{\prime} \otimes \mathcal{J}_{2} \rightarrow 0$ as in [B3] by blowing up $\widetilde{X}^{\prime}$ along the points of $\operatorname{supp}\left(\mathcal{O}_{\widetilde{X}^{\prime}} / \mathcal{J}_{2}\right) \cap \widetilde{S}$ to express the pull-back of $\widetilde{B}^{\prime}$ to this blowup $\left(\widetilde{X}_{1}^{\prime} \stackrel{\pi_{1}}{\rightarrow} \widetilde{X}^{\prime}\right.$ say) as an extension by genuine line bundles (except perhaps near $\left.T^{\prime}\right)$. For any suitable $\gamma$, the new line sub-bundle strictly destabilises $\pi_{*}^{\prime} \widetilde{B}^{\prime}$ with respect to $\omega_{\beta, \epsilon \gamma}$ for all $\epsilon>0$, which implies that $\pi_{1}^{*} \widetilde{B}^{\prime}$ is $\omega_{\epsilon \alpha, \beta, \gamma}$-stable for some small $\epsilon>0$, but is not $\omega_{\epsilon^{\prime} \alpha, \beta, \gamma}$-stable for some $\epsilon^{\prime}<\epsilon$.

Fix $\epsilon>0$ and $\gamma$ with $|\gamma|$ and $\epsilon$ chosen sufficiently small that $\omega_{\alpha, \beta, \gamma}$ is suitable and so that for $c \epsilon$ $H^{2}\left(\widetilde{X}_{1}^{\prime}, \mathbb{Z}\right)$, the constraints

$$
\omega \cdot c=0, \quad\left|\rho_{\beta} \cdot c\right| \leq 2(\epsilon|\alpha|+|\gamma|) \sqrt{C\left(E_{\mathrm{top}}\right)+1}, \quad-c \cdot c \leq 4\left(C\left(E_{\mathrm{top}}\right)+1\right)
$$

imply $\rho_{\beta} \cdot c=0$. Now repeat the same construction as before to obtain weak limits on $\tilde{X}_{1}^{\prime}$ : using the metric $\omega_{\alpha, \beta, \gamma}$, the pull-backs of $E_{i}^{\prime}$ must converge strongly to $\pi_{1}^{*} \widetilde{E}^{\prime}$; with respect to $\omega_{\epsilon \alpha, \beta, \gamma}$ for any sufficiently small $\epsilon$ they converge to some new stable bundle $\widetilde{B}_{1}^{\prime}$.

There are two cases to consider; namely, $\pi_{1 *} \widetilde{B}_{1}^{\prime}$ on $\widetilde{X}^{\prime}$ is or is not $\omega_{\epsilon \alpha, \beta}$-semi-stable. In the former case, since $\widetilde{B}^{\prime}$ is $\omega_{\epsilon \alpha, \beta}$-stable and there is a non-zero holomorphic map $\left(\pi_{1 *} \widetilde{B}_{1}^{\prime}\right)^{* *} \rightarrow \widetilde{B}^{\prime}$, it follows $\left(\pi_{1 *} \widetilde{B}_{1}^{\prime}\right)^{* *}=\widetilde{B}^{\prime}$. 
Since $\widetilde{B}_{1}^{\prime}$ is $\omega_{\epsilon \alpha, \beta, \gamma}$-stable but $\pi_{1}^{*} \widetilde{B}^{\prime}$ is not, it follows in this case that $\widetilde{B}_{1}^{\prime}$ must be non-trivial on the exceptional fibres of $\pi_{1}$ and therefore $C\left(\widetilde{B}_{1}^{\prime}\right)>C\left(\widetilde{B}^{\prime}\right)$ ([B5, Prop. 2.9]).

Assuming as usual that the amount of charge bubbled by the pull-backs of $E_{i}$ to $\widetilde{X}_{1}^{\prime}$ is the same as the original, the same process as above can be repeated until such time as the direct image of $\widetilde{B}_{i}^{\prime}$ onto $\widetilde{X}_{i-1}^{\prime}$ is not semi-stable and the situation is that of the second possibility.

If $\pi_{1 *} \widetilde{B}_{1}^{\prime}$ is not $\omega_{\epsilon \alpha, \beta}$-semi-stable, then for some $\delta \in(0,1)$ there is a line bundle $R$ on $\widetilde{X}_{1}^{\prime}$ with a sheaf inclusion $R \rightarrow \widetilde{B}_{1}^{\prime}$ with torsion-free quotient such that $\omega_{\epsilon \alpha, \beta, \delta \gamma} \cdot\left(c_{1}\left(\widetilde{B}_{1}^{\prime}\right)-2 c_{1}(R)\right)=0$. The same arguments as above lead to the conclusion that $R$ must be of the form $L_{1}$ or $L_{2}$ tensored with a line bundle trivial off the exceptional divisor, and that $\rho_{\epsilon \alpha, \beta, \delta \gamma} \cdot\left(c_{1}\left(\widetilde{B}_{1}^{\prime}\right)-2 c_{1}(R)\right)=0$. Stability at $\delta=1$ implies $\rho_{\gamma} \cdot\left(c_{1}\left(\widetilde{B}_{1}^{\prime}\right)-2 c_{1}(R)\right)>0$, so $\rho_{\epsilon \alpha, \beta} \cdot\left(c_{1}\left(\widetilde{B}_{1}^{\prime}\right)-2 c_{1}(R)\right)<0$. Stability with respect to $\omega_{\epsilon \alpha, \beta, \gamma}$ for all $\epsilon>0$ implies $\rho_{\beta, \gamma} \cdot\left(c_{1}\left(\widetilde{B}_{1}^{\prime}\right)-2 c_{1}(R)\right) \geq 0$. Since $\left|\rho_{\beta} \cdot\left(c_{1}\left(\widetilde{B}_{1}^{\prime}\right)-2 c_{1}(R)\right)\right| \leq(\epsilon|\alpha|+|\gamma|)|| c_{1}\left(\widetilde{B}_{1}^{\prime}\right)-2 c_{1}(R) \|$, by (7.4) and choice of $\epsilon$ and $|\gamma|$ the bundle $R$ must satisfy $\rho_{\beta} \cdot c_{1}(R)=0$, so to summarise,

$$
\begin{array}{rrr}
\omega \cdot\left(c_{1}\left(\widetilde{B}_{1}^{\prime}\right)-2 c_{1}(R)\right)=0, & \rho_{\alpha} \cdot\left(c_{1}\left(\widetilde{B}_{1}^{\prime}\right)-2 c_{1}(R)\right)<0 \\
\rho_{\beta} \cdot\left(c_{1}\left(\widetilde{B}_{1}^{\prime}\right)-2 c_{1}(R)\right)=0, & \rho_{\gamma} \cdot\left(c_{1}\left(\widetilde{B}_{1}^{\prime}\right)-2 c_{1}(R)\right)>0 .
\end{array}
$$

As in the case of $\widetilde{B}^{\prime}$, there is a non-zero homomorphism $\widetilde{B}_{1}^{\prime} \rightarrow L_{1} \oplus L_{2}$. The composition $R \rightarrow \widetilde{B}_{1}^{\prime} \rightarrow$ $L_{1} \oplus L_{2}$ cannot be identically zero, else there is a non-zero map $R^{*} \otimes \operatorname{det}\left(\widetilde{B}_{1}^{\prime}\right) \rightarrow L_{1} \oplus L_{2}$ which contradicts the fact that $\omega_{\epsilon \alpha, \beta, \gamma} \cdot\left(c_{1}\left(L_{i}\right)-c_{1}\left(R^{*} \otimes \operatorname{det}\left(\widetilde{B}_{1}^{\prime}\right)\right)\right)<0$ for $\epsilon$ sufficiently small. Hence on each component of $T^{\prime}, R$ must restrict to $\mathcal{O}(a)$ for $a \geq 0$, and since $\rho_{\alpha} \cdot c_{1}(R)>(1 / 2) \rho_{\alpha} \cdot c_{1}\left(\widetilde{B}_{1}^{\prime}\right)$ it follows that $R$ can only restrict to $\mathcal{O}(1)$ or $\mathcal{O}(0)$ with the former occurring at most once.

Semi-continuity of cohomology gives a non-zero holomorphic map $\left(\pi_{1 *} \widetilde{B}_{1}^{\prime}\right)^{* *} \rightarrow \widetilde{B}^{\prime}$, but since the former is unstable and the latter is stable, this cannot be an isomorphism. The induced map $\left(\pi_{1 *} R\right)^{* *} \rightarrow \widetilde{B}^{\prime}$ must be identically 0 since $\omega_{\epsilon \alpha, \beta} \cdot\left(c_{1}\left(\widetilde{B}_{1}\right)-2 c_{1}(R)\right)<0$, so there is an induced map $\left(\pi_{1 *} R^{*} \otimes \operatorname{det}\left(\widetilde{B}_{1}^{\prime}\right)\right)^{* *} \rightarrow \widetilde{B}^{\prime}$. If the composition of this map with $\widetilde{B}^{\prime} \rightarrow L_{1} \otimes N_{1}^{\prime} \otimes P^{*} \otimes \mathcal{J}_{1}$ were identically 0 , there would be an induced non-zero map $\left(\pi_{1 *} R^{*} \otimes \operatorname{det}\left(\widetilde{B}_{1}^{\prime}\right)\right)^{* *} \rightarrow L_{2} \otimes N_{2}^{\prime} \otimes P$, and hence a non-zero section of $R \otimes L_{1}^{*} \otimes N_{1}^{\prime *} \otimes P$. This however is not possible since $\omega_{\epsilon \alpha, \beta} \cdot c_{1}\left(R \otimes L_{1}^{*} \otimes N_{1}^{\prime *} \otimes P\right)=\epsilon \rho_{\alpha} \cdot c_{1}\left(R \otimes N_{1}^{\prime *}\right)+\rho_{\beta} \cdot c_{1}(P)$ is negative for sufficiently small $\epsilon$. Thus $R$ has the form $L_{2} \otimes S_{2}^{\prime} \otimes \hat{L}$ for some $S_{2}^{\prime}$ on $X^{\prime}$ trivial off $T^{\prime}$ and some $\hat{L}$ on $\tilde{X}_{1}^{\prime}$ which is trivial off the exceptional fibres of $\pi_{1}$, using here the facts that $\rho_{\beta} \cdot c_{1}(R)=0$ and $\beta$ is assumed generic. Moreover $S_{2}^{\prime}$ restricts to $\mathcal{O}(1)$ on at most one component of $T^{\prime}$ and to $\mathcal{O}(0)$ on the others, and there is a non-zero holomorphic map $N_{2}^{\prime} \rightarrow S_{2}^{\prime}$ (so as before, if $S_{2}^{\prime}$ does restrict to $\mathcal{O}(1)$ on some component of $T^{\prime}$ then the same is true of $N_{2}^{\prime}$ and $M_{2}^{\prime}$ ), and $\rho_{\gamma} \cdot \hat{L}<0$.

By semi-continuity of cohomology once more, there is a non-zero holomorphic map $\pi_{1}^{*} \widetilde{E}^{\prime} \rightarrow \widetilde{B}_{1}^{\prime}$. Pull back the sequence (7.1) to $\widetilde{X}_{1}^{\prime}$ and quotient out $\pi_{1}^{*}\left(L_{2} \otimes M_{2}^{\prime} \otimes L \otimes \mathcal{J}\right)$ by its torsion subsheaf to obtain a new sequence $0 \rightarrow L_{1} \otimes M_{1}^{\prime} \otimes L^{*} \otimes \hat{M}^{*} \rightarrow \widetilde{E}^{\prime} \rightarrow L_{2} \otimes M_{2}^{\prime} \otimes L \otimes \hat{M} \otimes \hat{\mathcal{J}} \rightarrow 0$ for some line bundle $\hat{M}$ trivial off the exceptional fibre of $\pi_{1}$ satisfying $\rho_{\gamma} \cdot c_{1}(\hat{M}) \leq 0$ and some sheaf of ideals $\hat{\mathcal{J}} \subset \mathcal{O}_{\widetilde{X}_{1}^{\prime}}$ such that $\operatorname{supp}\left(\mathcal{O}_{\widetilde{X}_{1}^{\prime}} / \hat{\mathcal{J}}\right)$ is a finite set. Setting $S_{1}^{\prime}:=S_{2}^{\prime *} \otimes \mathcal{O}\left(-T^{\prime}\right)$, there is a diagram

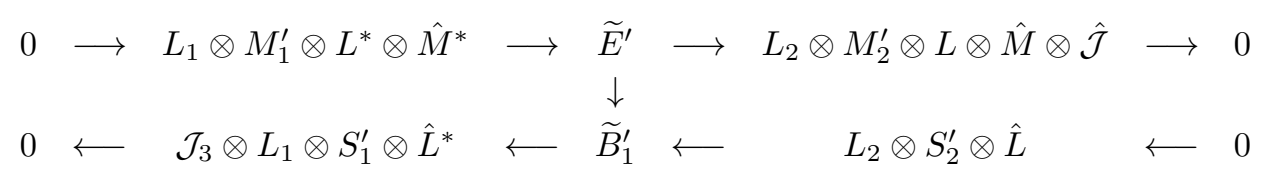

where $\mathcal{J}_{3}$ is another sheaf of ideals of the same type as $\hat{\mathcal{J}}$. The induced map $L_{1} \otimes M_{1}^{\prime} \otimes L^{*} \otimes \hat{M}^{*} \rightarrow \widetilde{B}_{1}^{\prime}$ must be identically 0 since $\omega_{\epsilon \alpha, \beta, \gamma} \cdot\left(c_{1}\left(\widetilde{B}_{1}^{\prime}\right)-2 c_{1}\left(L_{1} \otimes M_{1}^{\prime} \otimes L^{*} \otimes \hat{M}^{*}\right)\right) \leq \omega_{\epsilon \alpha, \beta, \gamma} \cdot\left(c_{1}\left(\widetilde{E}^{\prime}\right)-2 c_{1}\left(L_{1} \otimes M_{1}^{\prime} \otimes L^{*}\right)<0\right.$ for small $\epsilon>0$. Therefore there is an induced map $L_{2} \otimes M_{2}^{\prime} \otimes L \otimes \hat{M} \rightarrow \widetilde{B}_{1}^{\prime}$, and the composition of this map with the projection $\widetilde{B}_{1}^{\prime} \rightarrow L_{1} \otimes S_{1}^{\prime} \otimes \hat{L}^{*}$ must also vanish identically since $M_{2}^{\prime}$ must restrict to $\mathcal{O}(0)$ on some component of $T^{\prime}$ on which $S_{1}^{\prime}$ restricts to $\mathcal{O}(1)$ (implying that $\operatorname{deg}\left(L_{2} \otimes M_{2}^{\prime} \otimes L \otimes \hat{M}\right)>\operatorname{deg}\left(L_{1} \otimes S_{1}^{\prime} \otimes \hat{L}^{*}\right)$ with respect to some appropriate metric on $\widetilde{X}_{1}^{\prime}$ ). It follows that there is a non-zero holomorphic map $L_{2} \otimes M_{2}^{\prime} \otimes L \otimes \hat{M} \rightarrow L_{2} \otimes S_{2}^{\prime} \otimes \hat{L}$, so $\hat{L} \otimes \hat{M}^{*}$ has a non-zero section. Thus $\rho_{\gamma} \cdot \hat{M} \leq \rho_{\gamma} \cdot \hat{L}<0$, implying that $\hat{M}$ cannot be trivial on all the exceptional fibres of $\pi_{1}$.

Since $C\left(\widetilde{E}^{\prime}\right) \geq-(1 / 4)\left[\left(c_{1}\left(L_{1}\right)-c_{1}\left(L_{2}\right)\right)^{2}+2 c_{1}(L)^{2}+2 c_{1}(\hat{M})^{2}\right]$, it follows that after repeating this construction (of $\widetilde{B}_{1}$ ) sufficiently often, this process must eventually terminate and at that point, the pullbacks of the bundles $E_{i}$ must have a subsequence which bubbles a strictly smaller amount of charge than 
$C\left(E_{\text {top }}\right)-C(E)$. Applying the inductive hypothesis then yields a sequence of blowups for which the pull-backs of $E_{i}$ have a strongly convergent subsequence.

A count of the maximum number of blowups required to obtain a strongly convergent subsequence yields the crude bound $\left(C\left(E_{\mathrm{top}}\right)-C(E)\right)^{2}+1$. This estimate can be improved using the following argument, which is applicable for arbitrary rank $r$.

Suppose that for some sequence of blowups $\widetilde{X}_{i}$ converging to $\widetilde{X}, \pi_{i}^{*} E_{i}$ converges strongly to a stable bundle $\widetilde{E}$ with respect to $\omega_{\alpha}$. Let $\widetilde{S}$ be the exceptional divisor in $\widetilde{X}$ and pick a set of $r^{2}+1$ points $T \subset X$ disjoint from a neighbourhood of $\operatorname{supp}\left(\rho_{\alpha}\right)$. Let $E_{0}:=\left(\pi_{*} \widetilde{E}\right)^{* *}$ and stabilise $E_{0}$ to a bundle $E_{0}^{\prime}$ on a blowup $X^{\prime}$ of $X$ centered at $T$. Since $\pi^{*} E_{0}=\widetilde{E}$ near $T$, there is a corresponding "stabilisation" $\widetilde{E}^{\prime}$ of $\widetilde{E}$, and by construction, $\widetilde{E}^{\prime}$ is stable with respect to $\omega_{\epsilon \alpha, \delta \beta}$ for all $\epsilon, \delta \in(0,1]$. The convergence of $\pi_{i}^{*} E_{i}$ to $\widetilde{E}$ implies that there is a corresponding sequence of stabilisations $E_{i}^{\prime}$ of $E_{i}$ such that $\pi_{i}^{*} E_{i}^{\prime}$ converges to $\widetilde{E}^{\prime}$ with respect to $\omega_{\epsilon \alpha, \delta \beta}$.

After passing to a subsequence if necessary, there is a finite set $S^{\prime} \subset X^{\prime}$ such that $\left\{E_{i}^{\prime}\right\}$ converges weakly off $S^{\prime}$ to a quasi-stable bundle with respect to $\omega_{\beta}$. Stability of $E_{0}^{\prime}$ and semi-continuity of cohomology imply that this bundle is isomorphic to $E_{0}^{\prime}$. Applying Lemma 2.1 on $\widetilde{X}^{\prime} \backslash \operatorname{supp}\left(\rho_{\alpha}\right)$ and using the strong convergence of the sequence $\pi^{*} E_{i}^{\prime}$, it follows that $S^{\prime} \subset X^{\prime} \backslash \pi\left(\operatorname{supp}\left(\rho_{\alpha}\right)\right)$; that is, there is no bubbling of curvature near $\pi^{\prime-1}(T)$ for the sequence $\left\{E_{i}^{\prime}\right\}$. Furthermore, the amount of charge bubbled by this sequence is at most $C\left(E_{\text {top }}\right)$.

Now apply Theorem 1.3 to obtain a sequence $\left\{\hat{X}_{i}^{\prime}\right\}$ of blowups of $X^{\prime}$ consisting of at most $2 C\left(E_{\mathrm{top}}\right)-1$ blowups converging to a blowup $\hat{X}^{\prime}$ of $X^{\prime}$ for which, after passing to a subsequence if necessary, $\hat{\pi}_{i}^{*} E_{i}^{\prime}$ converges to a stable bundle $\hat{E}^{\prime}$ with respect to $\omega_{\beta, \gamma}$, where $|\gamma|$ is taken to be so small that the pull-back from $X^{\prime}$ of a bundle of charge $\leq C\left(\widetilde{E}^{\prime}\right)$ which is $\omega_{\beta}$-stable is $\omega_{\beta, \gamma}$-stable. By Proposition $1.2, \gamma$ can be replaced by $\delta \gamma$ for any $\delta \in(0,1)$ to obtain the same limit $\widetilde{E}^{\prime}$. The exceptional divisor of $\hat{X}^{\prime} \rightarrow X^{\prime}$ is $\hat{\pi}^{-1}\left(S^{\prime}\right)$ and $\left(\hat{\pi}_{*} \hat{E}^{\prime}\right)^{* *}=E_{0}^{\prime}$.

Semi-continuity of cohomology implies that on a sequence of joint blowups of $\hat{X}_{i}^{\prime}$ and $\widetilde{X}_{i}$, the pull-backs of $E_{i}^{\prime}$ converge to the pull-back of $\widetilde{E}^{\prime}$ with respect to $\omega_{\alpha, \beta, \delta \gamma}$ if $\delta>0$ is small enough, and to the pull-back of $\hat{E}^{\prime}$ with respect to $\omega_{\epsilon \alpha, \beta, \gamma}$ for $\epsilon>0$ sufficiently small. Semi-continuity of cohomology gives a non-zero holomorphic map between these two pull-backs which pushes down to a non-zero map between the double duals of the direct images on $X^{\prime}$. But since both such push-downs are isomorphic to the stable bundle $E_{0}^{\prime}$, it follows that the map is an isomorphism off the exceptional divisor, and since the two bundles have the same determinant, it is an isomorphism everywhere.

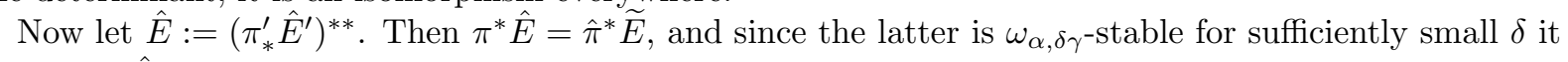

follows that $\hat{E}$ must be $\omega_{\delta \gamma}$-stable. Semi-continuity of cohomology now implies that $\left\{\hat{\pi}_{i}^{*} E_{i}\right\}$ has a subsequence strongly convergent to $\hat{E}$ with respect to $\omega_{\delta \gamma}$. This completes the proof of Theorem 1.4.

\section{REFERENCES}

[AHS] M. F. Atiyah, N. J. Hitchin and I. M. Singer, Self-duality in four dimensional Riemannian geometry, Proc. Roy. Soc. Lond. Ser. A 362 (1978) 425-461.

[BPV] W. Barth, C. Peters and A. Van de Ven, Compact Complex Surfaces, (Springer, Berlin, Heidelberg, New York, 1984).

[B1] N. P. Buchdahl, "Instantons on $\mathbb{C P}_{2}$ ", J. Differ. Geom. 24 (1986) 19-52.

[B2] N. P. Buchdahl, "Stable 2-bundles on Hirzebruch surfaces", Math. Z. 194 (1987) 143-152.

[B3] N. P. Buchdahl, "Hermitian-Einstein connections and stable vector bundles over compact complex surfaces", Math. Ann. 280 (1988) 625-648.

[B4] N. P. Buchdahl, "Instantons on $n \mathbb{C P}_{2}$ ", J. Differ. Geom 37 (1993) 669-687.

[B5] N. P. Buchdahl, "Blowups and gauge fields". Preprint (1995).

[D1] S. K. Donaldson, "Instantons and geometric invariant theory", Commun. Math. Phys. 93 (1984) 453460. 
[D2] S. K. Donaldson, "Anti-self-dual Yang-Mills connections over complex algebraic varieties and stable vector bundles", Proc. Lond. Math. Soc. 50 (1985) 1-26.

[D3] S. K. Donaldson, "Connections, cohomology and the intersection forms of 4-manifolds", J. Differ. Geom. 24 (1986) 275-341.

[D4] S. K. Donaldson, "Irrationality and the h-cobordism conjecture ", J. Differ. Geom. 26 (1987) 141-168.

[D5] S. K. Donaldson, "Polynomial invariants for smooth four manifolds", Topology 29 (1990) 257-315.

[FU] D. S. Freed and K. K. Uhlenbeck, Instantons and Four-Manifolds, MSRI Publications Vol 1. (Springer, New York, Berlin, Heidelberg, Tokyo, 1984).

[FM1] R. Friedman and J. W. Morgan, "On the diffeomorphism types of certain algebraic surfaces I", $J$. Differ. Geom. 27 (1988) 297-369.

[FM2] R. Friedman and J. W. Morgan, "On the diffeomorphism types of certain algebraic surfaces II", J. Differ. Geom. 27 (1988) 371-398.

[Gau] P. Gauduchon, "Le théorème de l'excentricité nulle", C. R. Acad. Sci. Paris 285 (1977) 387-390.

[Gie] D. Gieseker, "On the moduli of vector bundles on an algebraic surface", Ann. Math. 106 (1977) 45-60.

[GT] D. Gilbarg and N. S. Trudinger, Elliptic partial differential equations of second order. 2nd ed. (Springer: Berlin Heidelberg New York, 1983).

[Ki] A. D. King, "Instantons and holomorphic bundles on the blown-up plane", D. Phil. Thesis Oxford (1989).

[Kob] S. Kobayashi, "Curvature and stability of vector bundles", Proc. Japan Acad. Ser. A Math. Sci. 58 (1982) 158-162.

[Kot] D. Kotschick, "On manifolds homeomorphic to $\mathbb{C P}^{2} \# 8 \overline{\mathbb{C P}}^{2}$ ", Invent. Math. 95 (1989) 591-600.

[L] M. Lübke, "Stability of Einstein-Hermitian vector bundles", Manuscr. Math. 42 (1983) 245-257.

[LY] J. Li and S. -T. Yau, "Hermitian-Yang-Mills connection on non-Kähler manifolds". In: Mathematical Aspects of String Theory, ed S. -T. Yau (World Scientific: Singapore, 1987).

[Ma] M. Maruyama, "On a compactification of a moduli space of stable bundles on a rational surface". In Algebraic Geometry and Commutative Algebra, Kinokuniya, Tokyo 1988. 233-260.

[Mor] J. W. Morgan, "Comparison of the Donaldson polynomial invariants with their algebro-geometric analogues", Topology 32 (1993) 449-488.

[OSS] C. Okonek, M. Schneider and H. Spindler, Vector bundles on complex projective spaces, (Birkhäuser, Boston, Basel, Stuttgart, 1980).

[OV] C. Okonek and A. Van de Ven, "Stable bundles and differentiable structures on certain algebraic surfaces", Invent. Math. 86 (1986) 357-370.

[S] S. Sedlacek, "A direct method for minimizing the Yang-Mills functional", Commun. Math. Phys. 86 (1982) 515-528.

[U1] K. K. Uhlenbeck, "Connections with $L^{p}$ bounds on curvature", Commun. Math. Phys. 83 (1982) 31-42.

[U2] K. K. Uhlenbeck, "Removable singularities in Yang-Mills fields", Commun. Math. Phys. 83 (1982) $11-30$.

[UY] K. K. Uhlenbeck and S. -T. Yau, "On the existence of Hermitian-Yang-Mills connections in stable vector bundles", Commun. Pure App. Math. 39 (1986) 257-293. 